quatrième série-tome 41 fascicule 1 janvier-février 2008

$$
\begin{aligned}
& \text { ANNALES } \\
& \text { SCIENTIFIQUES } \\
& \text { de } \\
& \text { L'ECOLE } \\
& \text { NORMALE } \\
& \text { SUPÉRIEURE }
\end{aligned}
$$

\title{
Erwan LANNEAU
}

Connected components of the strata of the moduli spaces of quadratic differentials 


\title{
CONNECTED COMPONENTS OF THE STRATA OF THE MODULI SPACES OF QUADRATIC DIFFERENTIALS
}

\author{
BY ERWAN LANNEAU
}

\begin{abstract}
In two fundamental classical papers, Masur [14] and Veech [21] have independently proved that the Teichmüller geodesic flow acts ergodically on each connected component of each stratum of the moduli space of quadratic differentials. It is therefore interesting to have a classification of the ergodic components. Veech has proved that these strata are not necessarily connected. In a recent work [8], Kontsevich and Zorich have completely classified the components in the particular case where the quadratic differentials are given by the global square of Abelian differentials.

Here we are interested in the complementary case. In a previous paper [11], we have described some particular components, namely the hyperelliptic connected components and showed that some strata are non-connected. In this paper, we give the general classification theorem: up to four exceptional cases in low genera, the strata of meromorphic quadratic differentials are either connected, or have exactly two connected components where one component is hyperelliptic, the other not. This result was announced in the paper [11].

Our proof is based on a new approach of the so-called Jenkins-Strebel differential. We will present and use the notion of generalized permutations.
\end{abstract}


RÉsumé. - Dans des travaux maintenant classiques, Masur [14] et Veech [21] ont démontré indépendamment que le flot géodésique de Teichmüller est ergodique sur chaque composante connexe de chaque strate de l'espace des modules des différentielles quadratiques. Il devient dès lors intéressant d'avoir une description de ces composantes ergodiques. Veech a montré que ces strates ne sont pas nécessairement connexes. Dans un article récent, Kontsevich et Zorich [8] donnent une description complète des composantes dans le cas particulier où les différentielles quadratiques sont données par le carré de différentielles abéliennes.

Dans cet article, nous considérons le cas complémentaire. Dans un précédent article [11], nous montrions que les strates ne sont pas forcément connexes. Nous donnions une série de strates non-connexes possédant des composantes connexes hyperelliptiques. Dans cet article, nous démontrons le théorème général annoncé dans [11] : excepté quatre cas particuliers en petits genres, les strates de l'espace des modules des différentielles quadratiques ont au plus deux composantes connexes, les cas de non-connexité étant décrits exactement par [11] : une composante est hyperelliptique, l'autre non.

Notre preuve repose principalement sur une nouvelle approche des différentielles quadratiques de type Jenkins-Strebel, à savoir la notion de permutations généralisées.

\section{Introduction}

The moduli space of a genus $g$ compact connected Riemann surface $\mathcal{S}$ endowed with an integrable meromorphic quadratic differential $q$ is a disjoint union $\mathcal{H}_{g} \sqcup \mathcal{Q}_{g}$, where the isomorphism class of $(\mathcal{S}, q)$ belongs to $\mathcal{H}_{g}$ if and only if $q$ is the (global) square of a holomorphic Abelian differential. It can be identified with the cotangent bundle of the moduli space $\mathcal{M}_{g}$ of compact connected smooth complex curves (see e.g. [5]). It carries a natural flow, called the Teichmüller geodesic flow (see [14, 21]). It has a natural stratification, whose strata are denoted by $\mathcal{H}\left(k_{1}, \cdots, k_{n}\right)=\mathcal{H}_{g}\left(k_{1}, \cdots, k_{n}\right)$ contained in $\mathcal{H}_{g}$ and $\mathcal{Q}\left(k_{1}, \cdots, k_{n}\right)=$ $\mathcal{Q}_{g}\left(k_{1}, \cdots, k_{n}\right)$ contained in $\mathcal{Q}_{g}$, where $k_{1}, \ldots, k_{n}$ is the (unordered) list of multiplicities of the zeroes and poles of the quadratic differentials. It is well known that the flow preserves this stratification and that each stratum carries a complex algebraic orbifold structure of complex dimension $2 g+n-\varepsilon$ (here $\varepsilon=1$ or 2 depending respectively of the strata of Abelian differential or quadratic differentials). Masur and Smillie [15] proved that all of these strata (corresponding to the multiplicities satisfying the Gauss-Bonnet condition), except four particular cases in low genera, are non-empty.

The aim of this paper is motivated by a fundamental theorem, independently proved by Masur and Veech [14], [21], which asserts that the Teichmüller geodesic flow acts ergodically on each connected component of each stratum (with respect to a finite measure equivalent to the Lebesgue measure).

Kontsevich and Zorich [8] have recently described the set of connected components for the strata of $\mathcal{H}_{g}$. In [11], using a construction developed in [8] (hyperelliptic components), we showed that some strata in $\mathcal{Q}_{g}$ are non-connected. More precisely, we presented three series of one discrete-parameter strata which are non connected; those strata have a connected component consisting of hyperelliptic curves equipped with a "hyperelliptic differential". This component is called a hyperelliptic component.

$4^{\text {e }}$ SÉRIE - TOME $41-2008-\mathrm{N}^{\mathrm{o}} 1$ 
In this paper, we describe the set of connected components of any stratum of $\mathcal{Q}_{g}$. The general case stabilizes at genus 5 and corresponds to Theorem 1.1. Theorem 1.2 gives the remaining cases.

Theorem 1.1. - Let us fix $g \geq 5$. Each stratum of the moduli space $\mathcal{Q}_{g}$ having a hyperelliptic connected component has exactly two connected components: one is hyperelliptic - the other not; the detailed list is given in [11] (see also below).

Any other stratum of the moduli space $\mathcal{Q}_{g}$ of quadratic differentials is non empty and connected.

In small genera, there are some exceptional cases coming from the geometry of genus one and genus two surfaces (respectively elliptic and hyperelliptic curves). There are also 4 mysterious cases which appear.

THEOREM 1.2. - Let us fix $g \leq 4$. The connected components of the strata of the moduli space $\mathcal{Q}_{g}$ fall in the following description:

- In genera 0 and 1 , except two strata that are empty, any stratum is non empty and connected.

- In genus 2 , there are two empty strata. There are also two non-connected strata. For these two, one component is hyperelliptic, the other not. Any other stratum of $\mathcal{Q}_{2}$ is non empty connected.

- In genera 3 and 4 , each stratum with a hyperelliptic connected component has exactly two connected components: one is hyperelliptic, the other not.

- There are 4 sporadic strata in genus 3 and 4 which are non-connected and which do not possess a hyperelliptic component.

- Any other stratum of $\mathcal{Q}_{3}$ and $\mathcal{Q}_{4}$ is non empty and connected.

\subsection{Precise formulation of the statements}

In order to establish notations and to give a precise statement, we review basic notions concerning moduli spaces, Abelian differentials and quadratic differentials. There is an abundant literature on this subject; for more details and proofs see for instance [2], [3], [4], [5], [9], [8], [14], [19], [20], [21], [23], [22],. . . For a nice survey see [16] or [24].

1.1.1. Background. - For $g \geq 1$, we define the moduli space of Abelian differentials $\mathcal{H}_{g}$ as the moduli space of pairs $(\mathcal{S}, \omega)$ where $\mathcal{S}$ is a genus $g$ (closed connected) Riemann surface and $\omega \in \Omega(\mathcal{S})$ a non-zero holomorphic 1 -form defined on $\mathcal{S}$. The term moduli space means that we identify the points $(\mathcal{S}, \omega)$ and $\left(\mathcal{S}^{\prime}, \omega^{\prime}\right)$ if there exists an analytic isomorphism $f: \mathcal{S} \rightarrow \mathcal{S}^{\prime}$ such that $f^{*} \omega^{\prime}=\omega$.

For $g \geq 0$, we also define the moduli space of quadratic differentials $\mathcal{Q}_{g}$ which are not the global square of Abelian differentials as the moduli space of pairs $(\mathcal{S}, q)$ where $\mathcal{S}$ is a genus $g$ Riemann surface and $q$ a non-zero meromorphic quadratic differential defined on $\mathcal{S}$ such that $q$ is not the global square of any Abelian differential. In addition, we assume that $q$ has at most simple poles, if any. This last condition guaranties that the area of $\mathcal{S}$ in terms of the metric determined by $q$ is finite:

$$
\int_{\mathcal{S}}|q|<\infty
$$


We will denote by $\mathcal{H}\left(k_{1}, \ldots, k_{n}\right)$ the subset of $\mathcal{H}_{g}$ consisting of (classes of) pairs $(\mathcal{S}, \omega)$ such that $\omega$ possesses exactly $n$ zeroes on $\mathcal{S}$ with multiplicities $\left(k_{1}, \ldots, k_{n}\right)$. We will also denote by $\mathcal{Q}\left(k_{1}, \ldots, k_{n}\right)$ the subset of $\mathcal{Q}_{g}$ consisting of pairs $(\mathcal{S}, q)$ such that $q$ possesses exactly $n$ singularities on $\mathcal{S}$ with multiplicities $\left(k_{1}, \ldots, k_{n}\right), k_{i} \geq-1$.

Note that the Gauss-Bonnet formula implies that the sum of the multiplicities $\sum k_{i}$ equals $2 g-2$ in the case of $\mathcal{H}\left(k_{1}, \ldots, k_{n}\right)$ and $4 g-4$ in the case of $\mathcal{Q}\left(k_{1}, \ldots, k_{n}\right)$. In Section 2.1.1, we will present Thurston's approach to these surfaces via the theory of measured foliations.

From these definitions, it is a well known part of the Teichmüller theory that these spaces are (Hausdorff) complex analytic, and in fact algebraic, spaces (see [2] for a nice description of the stratum $\mathcal{Q}(1, \ldots, 1)$; see also [5], [9], [23]). Basically, one can see that as follows. We first concentrate on the strata of the moduli spaces $\mathcal{H}_{g}$.

Let $\left(\mathcal{S}, \omega^{2}\right)$ be a representative of an element in $\mathcal{H}\left(k_{1}, \ldots, k_{n}\right), S$ its underlying topological surface, and $P_{1}, \ldots, P_{n}$ its singular points. Let us denote by hol $=\operatorname{hol}_{(\mathcal{S}, \omega)}$ the group morphism $H_{1}\left(S,\left\{P_{1} \ldots, P_{n}\right\}, \mathbb{Z}\right) \rightarrow \mathbb{C}$ defined by $\operatorname{hol}([\gamma])=\int_{\gamma} \omega$ for every 1 -cycle $\gamma$ in $S$ relative to $\left\{P_{1} \ldots, P_{n}\right\}$. Fix a basis $\left(\gamma_{1}, \ldots, \gamma_{2 g+n-1}\right)$ of the free abelian group $H_{1}\left(S,\left\{P_{1} \ldots, P_{n}\right\}, \mathbb{Z}\right)$. Any other element of $\mathcal{H}\left(k_{1}, \ldots, k_{n}\right)$ will be represented by an element having the same underlying surface and the same singular points. With these notations, the map

$$
\Phi=\left(\begin{array}{ccc}
\mathcal{H}\left(k_{1}, k_{2}, \ldots, k_{n}\right) & \longrightarrow & H^{1}\left(S,\left\{P_{1}, \ldots, P_{n}\right\}, \mathbb{C}\right) \\
\mathcal{S}^{\prime} & \longmapsto\left(\gamma_{1}, \ldots, \gamma_{2 g+n-1}\right) \mapsto\left(h_{\mathcal{S}^{\prime}}\left(\gamma_{1}\right), \ldots, \text { hol }_{\mathcal{S}^{\prime}}\left(\gamma_{2 g+n-1}\right)\right)
\end{array}\right)
$$

is named the period map and is a local homeomorphism in a neighbourhood of $\left(\mathcal{S}, \omega^{2}\right)$. Therefore we get a locally one-to-one correspondence between the corresponding stratum of $\mathcal{H}_{g}$ and an open domain in the vector space $H^{1}\left(S,\left\{P_{1}, \ldots, P_{n}\right\} ; \mathbb{C}\right) \simeq \mathbb{C}^{2 g+n-1}$. The changes of coordinates are affine maps outside the singularities of $\mathcal{H}\left(k_{1}, k_{2}, \ldots, k_{n}\right)$ and produce after a study of the singularities a differentiable orbifold structure on the strata of $\mathcal{H}_{g}$.

Let us now consider the case of a stratum of the moduli space $\mathcal{Q}_{g}$. For every $(\mathcal{S}, q) \in \mathcal{Q}_{g}$, consider the canonical double cover $\pi: \hat{\mathcal{S}} \rightarrow \mathcal{S}$ such that $\pi^{*} q=\omega^{2}$ for some holomorphic Abelian differential $\omega$ on $\hat{\mathcal{S}}$ (see for instance [12]). As above, we consider the period map between a neighborhood of the point $(\hat{\mathcal{S}}, \omega)$ and an open subset of $H^{1}\left(\hat{S},\left\{\hat{P}_{1}, \ldots, \hat{P}_{n}\right\} ; \mathbb{C}\right)$. The covering involution $\tau: \hat{\mathcal{S}} \rightarrow \hat{\mathcal{S}}$ induces an involutive linear map on this cohomology vector space. Therefore, this vector space decomposes in two eigenspaces for $\tau^{*}$, say $E_{-1}$ and $E_{+1}$, with eigenvectors -1 and +1 . Abelian differentials in $E_{-1}$ are precisely those which arise from quadratic differentials on $\mathcal{S}$ by pull-back by $\pi$. Hence we obtain a one-to-one correspondence between a neighborhood of any point in the corresponding strata of $\mathcal{Q}_{g}$ and an open domain of $E_{-1} \simeq \mathbb{C}^{2 g+n-2}$.

We next recall the construction of a measure $\mu$ defined on each stratum. For that, the tangent space to $\mathcal{H}_{g}$ (respectively $\mathcal{Q}_{g}$ ) at each point contains a lattice:

$$
H^{1}\left(\mathcal{S},\left\{P_{1}, \ldots, P_{n}\right\} ; \mathbb{Z}\right) \oplus i \cdot H^{1}\left(\mathcal{S},\left\{P_{1}, \ldots, P_{n}\right\} ; \mathbb{Z}\right) \subset H^{1}\left(\mathcal{S},\left\{P_{1}, \ldots, P_{n}\right\} ; \mathbb{C}\right) .
$$

We define a measure $\mu$ on each stratum by pulling back the Lebesgue measure defined on $H^{1}\left(\mathcal{S},\left\{P_{1}, \ldots, P_{n}\right\} ; \mathbb{C}\right)$ normalized so that the volume of the unit cube is 1. 
Let us define the function $A: \mathcal{H}_{g} \rightarrow \mathbb{R}_{+}$by the formula $A(\mathcal{S}, \omega)=\frac{i}{2} \int_{\mathcal{S}} \omega \wedge \bar{\omega}$. This is the area of $\mathcal{S}$ in terms of the flat metric associated to $\omega$.

The group $\mathrm{SL}_{2}(\mathbb{R})$ acts by linear transformations with constant coefficients on the pair of real-valued 1-forms $(\operatorname{Re}(\omega), \operatorname{Im}(\omega))$. In the local affine coordinates, this action is the action of $\mathrm{SL}_{2}(\mathbb{R})$ on the coefficient field of the cohomology vector space $H^{1}\left(S,\left\{P_{1}, \ldots, P_{n}\right\} ; \mathbb{C}\right)$. From this description, it is clear that the subgroup $\mathrm{SL}_{2}(\mathbb{R})$ preserves the measure $\mu$ and the function $A$.

One can define an induced measure $\mu^{(r)}$ on each hypersurface $\mathcal{H}^{(r)}\left(k_{1}, \ldots, k_{n}\right)=A^{-1}(r)$ in the following way. If $E$ is a subset of $\mathcal{H}^{(r)}\left(k_{1}, \ldots, k_{n}\right)$ then we define

$$
\mu^{(r)}(E)=\mu(\{\lambda(\mathcal{S}, \omega) ;(\mathcal{S}, \omega) \in E \text { and } 0<\lambda \leq r\}) .
$$

We also define in an analogous manner a measure $\mu^{(r)}$ on each stratum of $\mathcal{Q}_{g}^{(r)}$.

Recall that each stratum carries a complex algebraic orbifold structure modeled on the first relative cohomology group (see for instance [9]). The dimensions are respectively given by

$$
\begin{array}{lll}
\operatorname{dim}_{\mathbb{C}} \mathcal{H}\left(k_{1}, \ldots, k_{n}\right)=2 g+n-1 & \text { where } & k_{1}+\cdots+k_{n}=2 g-2 \\
\operatorname{dim}_{\mathbb{C}} \mathcal{Q}\left(k_{1}, \ldots, k_{n}\right)=2 g+n-2 & \text { where } & k_{1}+\cdots+k_{n}=4 g-4
\end{array}
$$

The action of the 1-parameter subgroup of diagonal matrices $g_{t}:=\operatorname{diag}\left(e^{t / 2}, e^{-t / 2}\right)$ presents a particular interest for our purpose. It gives a measure-preserving flow for $\mu^{(1)}$ on each stratum. This flow is known as the Teichmüller geodesic flow. Note that orbits under $g_{t}$ project to Teichmüller geodesics on the moduli space of Riemann surfaces $\mathcal{M}_{g}$. The next fundamental result motives our study (see [14], [21, 22, 23]).

Theorem (Masur, Veech). - The Teichmüller geodesic flow acts ergodically on each connected component of each stratum of $\mathcal{Q}_{g}^{(1)}$ with respect to the measure $\mu^{(1)}$, which is finite and in the Lebesgue class.

A direct corollary of the finiteness of the measure $\mu^{(1)}$ on any stratum of $\mathcal{H}_{g}^{(1)}$ is a proof of the Keane's conjecture (see [6]): almost all intervals exchange transformations are uniquely ergodic.

Remark 1.3. - This theorem was proved by Masur [14] for the $\mathcal{Q}(4 g-4)$ case and by Veech [21] for the $\mathcal{H}\left(k_{1}, \ldots, k_{n}\right)$ case.

The ergodicity of the Teichmüller geodesic flow is proved in full generality in [22], Theorem 0.2. The finiteness of the measure $\mu^{(1)}$ appears in two 1984 preprints of Veech: Dynamical systems on analytic manifolds of quadratic differentials I,II (see also [22] p.445). These preprints have been published in 1990 [23]. 
1.1.2. Topology of the Moduli Space. - Following the theorem of Masur and Veech, we are interested in a classification of the connected components of the strata of $\mathcal{H}_{g} \sqcup \mathcal{Q}_{g}$. Veech and Arnoux discovered, by direct calculations in terms of extended Rauzy classes, that some strata are non-connected. They have proved that $\mathcal{H}(2)$ is connected, $\mathcal{H}(4)$ have 2 connected components and $\mathcal{H}(6)$ have 3 connected components.

Recently, in the context of moduli space of Abelian differential $\mathcal{H}_{g}$, Kontsevich and Zorich (see [8]) obtained the following complete description.

THEOREM (Kontsevich, Zorich). - Let $g \geq 4$ be any integer. The topology of any stratum of $\mathcal{H}_{g}$ is given by the following list:

- The strata $\mathcal{H}(2 g-2)$ and $\mathcal{H}(2 k, 2 k)$, for any $k \geq 2$, have three connected components.

- Any other stratum $\mathcal{H}\left(2 k_{1}, \ldots, 2 k_{n}\right)$, for any $k_{i} \geq 1$, has two connected components.

- The stratum $\mathcal{H}(2 k-1,2 k-1)$, for any $k \geq 2$, has two connected components.

Any other stratum of Abelian differentials on a surface of genus $g \geq 4$ is non-empty and connected.

The description of connected components for strata of genera $1 \leq g \leq 3$ is similar to the previous one with some exceptions; we do not present the result here (see [8]). Roughly speaking, Kontsevich and Zorich used two invariants to obtain this classification: the parity of the spin structure and the hyperellipticity.

In [12], we proved that the first invariant extends trivially to the moduli space $\mathcal{Q}_{g}$. However, the second invariant produces non-trivial values. In order to present our statement, we will recall briefly this construction in Section 1.1.3.

In this paper, we will show that this (hyperelliptic) invariant is complete in genera $g \geq 5$ : it classifies precisely the components of the strata of $\mathcal{Q}_{g}$. For small genera, we obtain a similar result with 4 additional mysterious components.

REMARK 1.4. - As a direct corollary of our result and Kontsevich-Zorich's theorem, we conclude that the ergodic components of the Teichmüller geodesic flow are given by an explicit list. In particular, if $n \geq 5$, for any $g \geq 0$, any stratum of $\mathcal{H}_{g} \sqcup \mathcal{Q}_{g}$ with $n$ singularities is connected.

REMARK 1.5. - This paper achieves the classification of connected components of the strata of the moduli spaces $\mathcal{H}_{g} \sqcup \mathcal{Q}_{g}$ announced in the vast program in [9]. Nevertheless a more precise description of the strata remains open. Observe that each hyperelliptic component is isomorphic to a configuration space, and thus we know perfectly well the fundamental group. There is a general conjecture due to Kontsevich for the other connected components.

CONJECTURE (Kontsevich). - Each connected component of the strata of the moduli space $\mathcal{H}_{g} \sqcup \mathcal{Q}_{g}$ is a $K(\pi, 1)$, where $\pi$ is a group commensurable with some mapping class group.

$4^{\mathrm{e}}$ SÉRIE - TOME $41-2008-\mathrm{N}^{\mathrm{o}} 1$ 
1.1.3. Hyperelliptic components. - We will need the following statement. A proof can be found in [8]; see also [11].

Proposition 1.6. - Any stratum of the moduli space $\mathcal{Q}\left(k_{1}, \ldots, k_{n}\right)$ in genus 0 is connected.

Let $\mathcal{S}_{g}$ be a (compact, connected) Riemann surface endowed with a (integrable, meromorphic) quadratic differential $q_{0}$ which is not the global square of any Abelian differential. Let $\left(k_{1}, \ldots, k_{n}\right)$ be its singularity pattern. We do not exclude the case when some of $k_{i}$ are equal to zero: by convention this means that we have some marked points. We shall use the "exponential" notation to denote multiple singularities (simple poles), for example $\mathcal{Q}\left(-1^{5}, 1\right):=\mathcal{Q}(-1,-1,-1,-1,-1,1)$.

Let $\pi: \tilde{\mathcal{S}}_{\tilde{g}} \rightarrow \mathcal{S}_{g}$ be a (ramified) covering such that the image of any ramification point of $\pi$ is a marked point, or a zero, or a pole of the quadratic differential $q_{0}$. Fix the combinatorial type of the covering $\pi$ : the degree of the covering, the number of critical fibers and the ramification index of the points in every critical fiber. Consider the induced quadratic differential $\pi^{*} q_{0}$ on $\tilde{\mathcal{S}}_{\tilde{g}}$; let $\left(\tilde{k}_{1}, \ldots, \tilde{k}_{m}\right)$ be its singularity pattern.

Deforming slightly the initial point $\left(\mathcal{S}_{g}, q_{0}\right) \in \mathcal{Q}\left(k_{1}, \ldots, k_{n}\right)$, we get a ramified covering over the deformed Riemann surface with the same combinatorial type as the covering $\pi$. The induced quadratic differential $\pi^{*} q$ has the same singularity pattern $\left(\tilde{k}_{1}, \ldots, \tilde{k}_{m}\right)$ as $\pi^{*} q_{0}$. Thus we obtain a map from the stratum $\mathcal{Q}\left(k_{1}, \ldots, k_{n}\right)$ to the stratum $\mathcal{Q}\left(\tilde{k}_{1}, \ldots, \tilde{k}_{m}\right)$. We will denote this map by $\Pi$. In [11], we proved that $\Pi$ is an immersion.

Now we recall an example of this construction for strata having four singularities. Consider a meromorphic quadratic differential $q$ on $\mathbb{C} P(1)$ having the singularity pattern $(2(g-$ $k)-3,2 k+1,-1^{2 g+2}$ ), where $k \geq-1, g \geq 1$ and $g-k \geq 2$. Consider a ramified double covering $\pi$ over $\mathbb{C} P(1)$ having ramification points over the $2 g+2$ poles of $q$, and no other ramification points. We obtain a hyperelliptic Riemann surface $\tilde{\mathcal{S}}$ of genus $g$ with a quadratic differential $\pi^{*} q$ on it. A straightforward computation shows that the induced quadratic differential $\pi^{*} q$ has the singularity pattern $(2(g-k)-3,2(g-k)-3,2 k+1,2 k+1)$. Thus we get a map

$$
\mathcal{Q}\left(2(g-k)-3,2 k+1,-1^{2 g+2}\right) \rightarrow \mathcal{Q}(2(g-k)-3,2(g-k)-3,2 k+1,2 k+1),
$$

where $k \geq-1, g \geq 1$ and $g-k \geq 2$. Computing the dimensions of the strata, we get

$$
\begin{aligned}
& \operatorname{dim}_{\mathbb{C}} \mathcal{Q}\left(2(g-k)-3,2 k+1,-1^{2 g+2}\right)=2 \cdot 0+(2 g+4)-2=2 g+2 \\
& \operatorname{dim}_{\mathbb{C}} \mathcal{Q}(2(g-k)-3,2(g-k)-3,2 k+1,2 k+1)=2 g+4-2=2 g+2
\end{aligned}
$$

Since the dimension of the strata coincide, and the mapping is an immersion, we obtain an open set inside the stratum $\mathcal{Q}(2(g-k)-3,2(g-k)-3,2 k+1,2 k+1)$. Note that the action of the geodesic flow is equivariant, thus by ergodicity the image of this map has full measure and contains only hyperelliptic curves. Therefore it is onto. This remark, together with Proposition 1.6, motivates the following definition. We call $\mathcal{Q}^{\text {hyp }}(2(g-k)-3,2(g-k)-$ $3,2 k+1,2 k+1)$ the hyperelliptic connected component of the stratum $\mathcal{Q}(2(g-k)-3,2(g-$ $k)-3,2 k+1,2 k+1)$ consisting of the quadratic differentials on genus $g$ hyperelliptic curves that are images by the above map of quadratic differentials on the sphere with singularities data $\left(2(g-k)-3,2 k+1,-1^{2 g+2}\right)$. 
In [11], we classify all components of this type. This motivates the following definition.

Definition. - By hyperelliptic components, we mean the following connected components of the following strata of quadratic differentials.

1. The connected component $\mathcal{Q}^{\text {hyp }}(2(g-k)-3,2(g-k)-3,2 k+1,2 k+1)$ of the stratum $\mathcal{Q}(2(g-k)-3,2(g-k)-3,2 k+1,2 k+1)$ consisting of the images of the pull-back of the quadratic differentials of the stratum $\mathcal{Q}\left(2(g-k)-3,2 k+1,-1^{2 g+2}\right)$ where $k \geq-1, g \geq 1, g-k \geq 2$.

The corresponding ramified double covering has ramification points over the $2 g+2$ poles.

2. The connected component $\mathcal{Q}^{\text {hyp }}(2(g-k)-3,2(g-k)-3,4 k+2)$ of the stratum $\mathcal{Q}(2(g-k)-3,2(g-k)-3,4 k+2)$ consisting of the images of the pull-back of the quadratic differentials of the stratum $\mathcal{Q}\left(2(g-k)-3,2 k,-1^{2 g+1}\right)$ where $k \geq 0, g \geq 1$ and $g-k \geq 1$.

The corresponding ramified double covering has ramification points over the $2 g+1$ poles and over the zero of degree $2 k$.

3. The connected component $\mathcal{Q}^{\text {hyp }}(4(g-k)-6,4 k+2)$ of the stratum $\mathcal{Q}(4(g-k)-$ $6,4 k+2)$ consisting of the images of the pull-back of the quadratic differentials of the stratum $\mathcal{Q}\left(2(g-k)-4,2 k,-1^{2 g}\right)$ where $k \geq 0, g \geq 2$ and $g-k \geq 2$.

The corresponding double ramified covering has ramification points over all the singularities.

REMARK 1.7. - Hyperelliptic connected components of type (1) were first discovered by Kontsevich.

1.1.4. Main result. - We are finally in a position to give a precise statement of our result.

Theorem 1.1. - Let $g \geq 5$ be any integer. Let us consider the following families $\mathcal{F}_{i}, i=$ $2,3,4$, of strata inside the moduli space of integrable meromorphic quadratic differentials $\mathcal{Q}_{g}$.

$$
\begin{array}{ll}
\mathcal{F}_{2}=\{\mathcal{Q}(4(g-k)-6,4 k+2) & 0 \leq k \leq g-2\} \\
\mathcal{F}_{3}=\{\mathcal{Q}(2(g-k)-3,2(g-k)-3,4 k+2) & 0 \leq k \leq g-1\} \\
\mathcal{F}_{4}=\{\mathcal{Q}(2(g-k)-3,2(g-k)-3,2 k+1,2 k+1) & \mid-1 \leq k \leq g-2\}
\end{array}
$$

Then any stratum listed above has exactly two connected components: one is hyperelliptic the other not.

Any other stratum of the moduli space $\mathcal{Q}_{g}$ is non-empty and connected.

In small genera, some components are missing compared to the general case. The complete description is given by the following theorem.

THEOREM 1.2. - Let $g \leq 4$ be any non-negative integer. The components of the strata of the moduli space $\mathcal{Q}_{g}$ can be described as follows.

- In genera 0 and 1 , any stratum is non-empty and connected, except $\mathcal{Q}(0)$ and $\mathcal{Q}(-1,1)$ that are empty. 
- In genus 2 , there are two non-connected strata: $\mathcal{Q}(-1,-1,6)$ and $\mathcal{Q}(-1,-1,3,3)$; they have two connected components, one component is hyperelliptic the other not. There are two empty strata: $\mathcal{Q}(4)$ and $\mathcal{Q}(1,3)$. Any other stratum of $\mathcal{Q}_{2}$ is non-empty and connected.

- In genera 3 and 4, each stratum possessing a hyperelliptic connected component has exactly two connected components: one is hyperelliptic, the other not.

- There are 4 sporadic strata:

$$
\mathcal{Q}_{3}(-1,9), \mathcal{Q}_{3}(-1,3,6), \mathcal{Q}_{3}(-1,3,3,3), \mathcal{Q}_{4}(12)
$$

that have exactly two connected components.

- Any other stratum of $\mathcal{Q}_{3}$ and $\mathcal{Q}_{4}$ is non-empty and connected.

REMARK 1.8. - The fact that the four sporadic strata are not connected has been proved by Zorich (see [25]). Zorich proved this result by a direct calculation in terms of extended Rauzy classes on generalized permutations (see also [1]). In this paper we only prove that theses strata have at most two connected components.

It would be interesting to have an algebro-geometric proof of the non-connectedness of these strata.

REMARK 1.9. - Using our approach of quadratic differentials, we get a new proof of a result of Masur and Smillie [15] concerning the fact that the strata $\mathcal{Q}(0), \mathcal{Q}(-1,1), \mathcal{Q}(1,3)$ and $\mathcal{Q}(4)$ are empty.

\subsection{Outline of the proof}

The proof involves the dynamics and topology of measured foliations. We will use the well known relation between quadratic differentials $q$ on $\mathcal{S}$ and pair of transverse measured foliations on $\mathcal{S}$ (see e.g. [5]). We will say that a component $\mathcal{C}_{1}$ of a strata of $\mathcal{Q}_{g}$ is adjacent to a component $\mathcal{C}_{2}$ if $\mathcal{C}_{2} \subset \overline{\mathcal{C}_{1}}$ where the closure is taken inside the whole space $\mathcal{Q}_{g}$.

1 (Claim A). - Let $\mathcal{C}$ be any component of any stratum $\mathcal{Q}_{g}\left(k_{1}, \ldots, k_{n}\right)$ with $n \geq 2$ and $g \geq 3$. We assume that $\mathcal{C}$ is neither a hyperelliptic component nor a sporadic component $\mathcal{Q}^{\text {irr }}(-1,9), \mathcal{Q}^{\text {irr }}(-1,3,6)$ or $\mathcal{Q}^{\text {irr }}(-1,3,3,3)$ (to be defined later on). Then $\mathcal{C}$ is adjacent to a connected component of the stratum $\mathcal{Q}(4 g-4)$. We postpone the idea of a proof in $\mathbf{6}$.

2. - We will use the following corollary of a theorem of Konsevich (see also [8]): The number of connected components of a stratum of $\mathcal{Q}_{g}$, which are adjacent to a component of the stratum $\mathcal{Q}(4 g-4)$, is bounded from above by the number of components of $\mathcal{Q}(4 g-4)$. In addition, for a given stratum, there is at most one hyperelliptic or one sporadic component. These two remarks, together with Claim A, provide us a bound on the number of components of any stratum in terms of the number $r_{g}$ of components of $\mathcal{Q}(4 g-4)$. More precisely, the number of components of a stratum containing a hyperelliptic component (or a sporadic component) is bounded from above by $r_{g}+1$. The number of components of a stratum containing nor a hyperelliptic component neither a sporadic component is bounded from above by $r_{g}$. 
3. - The list of strata which possess a hyperelliptic component has been given in [11]. In particular, these strata are non connected for $g \geq 3$. Hence the proof of Theorem 1.1 is reduced to the proof of the equality $r_{g}=1$ for any $g \geq 5$.

Theorem 1.2 follows from the equality $r_{3}=1$ and the inequality $r_{4} \leq 2$ that we prove in Section 6. The small genera cases $g=1,2$ are considered separately; this is done in Section 8 .

Therefore the proof of our result is reduced to the one of Claim A and to the computation of $r_{g}$.

We will say that a saddle connection $\gamma$ on $(\mathcal{S}, q)$ has multiplicity one (see [17]) if one can collapse $\gamma$ to a point to get a new non degenerated flat surface $\mathcal{S}^{\prime}$. In particular, this condition is satisfied if $\gamma$ is "small" with respect to the other saddle connections in the direction of $\gamma$. More precisely it follows from results in [17]

$$
|\gamma|<\frac{1}{2}|\eta| \text {, for any saddle connection } \eta \text {, if any, on } \mathcal{S} \backslash \gamma \text { in the direction } \vec{\gamma}
$$

then $\gamma$ has multiplicity one. We will give a useful criterion on flat surfaces to have such an inequality.

4. - Let $(\mathcal{S}, q) \in \mathcal{C}$ and $\gamma$ be a multiplicity one saddle connection of $(\mathcal{S}, q)$. Then $\gamma$ can be collapsed to a point to get a new flat surface $\left(\mathcal{S}^{\prime}, q^{\prime}\right)$. The component $\mathcal{C}^{\prime}$ containing $\left(\mathcal{S}^{\prime}, q^{\prime}\right)$ is contained in $\overline{\mathcal{C}}$.

5. - Let us assume that $\mathcal{S}$ is decomposed in a single cylinder for the horizontal direction. It means that any two regular horizontal geodesics are closed and homologous: they form a family which fill the surface in a metric cylinder. The boundaries of this cylinder consist of a set of saddle connections and separatrix loops. The arrangement of these separatrices is described by a "generalized permutation" $\pi$ (see Section 3). We will note $(\mathcal{S}, q)=\mathcal{S}(\pi)$ for a one cylinder surface. In Section 5, we give a combinatorial criterion (namely irreducibility) on the combinatorics of $\pi$ so that there exists a saddle connection satisfying Equation (1.1). In particular this produces a multiplicity one saddle connection.

6. - The proof of Claim $A$ is the following. Let $\mathcal{S}(\pi) \in \mathcal{C}$ be a point in an arbitrary component (such points are dense in each stratum of the moduli space $\mathcal{Q}_{g}$ ). We consider the set of surfaces $\mathcal{S}\left(\pi_{s}\right):=h_{s} \cdot \mathcal{S}(\pi)$ where $h_{s}=\left(\begin{array}{ll}1 & s \\ 0 & 1\end{array}\right)$.

If $\mathcal{S}\left(\pi_{s}\right)$ has a multiplicity one saddle connection for some $s$, we are done thanks to 4 . Otherwise the generalized permutation $\pi_{s}$ must obey to some combinatorial conditions. Repeating that for any $s$ we get that either $\mathcal{S}(\pi)$ has a multiplicity one saddle connection or $\pi$ is an exceptional (or hyperelliptic) permutation. The last case implies that $\mathcal{C}$ is an exceptional (or hyperelliptic) component contradicting the assumptions of Claim A.

7. - The proof of the connectedness of $\mathcal{Q}(4 g-4)$ (i.e. of the equality $r_{g}=1$ ) for $g \geq 5$ is done by induction on the genus $g$ of the surfaces. We first show by a direct computation that the minimal stratum in genus 5 is connected. We then use the surgery "Bubbling a handle" for the step of the induction (see [8]). Precisely, we find in each connected component a surface with a cylinder filled by closed geodesic such that the boundary is formed by two single multiplicity one saddle connections. Therefore one can "erase" this cylinder to obtain a closed $(g-1)$ flat surface and get the induction process. 
The paper is organized in the following manner. In Section 2 we remind key results concerning the geometry of quadratic differentials. Then Sections 3-5 are devoted to the notion of generalized permutations. We develop this useful notion and give some relations between the combinatorics of $\pi$ and the dynamic of the measured foliation on $\mathcal{S}(\pi)$. Finally in Section 6 and Section 7 we prove respectively the two points 7 and 6 .

\subsection{Remarks and Acknowledgments}

This paper is the last part of my $\mathrm{PhD}$ thesis, the first two parts being the papers [11] and [12]. I would like to thank Pascal Hubert and Anton Zorich for encouraging me to write this paper.

I also thank Pascal Hubert, Maxim Kontsevich, Howard Masur, Anton Zorich and the anonymous referee for helpful comments on preliminary versions of this manuscript.

I thank the Institut de Mathématiques in Luminy, the Max-Planck-Institute for Mathematics in Bonn and the Centre de Physique Théorique in Marseille for excellent welcome and working conditions.

\section{Preliminaries and preparatory material}

In order to establish notations and preparatory material, we review basic notions concerning quadratic differentials versus flat surfaces. These surfaces have been considered and studied by numerous authors in various guises, see say [5], [7], [19] for more details; See also [3] and [17] for recent related developments on surgeries about flat surfaces.

\subsection{Flat metrics}

2.1.1. Flat surfaces and geodesics. - A flat surface is a (compact, connected, real) genus $g$ surface equipped with a flat metric (with isolated conical singularities) such that the holonomy group belongs to $\{ \pm \mathrm{Id}\}$. Here holonomy means that the parallel transport of a vector along a small loop going around a conical point brings the vector back to itself or to its negative. This implies that all cone angles are integer multiples of $\pi$. An equivalent definition is the following. A flat surface is a triple $(\mathcal{S}, \mathcal{U}, \Sigma)$ such that $\mathcal{S}$ is a topological compact connected surface, $\Sigma$ is a finite subset of $\mathcal{S}$ (whose elements are called singularities) and $\mathcal{U}=$ $\left(U_{i}, z_{i}\right)$ is an atlas of $\mathcal{S} \backslash \Sigma$ such that the transition maps $z_{j} \circ z_{i}^{-1}: z_{i}\left(U_{i} \cap U_{j}\right) \rightarrow z_{j}\left(U_{i} \cap U_{j}\right)$ are translations or symmetries: $z_{i}= \pm z_{j}+$ const. This implies that the holonomy belongs to $\{ \pm \operatorname{Id}\}$.

Therefore, we get on $\mathcal{S}$ a flat metric with conical singularities located in $\Sigma$ (possibly not all $\Sigma$ ). We also get a quadratic differential defined locally in the coordinates $z_{i}$ by the formula $q=d z_{i}^{2}$. This form extends to the points of $\Sigma$ to zeroes, poles or marked points (see [16]). We will sometimes use the notation $(\mathcal{S}, q)$ or simply $\mathcal{S}$.

REMARK 2.1. - The holonomy is trivial if and only if all transition functions are translations or equivalently the quadratic differentials $q$ is the square of an Abelian differential. We will then say that $\mathcal{S}$ is a translation surface. 
Hence the flat structure defines on $\mathcal{S} \backslash \Sigma$ a Riemannian structure; we therefore have notions of directional foliation, geodesic, length, angle, measure, etc.

\section{Convention}

- The singularities are the zeroes and poles of $q$.

- Leaves of the directional foliation are called geodesics.

- Geodesics meeting singularities are called separatrices. A geodesic emanating from a singularity and going back to the same is called a separatrix loop. A geodesic connecting two different singularities is called a saddle connection.

- A geodesic not passing through a singularity is called regular.

One can see the equivalence between flat surface and quadratic differential as follows. We start with the first definition. If we cut a flat surface $\mathcal{S}$ successively along an appropriate collection of saddle connections, we can decompose it in polygons contained in $\mathbb{C}$. We may then view $\mathcal{S}$ as a union of polygons with sides ordered by pairs consisting of parallels sides of the same length. The surface $\mathcal{S}$ is then isometric to the polygons where we identify these pair by translations or symmetries. Note that we have endowed each polygon with a complex coordinate. By construction, the transition functions in these complex coordinates $z$ have the form

$$
z \mapsto \pm z+\text { const. }
$$

Thus any flat surface with the conical singularities removed is endowed with a natural complex structure. Moreover, consider a holomorphic quadratic differential $q=d z^{2}$ on every polygon. Since $d z^{2}=d( \pm z+\text { const })^{2}$ we obtain a globally well defined holomorphic quadratic differential on $\mathcal{S}^{\prime}$. It is a direct calculation to check that the complex structure and the quadratic differential can be extended to the singularities; the quadratic differential $q$ extends to a (possibly meromorphic) form on $\mathcal{S}$ with zeroes or simple poles at every conical point. Note that when all transitions function are only translations, the quadratic differential $q$ can be globally written as $q=\omega^{2}$, where $\omega$ is an Abelian differential. In this case the corresponding foliation is oriented.

Conversely, given a pair $(\mathcal{S}, q)$, and a point $P \in \mathcal{S}$ such that $q(P) \neq 0$, the integral $\int_{z_{0}}^{z} \sqrt{q}$ produces a local coordinate $z$ near $P$ such that $q=d z^{2}$. Thus $|d z|^{2}$ defines a flat metric on $\mathcal{S}$. At a singularity of multiplicity $k \geq-1$ the total angle we get is $(k+2) \pi$. Remark that for regular point of $q(k=0)$, one get regular point of the metric.

2.1.2. $\mathrm{SL}_{2}(\mathbb{R})$-action. - Given any matrix $A \in \mathrm{SL}_{2}(\mathbb{R})$, we can post-compose the local coordinate of the charts of our translation atlas on $(\mathcal{S}, q)$ by $A$. One easily checks that this gives a new flat surface, denoted by $A \cdot(\mathcal{S}, q)$. In local coordinates, this gives

$$
A \cdot\left(\mathcal{S}, d z^{2}\right)=\left(\mathcal{S},(d A z)^{2}\right) .
$$

Hence this produces an $\mathrm{SL}_{2}(\mathbb{R})$-action on these flat surfaces.

$4^{\mathrm{e}}$ SÉRIE - TOME $41-2008-\mathrm{N}^{\mathrm{o}} 1$ 
2.1.3. Cylinders. - Closed regular geodesics appear in families of parallel geodesics of the same length. Such parallel closed geodesic, typically, do not filled the surface, but only a cylindrical subset. Each boundary component of such a cylinder is comprised by saddle connections.

Generically, each boundary component of a cylinder filled with closed regular geodesics is a single closed saddle connection. The converse, however, is false. A closed saddle connection does not necessarily bound a cylinder of regular closed geodesics. In fact, it bounds such a cylinder if and only if the angle at the singularities between the outgoing and incoming segments is exactly $\pi$. One calls such a cylinder a simple cylinder (see Section 6). See also Figure 7.

2.1.4. Adjacency. - Let $\mathcal{C}, \mathcal{C}^{\prime}$ be two connected components of the moduli space $\mathcal{Q}_{g}$. We will say that $\mathcal{C}^{\prime}$ is adjacent to $\mathcal{C}$ if $\mathcal{C} \subset \overline{\mathcal{C}^{\prime}}$ (the closure being taken inside the whole space $\mathcal{Q}_{g}$ ).

We end this section with an example of flat surface with Figure 3. Identifying pairs of sides of the polygon by isometries, we get a flat surface of genus $g=1$. Note the form $d z$ is not globally defined but $d z^{2}$ is; therefore the holonomy is exactly $\{ \pm \mathrm{Id}\}$.

\subsection{Homologous saddle connections}

Let $\mathcal{S}$ be a flat surface. We denote by $\pi: \hat{\mathcal{S}} \rightarrow \mathcal{S}$ the standard orientating double covering so that $\pi^{*} q=\hat{\omega}^{2}$ (see [12]). Let $\tau$ be the induced involution of the covering. Given an oriented saddle connection $\gamma$ on $\mathcal{S}$ let $\gamma^{+}, \gamma^{-}$be its lifts to the double cover. We denote by $P$ the set of singularties of $q$ and $\hat{P}=\pi^{-1}(P)$. If $\left[\gamma^{+}\right]=-\left[\gamma^{-}\right]$as cycles in $H_{1}(\hat{\mathcal{S}}, \hat{P} ; \mathbb{Z})$ we let $[\hat{\gamma}]:=\left[\gamma^{+}\right]$, otherwise we define $[\hat{\gamma}]$ as $[\hat{\gamma}]:=\left[\gamma^{+}\right]-\left[\gamma^{-}\right]$.

2.2.1. Homologous saddle connections. - Following Masur and Zorich [17], we say that the saddle connections $\gamma, \eta$ on a flat surface $\mathcal{S}$ defined by a quadratic differential $q$ are homologous if $[\hat{\gamma}]=[\hat{\eta}]$ in $H_{1}(\hat{\mathcal{S}}, \hat{P} ; \mathbb{Z})$ under an appropriate choice of orientations of $\gamma, \eta$.

Note that this definition does not depend of the choice of the orientation of the geodesics neither the choice of a direction on $\mathcal{S}$. Moreover, $\gamma$ and $\eta$ are not supposed to be homeomorphic to a circle. For instance, one can have a saddle connection (homeomorphic to a segment) homologous to a separatrix loop (homeomorphic to a circle); see Figure 3. The following proposition gives a necessary condition for two compact separatrices to be homologous.

Proposition 2.2 (Masur, Zorich). - Let us assume that $\gamma$ and $\eta$ are two saddle connections. If $\gamma$ and $\eta$ are homologous then they are parallel and their lengths are equal or differ by a factor two:

$$
\frac{|\gamma|}{|\eta|} \in\left\{1,2, \frac{1}{2}\right\}
$$

ExAmple 2.3. - In Figure 3, one can check that the vertical saddle connection $\gamma(\pi)$ and the vertical separatrix loop $\eta$ are homologous. 
2.2.2. Multiplicity. - Let $\gamma$ be a saddle connections on $\mathcal{S}$. We will say that $\gamma$ has multiplicity $n$, and we will denote $\operatorname{mult}(\gamma)=n$, if there exist exactly $n$ different classes $[\eta] \in H_{1}(\hat{\mathcal{S}}, \hat{P} ; \mathbb{Z})$ where $\eta$ is a saddle connections homologous to $\gamma$.

We will say that a simple cylinder has multiplicity $n$ if the multiplicity of its boundary (which can be represented by a saddle connections) is $n$.

Lemma 2.4. - Let $(\mathcal{S}, q)$ be any flat surface in any hyperelliptic connected component of the following type:

$$
\begin{aligned}
& \mathcal{Q}^{\text {hyp }}(4(g-k)-6,4 k+2) \\
& \mathcal{Q}^{\text {hyp }}(2(g-k)-3,2(g-k)-3,4 k+2) \\
& \mathcal{Q}^{\text {hyp }}(2(g-k)-3,2(g-k)-3,2 k+1,2 k+1)
\end{aligned}
$$

For each of these strata, let $\gamma$ be a saddle connection on $(\mathcal{S}, q)$ between (respectively):

- the zero of degree $4(g-k)-6$ and the one of degree $4 k+2$,

- one of the two zeroes of degree $2(g-k)-3$ and the other zero of degree $4 k+2$,

- one of the two zeroes of degree $2(g-k)-3$ and one of the two zeroes of degree $2 k+1$, $2 k+1$.

Then $\gamma$ has multiplicity at least two.

Proof of the lemma. - The surface $\mathcal{S}$ is hyperelliptic so it is equipped with an hyperelliptic involution, say $\tau$. Take a saddle connection $\gamma$ as indicated in the assumptions. By construction [11], $\gamma_{2}:=\tau(\gamma) \neq \gamma$ thus we obtain an other saddle connection in the same direction of $\gamma$ (and of the same length). Always by construction we get that $\hat{\gamma}=\hat{\gamma}_{2}$. Therefore $\gamma_{2}$ is homologous to $\gamma$ and $[\gamma] \neq \gamma_{2}$ hence $\operatorname{mult}(\gamma) \geq 2$. The lemma is proven.

\subsection{Surgeries}

The principal ingredient of our proof is to decrease the complexity of a stratum. By complexity, we mean the genus $g$ of the surfaces and the number $n$ of the singularities. The next two subsections describe how one can increase the complexity. For details and proofs of the two next sections see [3], [8] and [17]). In Section 5 we will present some results to get the converse.

2.3.1. Breaking up a singularity. - Let $(\mathcal{S}, q)$ be a flat surface and let $P$ be a singularity of $q$. Let $(k+2) \pi$ be the conical angle around $P$ with $k \geq-1$. Choose any partition of $k$ in two non-zero integers $k_{1}, k_{2}$ with $k_{i} \geq-1$. We recall the well known construction to obtain a new flat structure $q^{\prime}$ on $\mathcal{S}$ with the same singularities pattern as $q$ except at the point $P$; The new flat surface will possess two singularities $P_{k_{1}}$ and $P_{k_{2}}$ of multiplicities $k_{1}$ and $k_{2}$. Moreover $\mathcal{S}^{\prime}$ will possess a multiplicity one saddle connection between $P_{k_{1}}$ et $P_{k_{2}}$. Here we detail the case $k$ odd and the case $k_{1}, k_{2}$ even.

Consider a small geodesic neighborhood of $P$, that is an $\varepsilon-$ "polydisc" constructed from $k+2$ half Euclidean discs of radii $\varepsilon$ glued in their centers (see Figure 1, see also also Figure 4 and Figure 5 in [11]).

Now, for $\varepsilon$ small enough, there is no critical geodesic passing through this polydisc, other than the $k+2$ emanating rays from $P$. Let us remove this polydisc and change continuously 

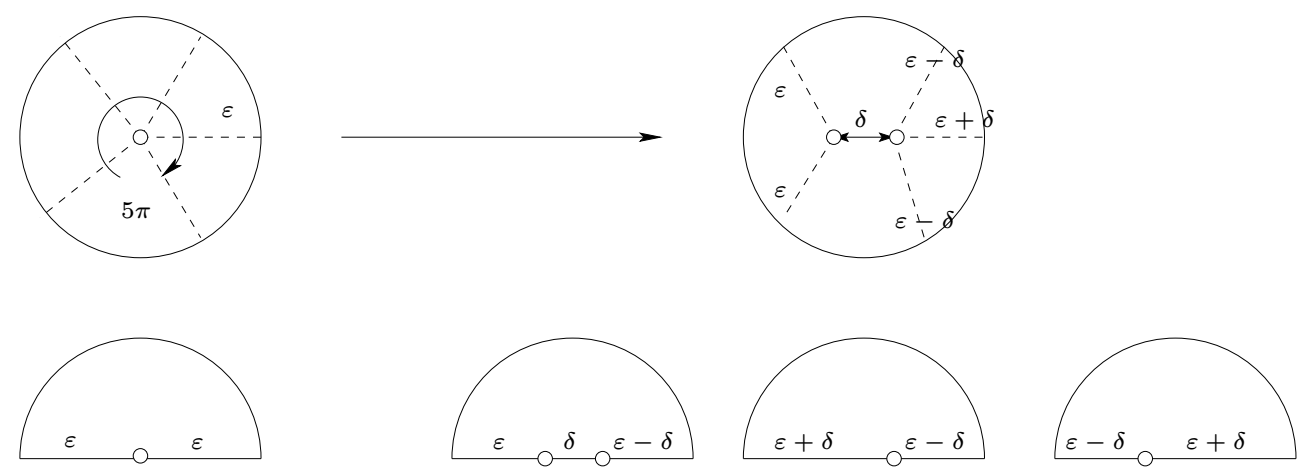

FiguRE 1. Breaking up a zero of order 3 in two zeroes of orders 1 and 2 correspondingly. Note that the surgery is local: we do not change the flat metric outside of the neighborhood of the zero.

the parameters in the following manner. We break up the singularity $P$ in two singularities of conical angle $\left(k_{1}+2\right) \pi$ and $\left(k_{2}+2\right) \pi$. This is possible by the choice of $k_{i}$ (see Figure 1 for details). Now one can re-glue this polydisc on the surface $\mathcal{S}$ to obtain the desired new flat structure on $\mathcal{S}$.

ReMARK 2.5. - The geodesic (for $q^{\prime}$ ) on $\mathcal{S}$ connecting $P_{k_{1}}$ and $P_{k_{2}}$ has multiplicity one. Indeed one can choose its length arbitrary small without changing the others lengths thus Proposition 2.2 applies.

If $\mathcal{C}$ denotes the component which contains $(\mathcal{S}, q)$ and $\mathcal{C}^{\prime}$ denotes the component which contains $\left(\mathcal{S}, q^{\prime}\right)$ then $\mathcal{C}^{\prime}$ is adjacent to $\mathcal{C}$ i.e. $\mathcal{C} \subset \overline{\mathcal{C}^{\prime}}$.

The construction we have presented is local: we do not change the metric outside the $\varepsilon$-polydisc. In the case where the parameters $k_{1}$ and $k_{2}$ are odd, the construction is global. This is the parallelogram construction (see [17]).

Theorem 2.6 (Masur, Zorich). - Let $(\mathcal{S}, q) \in \mathcal{Q}\left(k_{1}, \ldots, k_{n}\right)$ be a point. Let us also assume that there exists a multiplicity one saddle connection on $\mathcal{S}$ between the singularities $P_{1} \in$ $\mathcal{S}$ of order $k_{1}$ and $P_{2} \in \mathcal{S}$ of order $k_{2}$. We will make the additional assumption $\left\{k_{1}, k_{2}\right\} \neq$ $\{-1,-1\}$.

Then there exists a point $\left(\mathcal{S}^{\prime}, q^{\prime}\right) \in Q\left(k_{1}+k_{2}, k_{3}, \ldots, k_{n}\right)$ such that one can break up the singularity $P_{k_{1}+k_{2}}^{\prime} \in \mathcal{S}^{\prime}\left(\right.$ for $\left.q^{\prime}\right)$ in two singularities to obtain the initial flat surface $(\mathcal{S}, q)$.

Proof of Theorem 2.6. - Here we address the proof in cases of $k_{1}, k_{2}$ even or $k_{1}+k_{2}$ odd. Assume that $\gamma$ is a multiplicity one saddle connection between $P_{1}$ and $P_{2}$. As usual we will assume that $\gamma$ is vertical. Using the geodesic flow, we contract it to a short segment of length $\delta$. Choose any $\varepsilon$ with $\frac{\delta}{2}<\varepsilon$. Now consider an $\varepsilon$-polydisc $D(\varepsilon)$ of these two points as indicated in Figure 1. The assumption on the multiplicity of $\gamma$ implies that one can and do choose $\varepsilon$ and $\delta$ small enough so that there is no (vertical) critical geodesics inside $D(\varepsilon)$ other than $\gamma$ and the $k_{1}+2$ and $k_{1}+2$ verticals emanating from $P_{1}$ and $P_{2}$. We can apply above construction to replace this polydisc by a new one, where one has glued the two singularities 
together. The new surface $\left(\mathcal{S}, q^{\prime}\right)$ we have constructed satisfies the conclusions of the theorem: on can break up the singularity $P=P_{1}=P_{2}$ in two to obtain our initial surface $(\mathcal{S}, q)$. The theorem is proven.

From the previous construction one easily gets the following useful result for the next.

COROLlaRY 2.7. - Let $\mathcal{S}$ be a flat surface in the stratum $Q\left(k_{1}+k_{2}, k_{3}, \ldots, k_{n}\right)$. Let us assume that $k_{1}+k_{2} \neq k_{i}$ for all $i=3, \ldots, n$. Then all surfaces obtained from $\mathcal{S}$ by breaking up the singularity of multiplicity $k_{1}+k_{2}$ with discrete parameters $k_{1}, k_{2}$ (and arbitrary continuous parameters) belong to the same connected component of the stratum $Q\left(k_{1}, k_{2}, k_{3}, \ldots, k_{n}\right)$.

In terms of adjacency. Let us assume $\mathcal{C}_{0} \subseteq \mathcal{Q}\left(k_{1}+k_{2}, \ldots, k_{n}\right)$ is a connected component and $\mathcal{C}_{1}, \mathcal{C}_{2} \subset \mathcal{Q}\left(k_{1}, k_{2}, \ldots, k_{n}\right)$ are two components such that $\mathcal{C}_{0} \subset \overline{\mathcal{C}_{1}} \cap \overline{\mathcal{C}_{2}}$ and $k_{1}+k_{2} \neq k_{i}$ for all $i=3, \ldots, n$. Then $\mathcal{C}_{1}=\mathcal{C}_{2}$.

2.3.2. "Bubbling a handle". - Let $(\mathcal{S}, q)$ be a flat surface and $P \in \mathcal{S}$ a singularity of $q$. Let us break up the singularity $P$ in two singularities $P_{1}, P_{2} \in \mathcal{S}$ (see previous section). One gets a new flat structure, say $q_{1}$ on $\mathcal{S}$, and a closed saddle connection $\gamma$ (of length $\delta$ ) between $P_{1}$ and $P_{2}$. Let us cut this surface along $\gamma$. We obtain a surface with some boundaries components. We identify the two points $P_{1}, P_{2}$ on this surface to obtain a surface $\mathcal{S}_{1}$ with a boundary component isometric to the union of two circles, each of length $\delta$. Then let us glue a straight metric cylinder with the following parameters. The height and the twist are chosen arbitrarily, and the weight (circumference) is $\delta$. The new surface $\left(\mathcal{S}^{\prime}, q^{\prime}\right)$ we get is a (genus $(\mathcal{S})+1$ ) flat surface. The angle between the new handle is $k_{1}+2$ (or $k_{2}+2$ if we consider the complementary angle). See Figure 7 for an example.

Let $(\mathcal{S}, q)$ be any flat surface. Assume that the singularities data of $\mathcal{S}$ are either $(4 g-3,-1)$ or $(4 g-4)$. Let $s$ be any non-negative integer. Thanks to Corollary 2.7, the surfaces $\left(\mathcal{S}^{\prime}, q^{\prime}\right)$ constructed by the surgery "bubbling a handle" at the unique zero of $q$ in $\mathcal{S}$ with arbitrary continuous parameters (height, twist and width of the cylinder) and let us fix discrete parameter $s$ represent quadratic differentials belonging in the same connected component of the stratum. This motivates the following definition.

Definition 2.8. - Let $(\mathcal{S}, q)$ be any flat surface, with singularities pattern $(4 g-3,-1)$ or $(4 g-4)$ and let $s$ be any non-negative integer. We will denote by $\mathcal{S} \oplus s$, a surface constructed by the surgery "bubbling a handle" at the unique zero of $q$ in $\mathcal{S}$ with arbitrary continuous parameters (height, twist and width of the cylinder) and discrete parameter $s$. If $\mathcal{C}$ denote the component containing $(\mathcal{S}, q)$ then we denote the component containing $\mathcal{S} \oplus s$ by $\mathcal{C} \oplus s$. In other terms we obtain the two well defined mappings.

$$
\begin{aligned}
& \text { For any } g \geq 2, \quad \oplus=\left(\begin{array}{rl}
\pi_{0}(\mathcal{Q}(4 g-3,-1)) \times & \mathbb{N} \longrightarrow \pi_{0}(\mathcal{Q}(4 g+1,-1)) \\
(\mathcal{C}, s) & \longmapsto \mathcal{C} \oplus s
\end{array}\right) . \\
& \text { For any } g \geq 3, \quad \oplus=\left(\begin{array}{rl}
\pi_{0}(\mathcal{Q}(4 g-4)) \times & \mathbb{N} \longrightarrow \pi_{0}(\mathcal{Q}(4 g)) \\
(\mathcal{C}, s) & \longmapsto \mathcal{C} \oplus s
\end{array}\right) .
\end{aligned}
$$


Here $\pi_{0}(E)$ denotes the set of connected components of the topological space $E$. Note also that the discrete parameter $s$ corresponding to the angle between the two new sectors can be chosen modulo $2 g(s \in\{1, \ldots, 2 g\})$.

Proposition 2.9. - Let $\mathcal{C}$ be any connected component of any above stratum, namely $\mathcal{Q}(-1,4 g-3)$ or $\mathcal{Q}(4 g-4)$. Then the following statements hold.

(1) $\mathcal{C} \oplus s_{1} \oplus s_{2}=\mathcal{C} \oplus s_{2} \oplus s_{1} \quad$ for any $s_{1}, s_{2}$.

(2) $\mathcal{C} \oplus s_{1} \oplus s_{2}=\mathcal{C} \oplus\left(s_{2}-2\right) \oplus\left(s_{1}+2\right)$ for any $s_{2} \geq 3$.

(3) $\mathcal{C} \oplus s_{1} \oplus s_{2}=\mathcal{C} \oplus\left(s_{2}-4\right) \oplus s_{1} \quad$ for any $s_{2}-s_{1} \geq 4$.

Proof of Proposition 2.9. - The proof uses the description of quadratic differentials in terms of separatrices diagrams (see [8]) and ribbons graphs. For such diagrams, an element of $\mathcal{Q}(4 g-4)$ (respectively of $\mathcal{Q}(-1,4 g-3)$ ) is presented by a measured foliation with $4 g-4+2$ (respectively $4 g-3+2$ ) leaves emanating from the singularity. Gluing an handle of angle $s \pi$ consists topologically to glue two news sectors with an angle of $s \pi$. We thus get measured foliation with $4 g-4+2+4$ (respectively $4 g-3+2+4$ ) leaves around the new singularity. Geometrically it is easy to see that the two surgeries consisting to first glue the handle of angle $s_{1} \pi$ or to first glue the handle of angle $s_{2} \pi$ produces surfaces belonging to the same component. This is the first point of the proposition.

The two last statements are also clear in terms of diagrams. This is illustrated in Figure 2. The proposition is proven.
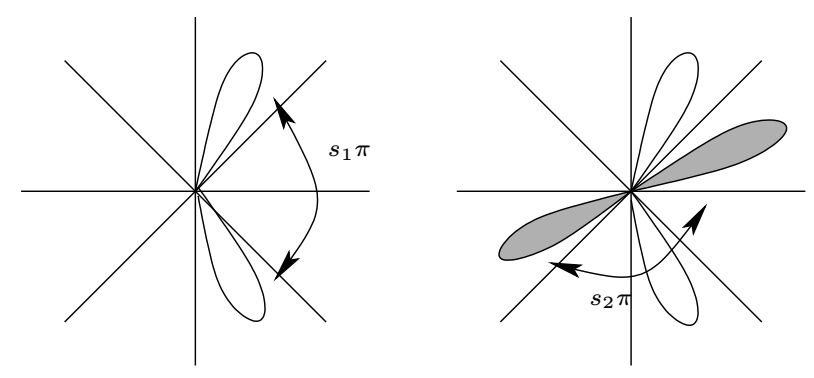

FIGURE 2. Gluing two handles attached on a singularity. Gluing first the "white" handle of angle $s_{1} \pi$ and then the "grey" handle of angle $s_{2} \pi$ is equivalent to glue first the "grey" handle of angle $\left(s_{2}-2\right) \pi$ and then the "white" handle of angle $\left(s_{1}+2\right) \pi$.

\subsection{Jenkins-Strebel surfaces}

An important class of flat metrics is given by the so-called Jenkins-Strebel differentials. We first explain these particular metrics and then we give a density result.

Let us denote by $\Gamma(q)$ the critical graph of $(\mathcal{S}, q)$ induced by the horizontal foliation, that is the union of all separatrices in the horizontal direction. It is easy to see that $\Gamma$ is compact if 
and only if the horizontal measured foliation of $q$ is completely periodic; this means that any horizontal geodesic is closed. We then call such a form a Jenkins-Strebel differential (see [19]).

Recall that locally, a stratum of the moduli space $\mathcal{Q}_{g}$ is modeled by the first cohomology group with coefficients in $\mathbb{C}$

$$
\begin{aligned}
H^{1}\left(\mathcal{S},\left\{P_{1}, \ldots, P_{n}\right\} ; \mathbb{Q}\right) \oplus i \cdot H^{1}\left(\mathcal{S},\left\{P_{1}, \ldots, P_{n}\right\} ; \mathbb{Q}\right) & \subset \\
\subset H^{1}\left(\mathcal{S},\left\{P_{1}, \ldots, P_{n}\right\} ; \mathbb{C}\right) & =H^{1}\left(\mathcal{S},\left\{P_{1}, \ldots, P_{n}\right\} ; \mathbb{R} \oplus i \mathbb{R}\right) .
\end{aligned}
$$

Taking forms such that the absolute and relative periods are in $\mathbb{Q} \oplus i \mathbb{Q}$, we get arithmetic surfaces: the orientating covering is itself a ramified covering over the standard two torus. Therefore we get that Jenkins-Strebel differentials are dense in each stratum (see [2], [5], [13], [8] and [19]).

Remark 2.10. - In [2], Douady and Hubbard proved a stronger result: the JenkinsStrebel differentials are dense on each Riemann surface, not just in a stratum.

The complement of $\Gamma(q)$ in $\mathcal{S}$ is a disjoint union of maximal periodic components for the horizontal foliation. These components are isometric to metric straight cylinders, foliated by regular horizontal leaves. A simple computation with the Euler characteristic, using the cylinders decomposition, shows that the number of the cylinders is bounded above by $3 g-3$.

In [13] Masur proved that the set of Jenkins-Strebel differentials with exactly $r$ cylinders (for any $1 \leq r \leq 3 g-3$ ) is dense inside the principal stratum $\mathcal{Q}(1, \ldots, 1)$ of genus $g$. Recently, Kontsevich and Zorich [8] have obtained a similar result. They proved that the set of JenkinsStrebel differentials with exactly one cylinder is dense inside any stratum of $\mathcal{H}_{g}$. Here we extend their proof to the case of strata of $\mathcal{Q}_{g}$.

THEOREM 2.11. - The set of quadratic differentials, such that the horizontal foliation is completely periodic and decomposes the surface in a unique straight metric cylinder, forms a dense subset of any connected component of any stratum of $\mathcal{Q}_{g}$.

Proof of Theorem 2.11. - We will only prove the existence of such surfaces on each connected component of the moduli space $\mathcal{Q}_{g}$. For the density result, we refer to the KontsevichZorich's proof. Note that in this paper, we will only need the existence result.

Let $(\mathcal{S}, q)$ be a point. Thanks to the previous discussion we may assume that the surface $(\mathcal{S}, q)$ is an arithmetic surface: its orientating double covering $\hat{\mathcal{S}}$ covers the standard torus $\mathbb{T}^{2}=\mathbb{C} / \mathbb{Z}^{2}$. The vertical foliation on $\mathcal{S}$ is completely periodic and decomposes the surface in many vertical cylinders $\mathcal{C}_{i}$.

Let us construct a closed regular curve $\gamma$ transverse to this foliation. The surface $\hat{\mathcal{S}}$ is a ramified covering $\pi: \hat{\mathcal{S}} \rightarrow \mathbb{T}^{2}$. Obviously the directional flow on $\hat{\mathcal{S}}$ projects to the directional flow on the two-torus $\mathbb{T}^{2}$. Let us consider a foliation on this torus in the direction $\theta=1 / b$ with $b$ arbitrary large. The lift of this foliation allows us to obtain a closed regular geodesic $\gamma$ on $\hat{\mathcal{S}}$, and thus a closed geodesic on $\mathcal{S}$. This leaf is transverse to the vertical foliation determined by $q$ and $\gamma$ does not contain any singularity of $q$. In addition one can choose $\gamma$ in such a way that its length with respect to the metric defined by $q$ is arbitrary large; Indeed the length of $\gamma$ is greater than $\sqrt{1+b^{2}}$.

The closed loop $\gamma$ cuts the boundaries of cylinders $\mathcal{C}_{i}$ many times (this means that $\gamma$ cuts the set of vertical saddle connections and separatrix loops of $q$ ). By construction $\partial \mathcal{C}_{i} \backslash \gamma$ is 
a disjoint union of vertical intervals. One can and do choose $\gamma$ long enough so that each component of $\partial \mathcal{C}_{i} \backslash \gamma$ contains at most one singularity of $q$. Now we will change slightly the transverse structure (in the vertical direction) to obtain a periodic horizontal foliation with only one cylinder. We will do that without changing the structure in the direction of $\gamma$.

We cut the surface along the vertical critical graph $\Gamma(q)$ of $q$ and also along $\gamma$. We obtain a finite union of parallelograms $R_{i}$. Up to the $\mathrm{SL}_{2}(\mathbb{R})$-action one may assume that $\gamma$ is vertical. The set of horizontal sides of $R_{i}$ is a part of $\gamma$ and the set of vertical sides of $R_{i}$ is a part $\Gamma(q)$. By construction in each vertical side of $R_{i}$ there is at most one singularity of $q$.

Let us construct a new foliation as follows. We conserve all horizontal parameters and we change vertical parameters in the following way: we declare that the length of any vertical side of $R_{i}$ is 1 for all $i$. In addition, if there is a singularity located on a vertical side, we declare that it is located at the middle of this side. With our above considerations, there is no contradiction. Finally we obtain a new set of parallelograms $R_{i}^{\prime}$ endowed with the natural metric $d z^{2}$.

Let $\left(\mathcal{S}^{\prime}, q^{\prime}\right)$ be the flat surface constructed from the new rectangles $R_{i}^{\prime}$ with the corresponding identifications of vertical and horizontal sides given by gluing described above. We obtain a new flat structure $q^{\prime}$ on our surface.

The surfaces $(\mathcal{S}, q)$ and $\left(\mathcal{S}^{\prime}, q^{\prime}\right)$ carry the same topology. By construction the vertical critical graphs $\Gamma(q)$ and $\Gamma\left(q^{\prime}\right)$ coincide. We just have changed absolute and relative periods of the form $q$. The subvariety of quadratic differentials sharing the same vertical foliation is connected and depends continuously on the suitable of deformations of the vertical foliation (see [5] and [21]). Thus it implies that the two points $(\mathcal{S}, q)$ and $\left(\mathcal{S}^{\prime}, q^{\prime}\right)$ belong to the same connected component. It is easy to check that in the horizontal direction on $\mathcal{S}^{\prime}$ for $q^{\prime}$ the foliation is completely periodic and decomposes the surface into a single cylinder. The theorem is proven.

\section{Generalized permutations}

In this section, we propose a natural way to encode Jenkins-Strebel differentials with one cylinder; namely we will introduce the notion of generalized permutations.

Let $(\mathcal{S}, q)$ denote a surface which is decomposed into a unique metric cylinder into the horizontal direction. Note that the holonomy of $(\mathcal{S}, q)$ is not assumed to be non-trivial.

\subsection{Combinatorics of $(\mathcal{S}, q)$}

Let $\Gamma(q)$ be the horizontal critical graph of $(\mathcal{S}, q)$. The complement $\mathcal{S} \backslash \Gamma(q)$ is a metric cylinder $\operatorname{Cyl}(\mathcal{S})$. The horizontal saddle connections are labelled by $\gamma_{1}, \ldots, \gamma_{k}$.

One can encode the sequence of saddle connections contained in the bottom and in the top of $C y l(\mathcal{S})$, and ordered following the cyclic ordering of the boundary of the cylinder, by a sequence of labels into the following manner. Each saddle connection $\gamma_{i}$ is presented twice on the boundaries of $\operatorname{Cyl}(\mathcal{S})$. Let us denote by $\gamma_{i}^{1}$ and $\gamma_{i}^{2}$ these two copies. Hence the top (respectively the bottom) of $\operatorname{Cyl}(\mathcal{S})$ is a sequence of $\gamma_{i}^{\epsilon}$ where $i \in\{1, \ldots, k\}$ and $\epsilon=1,2$. Roughly speaking a generalized permutation $\pi$ is a table with two lines encoding the sequence of labels of the saddle connections. The precise definition is the following. 
Definition 3.1. - Let $r, l$ be any non-negative integers. A generalized permutation $\pi$ (of type $(r, l))$ is an involution, without fixed points, of the set $\{1, \ldots, r, r+1, \ldots, r+l\}$.

Through this paper, we will represent a generalized permutation by a table.

EXAMPLE 3.2. - The generalized permutation (of type $(7,5)$ ) given by $\pi(1)=10, \pi(2)=$ $12, \pi(3)=5, \pi(4)=7, \pi(5)=3, \pi(6)=11, \pi(7)=4, \pi(8)=9, \pi(9)=8, \pi(10)=$ $1, \pi(11)=6, \pi(12)=2$ is represented by the table

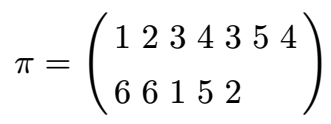

in a natural way.

The term "generalized" is justified by the fact that a classical permutation $\pi_{1}$ of the symmetric group $S_{k}$ is a generalized permutation with $l=r=k$ and

$$
\pi(i)=\left\{\begin{array}{l}
k+\pi_{1}^{-1}(i), \text { for } i \leq k \\
\pi_{1}(i-k), \text { for } i>k
\end{array}\right.
$$

In the present paper, we are interested by the quadratic differentials which are not the global square of any Abelian differential. Thus, in order to avoid "true" permutations, we require the following technical condition

$$
\text { there exist } i_{0} \leq r \text { and } j_{0} \geq r+1 \text { such that } \pi\left(i_{0}\right) \leq r \text { and } \pi\left(j_{0}\right) \geq r+1
$$

\subsection{Admissible vectors}

Let $\pi$ denote a generalized permutation of type $(r, l)$.

Definition 3.3. - We say that $\lambda \in \mathbb{R}_{+}^{r+l}$ is an admissible vector (for $\pi$ ) if

$$
\left\{\begin{array}{l}
\lambda_{i}=\lambda_{\pi(i)} \text { for all } i=1, \ldots, r+l \\
\sum_{i=1}^{r} \lambda_{i}=\sum_{j=1}^{l} \lambda_{r+j}
\end{array}\right.
$$

Note that for the "true" permutations $\pi$ the vector

$$
\left(\lambda_{1}, \ldots, \lambda_{r}, \lambda_{\pi(1)}, \ldots, \lambda_{\pi(r)}\right)
$$

is admissible for any $\lambda_{i}>0$.

$4^{\text {e }}$ SÉRIE - TOME $41-2008-\mathrm{N}^{\mathrm{o}} 1$ 


\subsection{Suspension over a generalized permutation}

Let $\pi$ be a generalized permutation and let $\lambda$ be any admissible vector for $\pi$. We denote by $w$ (width of the cylinder) the quantity

$$
w:=\sum_{i=1}^{r} \lambda_{i}=\sum_{j=1}^{l} \lambda_{r+j}
$$

Let $\mathcal{C}=[0, w] \times[0,1]$ be an Euclidean cylinder endowed with the form $d z^{2}$. Let us consider a partition of the top (respectively bottom) of $\mathcal{C}$ into horizontal intervals of length $\lambda_{i}$ for $i=1, \ldots, r$ (respectively $i=r+1, \ldots, r+l$ ). Now we identify the horizontal interval labeled $i$ with the horizontal interval $\pi(i)$ for all $i$ into the following manner. If the two intervals are presented twice on a side, we identify them by a centrally symmetry and otherwise we identify them by a translation.

The resulting space is a Riemann surface, denoted by $\mathcal{S}(\pi, \lambda)$, endowed with a natural quadratic differential $q=d z^{2}$. We call this flat surface $(\mathcal{S}, q)$ the suspension over the element $(\pi, \lambda)$.

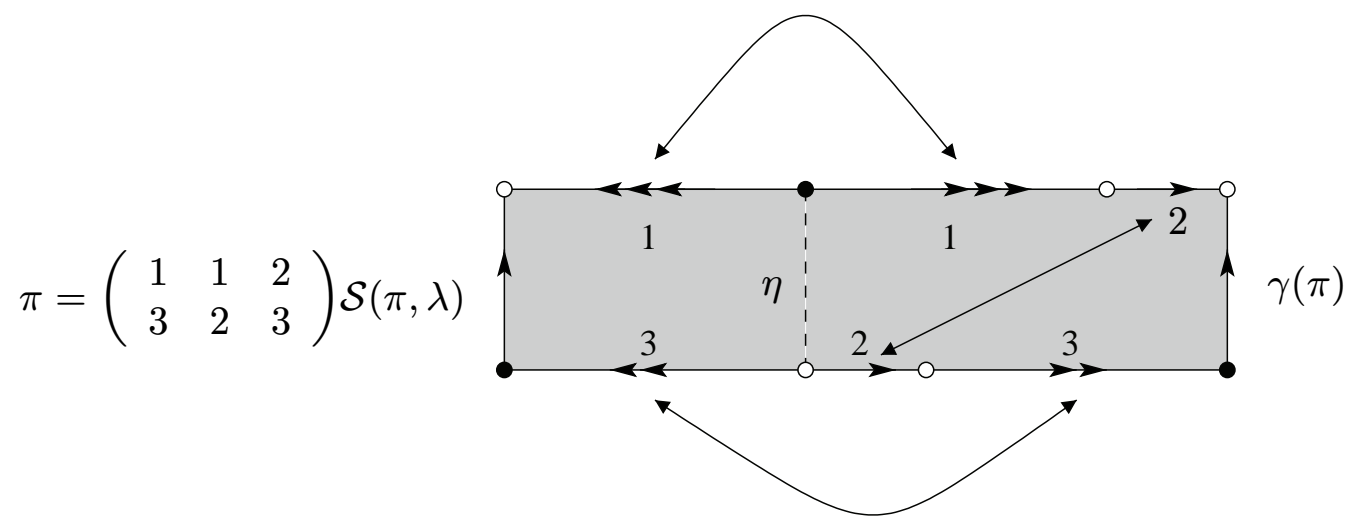

Figure 3. A suspension over a permutation $\pi$ with an appropriate admissible vector $\lambda$. The resulting point $\left(\mathcal{S}(\pi, \lambda), d z^{2}\right)$ belongs to the stratum $\mathcal{Q}(-1,-1,2)$. The black bullets correspond to the poles and the white bullets to the unique zero of the differential $q$ on $\mathcal{S}(\pi, \lambda)$.

Notation. - The surface $\mathcal{S}=\mathcal{S}(\pi, \lambda)$ decomposes in a single cylinder in the horizontal direction. By construction there exists in the vertical direction a compact separatrix on this surface. We will denote it by $\gamma(\pi) \subset \mathcal{S}$.

REMARK 3.4. $-\pi$ is a true permutation if and only if the surface $\mathcal{S}=\mathcal{S}(\pi, \lambda)$ is a translation surface.

Lemma 3.5. - The surfaces $\mathcal{S}(\pi, \lambda)$ with fixed parameter $\pi$ and arbitrary parameter $\lambda$ belong to the same connected component. 
Proof of the lemma. - The lengths $\lambda_{i}$ of the horizontal intervals correspond to the absolute and to the relative periods of the corresponding form $q$ on $\mathcal{S}$. Thus the lemma is a direct consequence of the local description of the orbifoldic structure of the strata in terms of the cohomological coordinates.

This construction implies a simple but important fact: we can encode the set of connected components using generalized permutations. Given a permutation, it determines completely the type of the singularities and hence a stratum. In addition, above lemma shows that it also determines the connected component of the stratum as well. The set of generalized permutations, for a fix stratum is obviously finite. Thus it gives an independent proof of a theorem of Veech [21].

THEOREM (Veech). - The set of connected components of a stratum $\mathcal{Q}\left(k_{1}, \ldots, k_{n}\right)$ of the moduli space $\mathcal{Q}_{g}$ of meromorphic quadratic differentials is finite.

\subsection{Cyclic order and horocyclic flow}

The class of Jenkins-Strebel quadratic differentials is stable under the horocyclic flow $h_{s}=$ $\left(\begin{array}{ll}1 & s \\ 0 & 1\end{array}\right)$. More precisely the surfaces

$$
h_{s} \cdot \mathcal{S}(\pi, \lambda)=\mathcal{S}\left(\pi^{\prime}, \lambda^{\prime}\right)
$$

are related in the following way. Elements of $\pi^{\prime}$ correspond to the elements of $\pi$ with a "rotating" of the first line and the second line. We will say that $\pi$ is equivalent to $\pi^{\prime}$ and we will note $\pi \sim \pi^{\prime}$. For example the permutation of Example 3.2 is equivalent to the following one

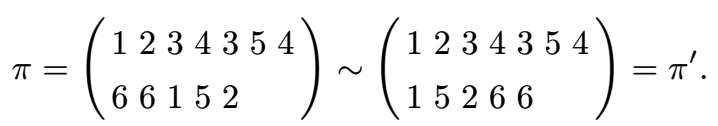

Note that this relation preserves the stratum and also the connected component.

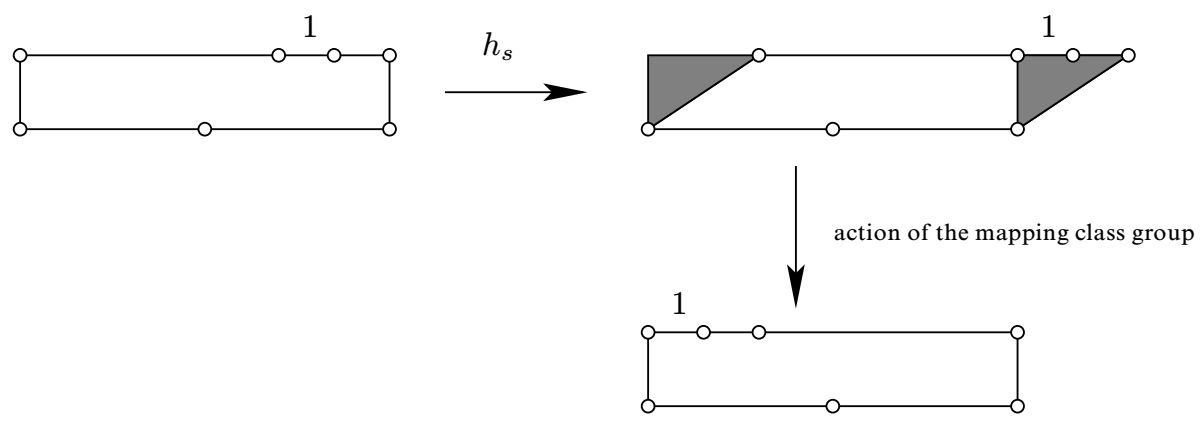

Figure 4. Action of the horocyclic flow $h_{s}$ on a flat surface.

\section{Representative elements}

In this section, we give a set of representative elements of the connected components in terms of generalized permutations (see previous Section 3). 


\subsection{Hyperelliptic connected components}

Let $r, l$ be two non-negative integers. We construct the generalized "hyperelliptic" permutation $\Pi_{1}(r, l)$ of type $(k+2, k+2)$ in the following way.

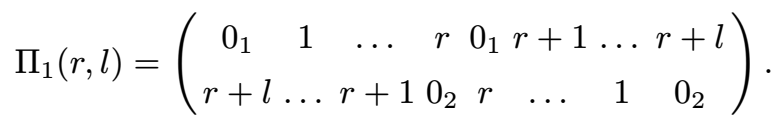

A direct computation of the type of conical angles identifies the stratum which contains surfaces $\mathcal{S}\left(\Pi_{1}(r, l), \lambda\right)$. It depends on the parity of the two integers $r$ and $l$. We easily establish the next lemma.

Lemma 4.1. - Let $(\mathcal{S}, q)$ be the flat surface given by $\mathcal{S}\left(\Pi_{1}(r, l), \lambda\right)$ for any admissible vector $\lambda$. If $r$ and $l$ are odd then $q$ has two singularities. If $r$ and $l$ have different parities then $q$ has three singularities. If $r$ and $l$ are even then $q$ has four singularities. The following table gives the type of the singularities in terms of $r$ and $l$ :

\begin{tabular}{|c|c|c|}
\hline$r$ & $l$ & stratum which contains $(\mathcal{S}, q)$ \\
\hline $2 k+1$ & $2(g-k)-3$ & $\mathcal{Q}(4 k+2,4(g-k)-6)$ \\
$2 k+2$ & $2(g-k)-3$ & $\mathcal{Q}(2 k+1,2 k+1,4(g-k)-6)$ \\
$2 k+2$ & $2(g-k)-2$ & $\mathcal{Q}(2 k+1,2 k+1,2(g-k)-3,2(g-k)-3)$ \\
\hline
\end{tabular}

According to [11], each above stratum contains a hyperelliptic connected component. We have the following lemma.

Lemma 4.2. - For any $\lambda$ and any integers $r, l$, the surface $\mathcal{S}\left(\Pi_{1}(r, l), \lambda\right)$ belongs to the hyperelliptic connected component (of the corresponding stratum).

Proof of Lemma 4.2. - Here we present the proof of the first case; that is $r$ and $l$ are odd. The other cases are similar and left to the reader. Take $r=2 k+1$ and $l=2(g-k)-3$. We consider the rectangle

$$
\mathcal{R}=\quad]-\frac{r+l}{2}-1, \frac{r+l}{2}+1[\times \quad]-1,1[
$$

Let $\tau: \mathcal{R} \rightarrow \mathcal{R}$ be the involution of $\mathcal{R}$ given by $\tau(x, y)=(-x,-y)$. The combinatorics of $\Pi_{1}$ implies that $\tau$ induces a global involution of the surface $\mathcal{S}=\mathcal{S}\left(\Pi_{1}(r, l), \lambda\right)$; we still denote it by $\tau$. By Lemma 4.1 the surface $(\mathcal{S}, q)$ belongs to the stratum $\mathcal{Q}(4 k+2,4(g-k)-6)$; therefore the Gauss-Bonnet formula implies that $\mathcal{S}$ has genus $g$.

Recall that the hyperelliptic component of this stratum is, by definition, the image of the map

$$
\begin{aligned}
\mathcal{Q}\left(2 k, 2(g-k)-4,-1^{2 g}\right) & \rightarrow \mathcal{Q}(4 k+2,4(g-k)-6) \\
\left(\mathbb{C} P(1), q_{0}\right) & \mapsto\left(\mathcal{S}_{0}, \pi^{*} q_{0}\right)
\end{aligned}
$$

where $\pi: \mathcal{S}_{0} \rightarrow \mathbb{C} P(1)$ is a double ramified covering, the ramification locus being the zeroes and poles of $q_{0}$. 
In order to prove that $(\mathcal{S}, q)$ belongs to the hyperelliptic component, we have to construct a double ramified covering $\pi: \mathcal{S} \rightarrow \mathbb{P}^{1}$ and a quadratic differential $q_{0}$ on the sphere such that $\pi^{*} q_{0}=q$. Let us count the number of fixed points of the map $\tau$ :

- there are $r+l$ fixed points of $\tau$ on the horizontal sides of $R$ located at the middle of the intervals (precisely at the middle of separatrix loops),

- there is a fixed point located at the middle of the vertical side,

- there is a fixed point at $(0,0)$,

- there are two fixed points which corresponds to the two zeroes of $q$.

Therefore the total number of fixed points of $\tau$ on $\mathcal{S}_{0}$ is

$$
r+l+1+1+2=2 k+1+2(g-k)-3+4=2 g+2 .
$$

The Riemann-Hurwitz formula implies that the genus of $\mathcal{S} /(x \sim \tau(x))$ is zero. Let us consider the projection map

$$
\pi: \mathcal{S} \rightarrow \mathcal{S} /(x \sim \tau(x)) \simeq \mathbb{C} P(1) .
$$

It is easy to check that above covering gives the desired map. Lemma 4.2 is proven.

REMARK 4.3. - The permutation $\Pi_{2}(r, l)$ of type $(2 r, 2 l)$ given by:

$$
\Pi_{2}(r, l)=\left(\begin{array}{cccccc}
1 & \ldots & r & 1 & \ldots & r \\
r+1 & \ldots & r+l & r+1 & \ldots & r+l
\end{array}\right)
$$

furnishes also a representative element for hyperelliptic connected components.

Through the proof of Theorem 7.2 on adjacency of strata, we will get a characterization of hyperelliptic components. We will obtain the following nice description.

THEOREM 4.4. - Let $(\mathcal{S}, q)$ be any point in any hyperelliptic component. Let us assume that $\mathcal{S}$ is decomposed in a unique metric cylinder for the horizontal flow. Then there exist $i \in\{1,2\}$, non-negative integers $r, l$, an admissible vector $\lambda$ and $s \in \mathbb{R}$ such that

$$
(\mathcal{S}, q)=h_{s} \cdot \mathcal{S}\left(\Pi_{i}(r, l), \lambda\right) .
$$

\subsection{Irreducible connected components}

Here we give representative elements of the sporadic components discussed in Theo-

\begin{tabular}{|c|c|}
\hline Irreducible components & Representative elements \\
\hline $\mathcal{Q}^{\operatorname{irr}}(-1,9)$ & $\left(\begin{array}{llllll}0 & 1 & 2 & 3 & 4 & 0 \\
4 & 3 & 2 & 5 & 1 & 5\end{array}\right)$ \\
\hline $\mathcal{Q}^{\text {irr }}(-1,3,6)$ & $\left(\begin{array}{lllllll}0 & 1 & 2 & 3 & 4 & 5 & 0 \\
5 & 4 & 3 & 2 & 6 & 1 & 6\end{array}\right)$ \\
\hline
\end{tabular}
rem 1.2: we will denote them by the irreducible connected components.

Definition 4.5. - The irreducible connected components are defined to be the components containing the elements $\mathcal{S}(\pi, \lambda)$ given by the next table.

$4^{\mathrm{e}}$ SÉRIE - TOME $41-2008-\mathrm{N}^{\mathrm{o}} 1$ 


\begin{tabular}{|c|c|}
\hline $\mathcal{Q}^{\text {irr }}(-1,3,3,3)$ & $\left(\begin{array}{llllllll}0 & 1 & 2 & 3 & 4 & 5 & 6 & 0 \\
6 & 5 & 3 & 2 & 7 & 4 & 1 & 7\end{array}\right)$ \\
\hline $\mathcal{Q}^{\text {irr,I }}(12)$ & $\left(\begin{array}{lllllll}1 & 2 & 3 & 4 & 2 & 5 & 6 \\
1 & 4 & 5 & 7 & 6 & 7 & 3\end{array}\right)$ \\
\hline $\mathcal{Q}^{\text {irr,II }}(12)$ & $\left(\begin{array}{lllllll}1 & 2 & 3 & 4 & 3 & 5 & 6 \\
1 & 5 & 7 & 4 & 2 & 6 & 7\end{array}\right)$ \\
\hline
\end{tabular}

\section{Dynamical properties of $\mathcal{S}(\pi, \lambda)$ versus combinatorics of $\pi$}

An important part of our proof is to find surfaces with multiplicity one saddle connection. For that we will give a combinatorial condition such that Proposition 2.2 applies.

\subsection{Irreducibility}

There exist "bad" permutations $\pi$ such that, for any $\lambda$, the saddle connection $\gamma(\pi)$ has multiplicity at least two on $\mathcal{S}(\pi, \lambda)$. More precisely the two next cases can occur.

$\forall \lambda, \exists$ saddle connection $\eta$ such that $|\eta|=\left|\gamma\left(\pi_{1}\right)\right|$ on $\mathcal{S}\left(\pi_{1}, \lambda\right)$

and

$\forall \lambda, \exists$ saddle connection $\eta$ such that $|\eta|=2\left|\gamma\left(\pi_{2}\right)\right|$ on $\mathcal{S}\left(\pi_{2}, \lambda\right)$.

The first class of "bad" permutations will lead to the notion of weak reducibility. The second class will lead to the $\operatorname{Irred}_{2}$ notion.

5.1.1. Weak irreducibility. - We say that $\pi$ (of type $(r, l)$ ) is weakly reducible if there exist $1 \leq i_{0}<r$ and $r+1 \leq j_{0}<p=r+l$ such that at least one of the following two conditions holds.

1. $\pi\left(\left\{1, \ldots, i_{0}\right\}\right)=\left\{r+1, \ldots, j_{0}\right\}$ or $\pi\left(\left\{i_{0}+1, \ldots, r\right\}\right)=\left\{r+j_{0}+1, \ldots, p\right\}$

2. each $1 \leq i \leq r$ with $1 \leq \pi(i) \leq r$ satisfies $i \leq i_{0}$ and $\pi(i)>i_{0}$. All other $i \leq i_{0}$ with $\pi(i) \geq r+1$ satisfy $\pi(i) \leq j_{0}$.

each $r+1 \leq j$ with $r+1 \leq \pi(j)$ satisfies $j \leq j_{0}$ and $\pi(j)>j_{0}$. All other $r+1 \leq j \leq j_{0}$ with $\pi(j) \leq r$ satisfy $\pi(j) \leq i_{0}$.

We will say that $\pi$ is weakly irreducible if $\pi$ is not weakly reducible.

Lemma 5.1. - Let $\mathcal{S}=\mathcal{S}(\pi, \lambda)$ be a flat surface. Then $\pi$ is weakly irreducible if and only if there exists a full Lebesgue measure set of admissible vectors $\lambda$ such that any vertical separatrix $\eta$ (different from $\gamma(\pi)$ ) on $\mathcal{S}$ satisfies $|\eta| \geq 2 \cdot|\gamma(\pi)|$.

The proof is obvious. 
Notation. - In order to clarify the situation, for a weakly reducible permutation $\pi$, we will denote by a vertical segment the position of the corresponding elements $i_{0}, j_{0}$. For instance the permutation

$$
\left(\begin{array}{lll|lll}
1 & 2 & 3 & 4 & 3 & 5 \\
6 & 1 & 2 & 6 & 5 & 4
\end{array}\right)
$$

is weakly reducible with corresponding $i_{0}=3$ and $j_{0}=9$.

5.1.2. The condition Irred $_{2}$. - This is a technical condition and we first prefer to address the relating result to this notion. The definition will then become clear.

Proposition 5.2. - Let $\mathcal{S}=\mathcal{S}(\pi, \lambda)$ be a flat surface. Let us assume that $\pi$ satisfies the condition $\operatorname{Irred}_{2}$. Then there exists a full Lebesgue measure set of admissible vectors $\lambda$ such that any vertical separatrix $\eta$ on $\mathcal{S}$ satisfies $|\eta| \neq 2 \cdot|\gamma(\pi)|$.

Definition 5.3. - We say that $\pi$ does not satisfy the condition $\operatorname{Irred}_{2}$ if there exists a (non-empty) decomposition of $\pi$ (up to exchange lines) in the following way (in terms of table)

with

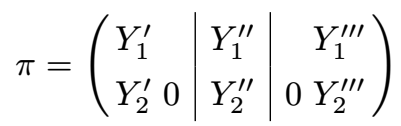

$$
\left\{\begin{array} { l } 
{ \forall i \in Y _ { 1 } ^ { \prime } \Rightarrow \pi ( i ) \in Y _ { 1 } ^ { \prime \prime \prime } \sqcup Y _ { 2 } ^ { \prime } } \\
{ \forall i \in Y _ { 1 } ^ { \prime \prime } \Rightarrow \pi ( i ) \in Y _ { 1 } ^ { \prime \prime } \sqcup Y _ { 2 } ^ { \prime \prime } }
\end{array} \quad \text { and } \left\{\begin{array}{l}
\forall j \in Y_{2}^{\prime} \Rightarrow \pi(j) \in Y_{2}^{\prime \prime \prime} \sqcup Y_{1}^{\prime} \\
\forall j \in Y_{2}^{\prime \prime} \Rightarrow \pi(j) \in Y_{2}^{\prime \prime} \sqcup Y_{1}^{\prime \prime} .
\end{array}\right.\right.
$$

EXAmple 5.4. - The permutation $\left(\begin{array}{llllll}1 & 2 & 2 & 3 & 3 & 1 \\ 0 & 0 & & \end{array}\right)$ does not satisfy the condition Irred ${ }_{2}$. Indeed, $Y_{1}^{\prime}=\{1\}, Y_{1}^{\prime \prime}=\{2,2,3,3\}, Y_{1}^{\prime \prime \prime}=\{1\}$ and $Y_{2}^{\prime}=Y_{2}^{\prime \prime}=Y_{2}^{\prime \prime \prime}=\varnothing$ is a decomposition. The permutation of Example 3.2 satisfies the condition $\operatorname{Irred}_{2}$.

Proof of Proposition 5.2. - As usual we will assume that $\gamma(\pi)$ has length 1. Recall that an admissible vector $\lambda$ satisfies a linear equation given by Equation (3.3). Let $\mathbb{R}^{r+l-1}$ be the parameters space. Let us consider the subset $E$ consisting of coordinates linearly independent over $\mathcal{Q}$. This is obviously a full Lebesgue measure set. Let us choose any $\lambda \in E$. We will prove that if there exists a vertical separatrix of length 2 , then the above decomposition of Irred occurs. $^{2}$.

Let us assume that one has found a vertical separatrix $\eta \in \mathcal{S}(\pi, \lambda)$ of length 2. A straightforward computation shows that one of the two cases presented by Figure 5 and Figure 6 has to occur.

Euclidean elementary geometry on the flat surface $\mathcal{S}$ on Figure 5 gives

$$
\sum_{i=1}^{i_{0}} \lambda_{i}=\sum_{j=1}^{j_{0}} \lambda_{j+r}-\varepsilon \quad \text { and } \quad \sum_{i=1}^{i_{1}} \lambda_{i}-\varepsilon=\sum_{j=1}^{j_{1}} \lambda_{j+r} .
$$

Adding these two formulas and recalling that $\lambda_{i_{1}}=\lambda_{j_{0}+r}$, we get

$$
2 \sum_{i=1}^{i_{0}} \lambda_{i}+\sum_{i=i_{0}+1}^{i_{1}-1} \lambda_{i}=2 \sum_{j=1}^{j_{0}-1} \lambda_{j+r}+\sum_{j=j_{0}}^{j_{1}} \lambda_{j+r} .
$$




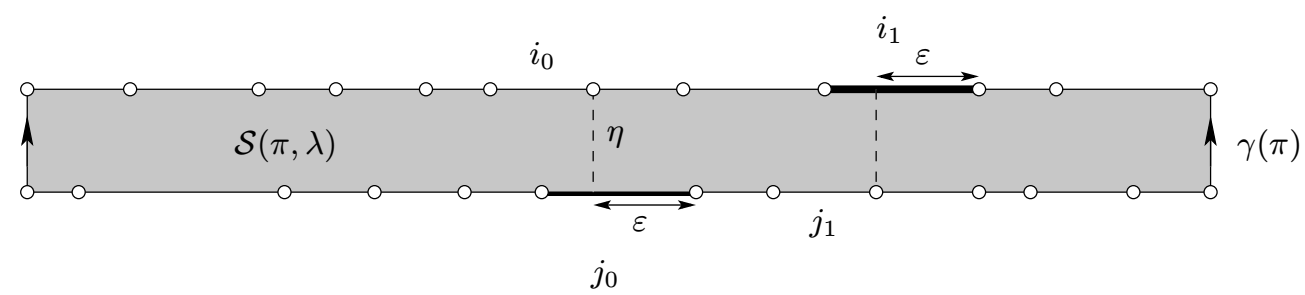

FiguRE 5. A separatrix of length 2 (here the canonical separatrix $\gamma(\pi)$ has length 1). The two corresponding horizontal intervals of lengths $\lambda_{j_{0}+r}$ and $\lambda_{i_{1}}$, labelled by $j_{0}$ and $i_{1}$, are glued by a translation.

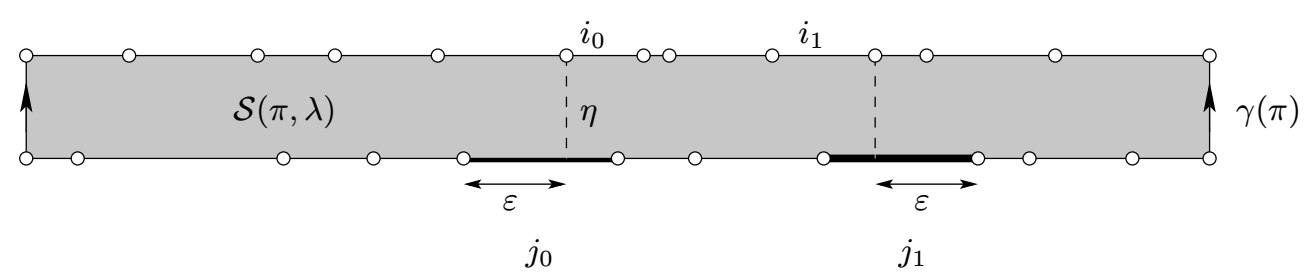

FiguRE 6. A separatrix of length 2 (here the canonical separatrix $\gamma(\pi)$ has length 1). The two corresponding horizontal intervals of lengths $\lambda_{j_{0}+r}$ and $\lambda_{j_{1}+r}$, labelled by $j_{0}$ and $j_{1}$, are glued by a central symmetry.

The same argument on Figure 6 produces similar equalities. More precisely

$$
\sum_{i=1}^{i_{0}-1} \lambda_{i}=\sum_{j=1}^{j_{0}-1} \lambda_{j+r}+\varepsilon \quad \text { and } \quad \sum_{i=1}^{i_{1}} \lambda_{i}=\sum_{j=1}^{j_{1}} \lambda_{j+r}-\varepsilon .
$$

Adding these two formulas and recalling that $\lambda_{j_{0}+r}=\lambda_{j_{1}+r}$, we get the new one:

$$
2 \sum_{i=1}^{i_{0}-1} \lambda_{i}+\sum_{i=i_{0}}^{i_{1}} \lambda_{i}=2 \sum_{j=1}^{j_{0}-1} \lambda_{j+r}+\sum_{j=j_{0}+1}^{j_{1}-1} \lambda_{j+r}+2 \lambda_{j_{0}+r} .
$$

Recall that by assumption on $\lambda$, there exists exactly one rational relation between $\lambda_{i}$. This is the following one.

$$
\sum_{i=1}^{r} \lambda_{i}=\sum_{j=1}^{l} \lambda_{r+j}
$$

It is easy to check that Equation (5.1) cannot occur: on the right part there is a term $\lambda_{j_{0}+r}$ and on the left part, the corresponding term does not appear.

Therefore only the second case arises. Comparing the coefficients of $\lambda_{i}$, and forcing the terms to cancel (using Equation (5.3)) this leads to the fact that $\pi$ does not satisfy Irred $_{2}$; with corresponding sets

$$
\begin{aligned}
& Y_{1}^{\prime}=\left(1, \ldots, i_{0}\right) \quad Y_{1}^{\prime \prime}=\left(i_{0}+1, \ldots, i_{1}-1\right) Y_{1}^{\prime \prime \prime}=\left(i_{1}, \ldots, r\right) \\
& Y_{2}^{\prime}=\left(1, \ldots, j_{0}-1\right) Y_{2}^{\prime \prime}=\left(j_{0}+1, \ldots, j_{1}-1\right) Y_{2}^{\prime \prime \prime}=\left(j_{1}+1, \ldots, l\right) .
\end{aligned}
$$


Therefore if $\lambda$ belongs to $E$ and $\pi$ satisfies $\operatorname{Irred}_{2}$, any vertical separatrix $\eta$ has length different from 2. Proposition 5.2 is proven.

\subsubsection{Irreducibility}

Definition 5.5. - We say that $\pi$ is irreducible if $\pi$ is weakly irreducible and satisfies the condition $\operatorname{Irred}_{2}$.

Proposition 5.6. - Let us consider a surface $\mathcal{S}(\pi, \lambda)$ with an irreducible permutation $\pi$. Then there exists a full Lebesgue measure set of admissible vectors $\lambda$ such that any vertical separatrix $\eta \subset \mathcal{S}(\pi, \lambda)$, different from $\gamma(\pi)$, satisfies $|\eta| \geq 3 \cdot|\gamma(\pi)|$.

Proof of Proposition 5.6. - The proof is obvious using Lemma 5.1 and Proposition 5.2: the length of any separatrix is a positive integer different from 1 and 2 for a full Lebesgue measure set.

Remark 5.7. - Proposition 5.6 is related to Keane's property. More precisely, for "true" permutations, weak irreducibility coincides with the classical definition. This means that $\pi\{1, \ldots, k\} \neq\{1, \ldots, k\}$ for any $k=1, \ldots, r-1$. The Keane's property asserts that, for irreducible permutations, for almost all $\lambda$, any separatrix $\eta \neq \gamma(\pi)$ has infinite length. See [1] for similar results in this context.

\subsection{Irreducibility and weak irreducibility}

Definition 5.8. - Let $\pi$ be a type $(r, l)$ generalized permutation. We say that $\pi$ satisfies the Condition $(*)$ if there exists only one element $i_{0} \leq r$ (respectively $j_{0} \geq r+1$ ) such that $\pi\left(i_{0}\right) \leq r$ (respectively $\left.\pi\left(j_{0}\right) \geq r+1\right)$.

We end this section by the following obvious lemma.

Lemma 5.9. - Under condition (*), weak irreducibility implies irreducibility.

\subsection{Irreducibility and "breaking up a singularity"}

Let $\pi$ be a generalized permutation and let $\lambda$ be any admissible vector. Recall (Section 3) that for a horizontal separatrix $\beta$, we denote by $\beta^{1}$ and $\beta^{2}$ the two corresponding intervals on the cylinder $\operatorname{Cyl}(\mathcal{S})$.

Proposition 5.10. - Let $\pi$ be a generalized permutation and let $\mathcal{S}=\mathcal{S}(\pi, \lambda)$ be the suspended flat surface over $(\pi, \lambda)$. If $\pi$ is irreducible then there exists a full Lebesgue measure set of $\lambda$ such that $\gamma(\pi)$ has multiplicity one.

Proof. - It follows from Proposition 5.6 and properties of homologous separatrix detailed in Proposition 2.2.

Proposition 5.11. - Let $\pi$ be a generalized permutation and let $\mathcal{S}=\mathcal{S}(\pi, \lambda)$ be the suspended flat surface over $(\pi, \lambda)$. We denote by $\beta$ and $\eta$ any two horizontal separatrices. Assume that one of the two following holds:

- $\beta^{1}$ and $\beta^{2}$ are located in two different horizontal boundaries of the cylinder $C y l(\mathcal{S})$,

- all intervals $\beta^{1}, \beta^{2}$ and $\eta^{1}, \eta^{2}$ are located in the same horizontal boundary of $C y l(\mathcal{S})$.

Then there exists a full Lebesgue measure set of $\lambda$ such that $\beta$ has multiplicity one.

$4^{\text {e }}$ SÉRIE - TOME $41-2008-\mathrm{N}^{\mathrm{o}} 1$ 
Proof of Proposition 5.11. - In the first case there is no condition on the horizontal parameter $\left|\beta^{1}\right|=\left|\beta^{2}\right|=|\beta|$ : see Equation (5.3). Thus one can and do choose the length of $\beta$ in the flat metric, arbitrary small with respect to the other length of horizontal separatrices. Therefore Proposition 2.2 applies.

In the second case there is only one linear relation on the length of $\gamma$, namely Equation (3.3). Therefore one gets:

$$
\left|\beta^{1}\right|+\left|\beta^{2}\right|+\left|\eta^{1}\right|+\left|\eta^{2}\right|+\cdots=\ldots
$$

In the left part of this equality, the terms $\beta^{i}, \eta^{i}$ survive. In particular, we can choose $\left|\beta^{1}\right|+$ $\left|\beta^{2}\right|+\left|\eta^{1}\right|+\left|\eta^{2}\right|$ arbitrary small and hence $\left|\beta^{1}\right|=\left|\beta^{2}\right|=|\beta|$ become arbitrary small with respect to the length of other horizontal separatrices. We are done.

As direct corollary, one gets the following useful result.

THEOREM 5.12. - Let us assume that either $\gamma(\pi)$ or $\beta$ (in above propositions) is a saddle connection connecting two different singularities, not two poles. Let us also assume that one of the assumptions of above Proposition 5.11 holds. Then the surface $\mathcal{S}(\pi, \lambda)$ is obtained from the surgery "breaking up a singularity" on a surface in a lower dimensional stratum for a full Lebesgue measure set of $\lambda$.

\subsection{Irreducibility and "bubbling a handle"}

Notation. - Let $\pi$ be a generalized permutation of the set $\{1, \ldots, r+l\}$ satisfying the condition $\pi(1)=r+1$. We will denote by $\hat{\pi}$ the restricted generalized permutation of the set $\{\widehat{1}, 2 \ldots, r, \widehat{r+1}, r+2, r+l\}$ (where $\hat{\imath}$ means that we have forgotten the element $i$ ). In terms of table, this gives

$$
\pi=\left(\begin{array}{ll}
0 & A \\
0 & B
\end{array}\right) \quad \text { and } \quad \hat{\pi}=\left(\begin{array}{l}
A \\
B
\end{array}\right) .
$$

Clearly, the surface $\mathcal{S}(\pi, \lambda)$, with $\pi$ as above, possesses a simple cylinder in the vertical direction (see Figure 7 and Section 2.1.1). This cylinder is filled with regular vertical closed geodesics; each boundary component is a single vertical separatrix.

Using Theorem 5.12, one deduces the following useful result.

Theorem 5.13. - Let $\mathcal{S}(\pi, \lambda) \in \mathcal{Q}_{g}(4 g-4)$ be a point. Let us assume that $\pi(1)=r+1$. If $\hat{\pi}$ is irreducible then for a full Lebesgue measure set of $\lambda$, the surface $\mathcal{S}$ is obtained from the surgery "bubbling a handle" on a surface in the stratum $\mathcal{Q}_{g-1}(4 g-8)$.

Proof of Theorem 5.13. - In the vertical direction, for any $\lambda$ the surface $\mathcal{S}$ has a simple cylinder. Let us remove it. We obtain a flat surface with boundaries. Each boundary component is a single geodesic circle: $\gamma(\pi)$ and another one, say $\beta$. By construction, they have the same length. Let us remove the singularity and glue these two geodesics segments together. We obtain a closed flat surface $\mathcal{S}^{\prime}$ of genus $g-1$. The induced quadratic differential has two singularities, say $P_{1}$ and $P_{2}$, of multiplicities $k_{1}, k_{2}$ depending on the conical angle between $\gamma(\pi)$ and $\beta$. We denote this angle by $k \pi$. By definition

$$
k_{1}=k-2 \quad \text { and } \quad k_{2}=4 g-6-k .
$$



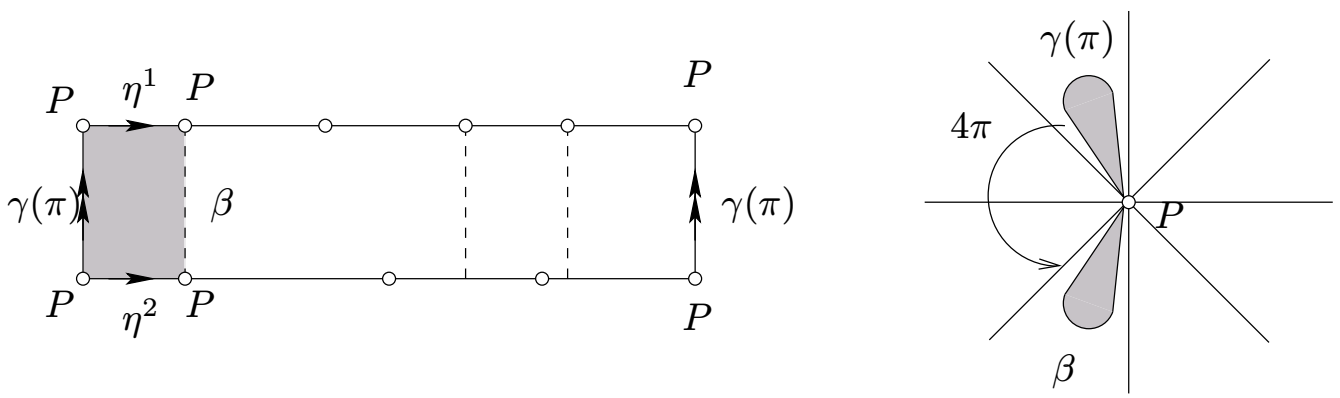

FigURE 7. On the left, the figure represents a flat surface $\mathcal{S}(\pi, \lambda)$ with $\pi$ satisfying $\pi(1)=r+1$. In the vertical direction, one can easily see that there is a simple cylinder. The boundary component of this cylinder is $\gamma(\pi) \sqcup \beta$. On the figure presented on the right, we have represented the diagram of the vertical foliation of $q=d z^{2}$. In this example, the angle of this cylinder is $4 \pi$ (or $6 \pi$ if we consider the complementary angle). If the generalized permutation $\hat{\pi}$ is irreducible, one can choose horizontal parameters so that $\gamma(\pi)$ has multiplicity one. In this case this surface is obtained from a surface in genus $g-1$, where $g=\operatorname{genus}(\mathcal{S})$, by "bubbling a handle".

By assumptions $\hat{\pi}$ is irreducible. Thus Theorem 5.12 implies that one can choose $\lambda$ in such a way that $\gamma(\pi)$ has multiplicity one. Applying Theorem 2.6 we can collapse $\gamma(\pi)$ to a point. Therefore we obtain a closed flat surface $\mathcal{S}^{\prime \prime}$ of genus $g-1$ with a unique singularity. By construction, "bubbling a handle" at the unique zero of $\mathcal{S}^{\prime \prime}$ with the appropriate angle $k \pi$, we get the surface $\mathcal{S}$. The theorem is proven.

We end this section with the following lemma.

Lemma 5.14. - The three following statements hold.

- The component $\mathcal{Q}^{\mathrm{irr}}(-1,3,6)$ is adjacent to the component $\mathcal{Q}^{\mathrm{irr}}(-1,9)$.

- The component $\mathcal{Q}^{\mathrm{irr}}(-1,3,3,3)$ is adjacent to the component $\mathcal{Q}^{\mathrm{irr}}(-1,3,6)$.

- $\mathcal{Q}^{\text {irr,I }}(12)=\mathcal{Q}(8) \oplus 2$ and $\mathcal{Q}^{\text {irr,II }}(12)=\mathcal{Q}(8) \oplus 6$.

- $\mathcal{Q}^{\text {irr }}(-1,9)=\mathcal{Q}(-1,5) \oplus 3$.

Proof of the Lemma 5.14. - We first prove the first two assertions. Let $\mathcal{S}(\pi, \lambda) \in$ $\mathcal{Q}^{\text {irr }}(-1,3,6)$ be a point with $\pi$ as in Definition 4.5. Observing the horizontal foliation, it is easy to see that there exists a multiplicity one saddle connection connecting the two zeroes. Then the first assertion deduces from Proposition 2.6. One proves the second assertion in the same manner.

Now let us concentrate on the third assertion. Let $\mathcal{S}_{1}=\mathcal{S}\left(\pi_{1}, \lambda\right), \mathcal{S}_{2}=\mathcal{S}\left(\pi_{2}, \lambda\right)$ be two representative elements of $\mathcal{Q}^{\text {irr,I }}(12), \mathcal{Q}^{\text {irr,II }}(12)$ with $\pi_{1}, \pi_{2}$ as in Definition 4.5 and $\lambda=(1, \ldots, 1)$.

We detail the first case, that is $\mathcal{S}_{1}$; the second case is similar. The vertical foliation on $\mathcal{S}_{1}$ is completely periodic and decomposes the surface in two cylinders (see Figure 8). One of the cylinders is a simple cylinder and the angle between the separatrix loops which form the boundary is $2 \pi$. Hence $\mathcal{S}_{1}=\mathcal{Q}(8) \oplus 2$. The proof of $\mathcal{S}_{2}=\mathcal{Q}(8) \oplus 6$ is similar and left to the 


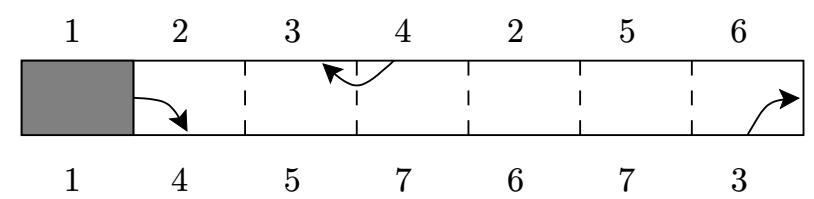

FIGURE 8. A vertical decomposition on a flat surface representing the component $\mathcal{Q}^{\text {irr,I }}(12)$.

reader.

The last statement is a direct verification left to the reader. The lemma is proven.

We now have all necessary tools to prove Main Theorem 1.1 and Main Theorem 1.2.

\section{The minimal stratum}

This section is devoted to the so-called minimal stratum $\mathcal{Q}(4 g-4)$ in case $g \geq 3$. We will prove this stratum is connected except for genus 4 . In this last case we will show there exist at most two connected components.

The proof is done by induction on the genus of the surfaces. The step of induction is given by Theorem 6.3. The initialization of the induction is reduced to the proof of the connectedness of the stratum $\mathcal{Q}_{5}(16)$ which we establish by a direct argument.

TheOREM 6.1. - The connected components of the stratum $\mathcal{Q}(4 g-4)$ are described in the following list.

- The stratum $\mathcal{Q}(8)$ is connected.

- The stratum $\mathcal{Q}(12)$ possesses at most two connected components - corresponding to $\mathcal{Q}^{\text {irr,I }}(12)$ and $\mathcal{Q}^{\text {irr,II }}(12)$.

- Any other stratum $\mathcal{Q}(4 g-4)$, in genus $g \geq 5$, is non-empty and connected.

REMARK 6.2. - The stratum $\mathcal{Q}(4 g-4)$ is empty for $g \leq 2$ (see [15]).

Zorich (see [25]) has proved that the stratum $\mathcal{Q}(12)$ is non-connected. The proof uses the Extended Rauzy classes.

\subsection{Step of induction}

THEOREM 6.3. - Let $\mathcal{C}$ be a connected component of the stratum $\mathcal{Q}(4 g-4)$ in genus $g \geq 4$. Then there exist a flat surface $(\mathcal{S}, q) \in \mathcal{Q}(4 g-8)$ and $s \in \mathbb{N}^{*}$ such that the surgery "bubbling $a$ handle" at the unique singularity of $q$ in $\mathcal{S}$ (with discrete parameter s and arbitrary continuous parameters) produces surfaces belonging to the component $\mathcal{C}$. In other words the map

$$
\begin{aligned}
\oplus: \pi_{0}\left(\mathcal{Q}_{g-1}(4 g-8)\right) & \times \mathbb{N}^{*} \rightarrow \pi_{0}\left(\mathcal{Q}_{g}(4 g-4)\right) \\
\left(\mathcal{C}^{\prime}, s\right) & \mapsto \mathcal{C}^{\prime} \oplus s
\end{aligned}
$$

is onto for any $g \geq 4$. 
REMARK 6.4. - Geometrically the previous statement is equivalent to finding a flat surface in $\mathcal{C}$ with a multiplicity one simple cylinder. In order to do that we will use Theorem 5.13. Kontsevich and Zorich have obtained a similar result in the particular case of Abelian differentials. Their proof uses Rauzy classes. Here we first give an independent (geometric) proof of their result. We then give the proof in full generality.

Hence Theorem 6.3 is equivalent to the following.

Theorem 6.5. - Let $\mathcal{C}$ be a connected component of the stratum $\mathcal{Q}(4 g-4)$ in genus $g \geq 4$. Then there exists $\mathcal{S}(\pi, \lambda) \in \mathcal{C}$ with $\hat{\pi}$ irreducible.

Proof of Theorem 6.3 for Abelian differentials. - Let $\mathcal{C} \subset \mathcal{H}(2 g-2)$ be a connected component in genus with $g \geq 2$.

Let $\pi$ be a "true" permutation in the symmetric group $S_{r}$. We will assume that the surface $\mathcal{S}(\pi, \lambda) \in \mathcal{C}$ has no marked point that is $\pi(i+1) \neq \pi(i)+1$ for all $i=1, \ldots, r$ with the "dummy" condition $\pi(r+1):=\pi(1)$. We will show that there exists a permutation $\pi_{1}$ in the class of $\pi$ for the cyclic order with $\pi_{1}(1)=1$ and such that $\hat{\pi}_{1}$ is irreducible. This will prove the theorem, indeed the surface $\mathcal{S}\left(\pi_{1}, \lambda\right) \in \mathcal{C}$.

The differential $\omega$ has a unique singularity thus one can assume, using the cyclic order, that $\pi(1)=1$. If $\hat{\pi}$ is irreducible then the theorem holds with $\pi_{1}=\pi$. Otherwise let us assume that the restricted permutation $\hat{\pi}$ is reducible. Then by definition, there exists $2 \leq i_{0}<r$ such that

$$
\pi\left(\left\{2, \ldots, i_{0}\right\}\right)=\left\{2, \ldots, i_{0}\right\} .
$$

Let us consider the following new set: $\pi\left(i_{0}+1, \ldots, r\right)=\left(A_{1} r A_{2}\right)$. With these notations, we have

$$
\left(\begin{array}{ccccccc}
1 & 2 & \ldots & i_{0} & i_{0}+1 & \ldots & r \\
1 & \ldots & \ldots & \pi\left(i_{0}\right) & A_{1} & r & A_{2}
\end{array}\right)=\pi \sim \pi_{1}=\left(\begin{array}{cccccccc}
r & 1 & 2 & \ldots & i_{0} & i_{0}+1 & \ldots & r-1 \\
r & A_{2} & 1 & \pi(2) & \ldots & \ldots & \pi\left(i_{0}\right) & A_{1}
\end{array}\right) .
$$

It is easy to see that $A_{2} \neq \varnothing$ : otherwise the corresponding surface $\mathcal{S}\left(\pi_{1}, \lambda_{1}\right)$ will possess a marked point (see above). In particular $\mathcal{S}(\pi, \lambda)$ possesses also a marked point which is a contradiction. Thus if $\hat{\pi}_{1}$ is reducible, it is easy to see that the corresponding invariant set (after re-labeling elements) $\pi_{1}\left(\left\{2, \ldots, i_{0}^{\prime}\right\}\right)=\left\{2, \ldots, i_{0}^{\prime}\right\}$ will satisfy the condition $i_{0}^{\prime} \geq$ $i_{0}+1>i_{0}$. The set $\{0, \ldots, r\}$ is finite, thus the theorem follows by repeating finitely many times this process. Theorem 6.3 for Abelian differentials is proven.

To clarify the situation, we decompose the proof in general case in several steps. We first prove the theorem in a weaker version: we add the additional assumption that there exists a surface $\mathcal{S}(\pi, \lambda) \in \mathcal{C}$ such that $\pi$ satisfies the condition $(*)$ (see Section 5.2). This corresponds to Proposition 6.6. We then prove Corollary 6.11 in order to get this additional condition. This last corollary follows from Lemma 6.9 and Lemma 6.10.

Proposition 6.6. - Let $\pi$ be a generalized permutation such that $\mathcal{S}(\pi, \lambda) \in \mathcal{Q}(4 g-4)$ with $g \geq 4$. Let us assume that $\pi$ satisfies the condition $(*)$. Then there exists $\pi_{1} \sim \pi$ such that $\hat{\pi}_{1}$ is irreducible.

We first prove the proposition for four particular classes of permutations. This corresponds to Lemma 6.8 .

$4^{\text {e }}$ SÉRIE - TOME $41-2008-\mathrm{N}^{\mathrm{o}} 1$ 
Notation. - A generalized permutation $\pi$ is an ordered partition of $X=\{0, \ldots, r+l\}$ in two ordered lists, $X=Y_{1} \sqcup Y_{2}$. In the present paper we shall always consider only those generalized permutations, for which each set $Y_{1}, Y_{2}$ contains at least one entry of multiplicity two. The permutation satisfies the condition $(*)$ so that each set $Y_{1}, Y_{2}$ contains exactly one entry of multiplicity two. Up to re-labeling, one can assume in the sequel that the two particular elements are $1 \in Y_{1}$ and $2 \in Y_{2}$. Finally, up to cyclic order, one can always put $\pi$ in the form $\pi=\left(\begin{array}{lll}0 & A & C \\ 0 & B & D\end{array}\right)$.

Note that if $\hat{\pi}$ is reducible, it involves one of the two following decomposition cases:

1. $\pi(A)=B$ or $\pi(C)=D$

2 .

For all $i \in A$, with $i \neq 1$, one has $\pi(i) \in B ; 1 \in C, \pi(1) \in C$.

For all $j \in B$, with $j \neq 2$, one has $\pi(j) \in A ; 2 \in D, \pi(2) \in D$.

In addition, we assume that this decomposition is minimal: we do not have a decomposition into smaller sets $A^{\prime}, B^{\prime}, C^{\prime}, D^{\prime}$. This condition, in case of "true" permutation, is equivalent to say that $i_{0}$ is minimal (see the proof versus Abelian differentials).

Lemma 6.7. - If $\hat{\pi}$ involves reducibility of type (1) then one can find $\pi_{1} \sim \pi$ such that $\hat{\pi}_{1}$ is irreducible.

Proof. - The proof parallels the one of Theorem 6.3 versus Abelian.

Lemma 6.8. - Let $\pi$ be a generalized permutation satisfying the condition (*). Let us also assume that one can put $\pi$ in one of the four following forms:

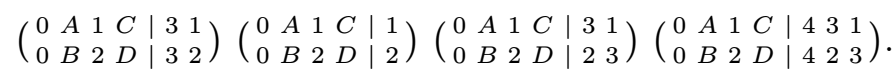

Let us assume that $\hat{\pi}$ is reducible (with corresponding marked invariant minimal sets) and $\mathcal{S}(\pi, \lambda) \in \mathcal{Q}(4 g-4)$ with $g \geq 4$. Then there exists $\pi_{1} \sim \pi$ such that $\hat{\pi}_{1}$ is irreducible.

Proof of Lemma 6.8. - Here we address the proof for the first class of permutations, the others being completely similar. By assumption on minimality of the decomposition, at least one of the two sets $C$ or $D$ is non-empty. Up to a permutation of the lines, we assume $C \neq \varnothing$. Up to re-labeling, let us denote $C:=\left(C_{1} 4\right)$ with $\pi(4) \in B \sqcup D$. Depending on the value of $\pi(4)$, we also put $B:=\left(B_{1} 4 B_{2}\right)$ or $D:=\left(D_{1} 4 D_{2}\right)$. Therefore $\pi$ is equivalent to

$$
\pi_{B}=\left(\begin{array}{cccccccc}
4 & 3 & 1 & 0 & A & 1 & C_{1} & \\
4 & B_{2} & 2 & D & 3 & 2 & 0 & B_{1}
\end{array}\right) \text { if } \pi(4) \in B \text { or } \pi_{D}=\left(\begin{array}{cccccccc}
4 & 3 & 1 & 0 & A & 1 & C_{1} & \\
4 & D_{2} & 3 & 2 & 0 & B & 2 & D_{1}
\end{array}\right) \text { if } \pi(4) \in D .
$$

This involves the two following cases:

Case 1. If $B_{2}$ or $D$ is non-empty, the restricted permutation $\hat{\pi}_{B}$ is eventually reducible but in this case it involves decomposition of type (1); thus the lemma follows from Lemma 6.7. Therefore let us assume that $B_{2}=D=\varnothing$. If $B_{1}$ is empty then the surface $\mathcal{S}(\pi, \lambda)$ belongs to the stratum $\mathcal{Q}(8)$ and hence it has genus 3 which is a contradiction. Thus up to re-labeling, one can put $B_{1}:=(B 5)$ with $\pi(5) \in A \sqcup C_{1}$. As above depending on the value of $\pi(5)$, we also put $A:=\left(A_{1} 5 A_{2}\right)$ or $C_{1}:=\left(C_{11} 5 C_{12}\right)$. Therefore $\pi$ is equivalent to

$$
\pi_{A}=\left(\begin{array}{ccccccccc}
5 & A_{2} & 1 & C_{1} & 4 & 3 & 1 & 0 & A_{1} \\
5 & 4 & 2 & 3 & 2 & 0 & B
\end{array}\right) \text { if } \pi(5) \in A \text { or } \pi_{C}=\left(\begin{array}{ccccccccc}
5 & C_{12} & 4 & 3 & 1 & 0 & A & 1 & 1 \\
5 & 4 & 2 & 3 & 2 & 0 & B
\end{array} C_{11}\right) \text { if } \pi(5) \in C_{1} .
$$

Now we easily see that each of these permutations $\hat{\pi}_{A}$ and $\hat{\pi}_{C}$ is eventually reducible but it then involves decomposition of Type (1). Thus Lemma 6.7 applies. 
Case 2. The discussion of this case is completely similar to the above one, depending on the dichotomy $\pi\left(D_{2}\right) \subseteq A$ or not. We do not give the complete details here. Lemma 6.8 is proven.

Proof of Proposition 6.6. - Let $\pi$ be a generalized permutation, satisfying condition ( $*$, such that $\mathcal{S}(\pi, \lambda) \in \mathcal{Q}(4 g-4)$ with $g \geq 4$. If $\hat{\pi}$ is reducible then, according to previous notation and Lemma 6.7, one can assume that decomposition of Type (2) arises. We have $\pi=\left(\begin{array}{lll}0 & A & C \\ 0 & B & D\end{array}\right)$ with $1 \in A$ and $\pi(1) \in C, 2 \in B$ and $\pi(2) \in D$. Let us introduce the following new notations: $C=\left(\begin{array}{lll}C_{1} & 1 & C_{2}\end{array}\right)$ and $D=\left(\begin{array}{lll}D_{1} & 2 & D_{2}\end{array}\right)$. Then we put $\pi$ in the following form $\pi=\left(\begin{array}{ll|lll}0 & A & C_{1} & 1 & C_{2} \\ 0 & B & D_{1} & 2 & D_{2}\end{array}\right)$.

Claim. - Either there exists $\pi_{1} \sim \pi$ with $\hat{\pi}_{1}$ irreducible or $\pi$ can be put in one of the two forms (with $\pi(1) \in C$ and $\pi(2) \in D$ ):

$$
\left(\begin{array}{llll}
0 & A & C & 1 \\
0 & B & D & 2
\end{array}\right) \text { or }\left(\begin{array}{lllll}
0 & A & C & 1 & 3 \\
0 & B & D & 3 & 2
\end{array}\right) \text {. }
$$

Proof of the Claim. - Obviously, if $C_{1}$ and $C_{2}$ are empty, the first form arises. Thus, by symmetry, let us assume $C_{2} \neq \varnothing$. Up to re-labeling, we put $C_{2}:=\left(C_{2} 3\right)$ with $\pi(3) \in D_{1} \sqcup$

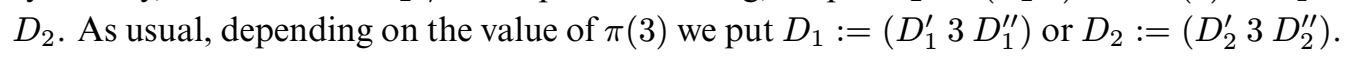
Thus, each case involves the two new permutations in the class of $\pi$ :

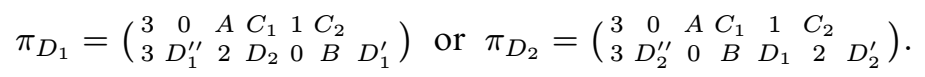

For the first permutation: If $D_{1}^{\prime \prime}=D_{2}=\varnothing$ then the permutation is of the second class of the lemma; thus we are done. Otherwise it is easy to see that the restricted permutation $\hat{\pi}_{D_{1}}$ can possibly be reducible but then the invariant set is larger than $A$ and we are done by repeating finitely many times this process.

For the second permutation: We have $D_{2}^{\prime \prime} \neq \varnothing$. As above, the restricted permutation $\hat{\pi}_{D_{2}}$ can possibly be reducible but then the new invariant set is larger than $A$ and we are also done by repeating finitely many times this process. The claim is proven.

Now we will consider the two lists $C, D$ of the claim. Each permutation of the claim produces two new classes of permutations. Here we do not give the details but the algorithm is completely similar to the one described above. Namely, the following holds:

Claim. - Either there exists $\pi_{1} \sim \pi$ with $\hat{\pi}_{1}$ irreducible or $\pi$ can be put in one of the four forms:

$$
\left(\begin{array}{lll}
0 & A & 1 \\
0 & B & 2
\end{array}\right)\left(\begin{array}{llll}
0 & A & 3 & 1 \\
0 & B & 3 & 2
\end{array}\right)\left(\begin{array}{llll}
0 & A & 3 & 1 \\
0 & B & 2 & 3
\end{array}\right)\left(\begin{array}{lllll}
0 & A & 4 & 3 & 1 \\
0 & B & 4 & 2 & 3
\end{array}\right) .
$$

Now, let us remark that each of the permutations of the previous claim is one element of the list of Lemma 6.8, and for those, we have already proved the proposition. Proposition 6.6 is proven.

Lemma 6.9. - Let $\mathcal{C}_{0}$ be a connected component of the minimal stratum $\mathcal{Q}(4 g-4)$. Then there exist two sequences of connected components

- $\mathcal{C}_{i} \subset \mathcal{Q}(4 g-4)$ for $i=0, \ldots, m(m \geq 1)$

- $\mathcal{C}^{j} \subset \mathcal{Q}\left(k_{j}, 4 g-4-k_{j}\right)$ for $j=1, \ldots, m, k_{j} \geq 1$

$4^{\text {e }}$ SÉRIE - TOME $41-2008-\mathrm{N}^{\mathrm{o}} 1$ 
and a flat surface $\mathcal{S}=\mathcal{S}(\pi, \lambda) \in \mathcal{C}_{m}$ such that:

$$
\mathcal{C}_{i} \cup \mathcal{C}_{i+1} \subset \overline{\mathcal{C}^{i+1}}, \forall i=0, \ldots, m-1 \quad \text { and } \pi \text { satisfies the condition }(*) .
$$

Proof of Lemma 6.9. - Let $\mathcal{S}(\pi, \lambda)$ be a point in $\mathcal{C}_{0}$. If $\pi$ satisfies the condition $(*)$ we are done. Otherwise let us denote $\eta_{1}, \ldots, \eta_{m}$ the set of horizontal separatrices such that $\eta_{i}^{1}$ and $\eta_{i}^{2}$ belong to the same boundary component of the cylinder $\operatorname{Cyl}(\mathcal{S})$. By assumption $m \geq 3$. Let us "break up" the unique zero in two zeroes in order to obtain a new surface, say $\mathcal{S}_{1}^{\prime}$, which belongs to a component $\mathcal{C}^{1}$. By Proposition 5.11 and the fact that $m \geq 3$, the saddle connection $\eta_{1}$ on $\mathcal{S}_{1}^{\prime}$ has multiplicity one. One can collapse it to a point to get a new surface $\mathcal{S}_{1}$ in the minimal stratum, that belongs to a component $\mathcal{C}_{1}$ (eventually different from $\mathcal{C}_{0}$ ). By construction we have

$$
C_{0} \cup C_{1} \subset \overline{\mathcal{C}^{1}}
$$

Repeating inductively this process on saddle connection $\eta_{i}$ for $i=2, \ldots, m-1$ we obtain the following diagram
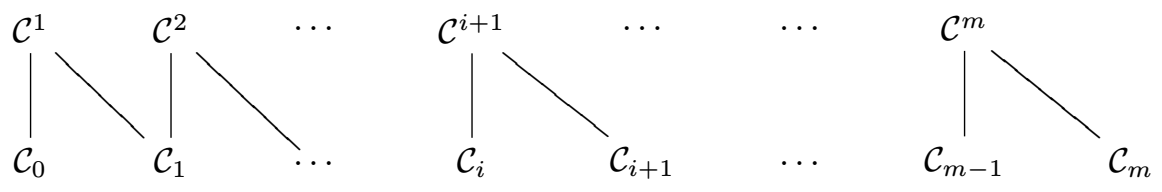

with $\mathcal{C}_{i} \cup \mathcal{C}_{i+1} \subset \overline{\mathcal{C}^{i+1}}$.

Finally by construction there exists a single pair of horizontal separatrices $\eta_{m}$ and $\eta_{m-1}$ on $\mathcal{S}_{m}\left(\pi_{m}, \lambda_{m}\right) \in \mathcal{C}_{m}$ such that $\eta_{i}^{1}$ and $\eta_{i}^{2}$ belong to the same boundary component of the cylinder $\operatorname{Cyl}(\mathcal{S})$. In other words, $\pi_{m}$ satisfies the condition $(*)$. Lemma 6.9 is proven.

Lemma 6.10. - Let $\mathcal{C}_{0} \subset \mathcal{Q}(4 g-4)$ be a connected component. Let us assume there exist two components $\mathcal{C}_{1} \subset \mathcal{Q}(4 g-4)$ and $\mathcal{C}^{1} \subset \mathcal{Q}(k, 4 g-4-k)$ such that

$$
\mathcal{C}_{0} \cup \mathcal{C}_{1} \subset \overline{\mathcal{C}^{1}}
$$

Let us also assume there exists a flat surface $(\mathcal{S}, q) \in \mathcal{C}_{1}$ with a multiplicity one simple cylinder. Then there also exists a flat surface $\left(\mathcal{S}^{\prime}, q^{\prime}\right) \in \mathcal{C}_{0}$ with a multiplicity one simple cylinder.

Proof of Lemma 6.10. - The proof is obvious using description of surfaces in terms of separatrices diagrams.

COROLlaRY 6.11. - For each connected component $\mathcal{C}$ of $\mathcal{Q}(4 g-4)$ with $g \geq 4$, there exists a surface $\mathcal{S}(\pi, \lambda) \in \mathcal{C}$ such that $\pi$ satisfies the condition $(*)$.

Proof. - It follows from Lemma 6.9 and Lemma 6.10.

Proof of Theorem 6.3. - It follows obviously from Corollary 6.11 and Proposition 6.6. 


\subsection{Connectedness of the minimal stratum}

We are ready to prove Theorem 6.1. First we directly show that $\mathcal{Q}(4 g-4)$ is connected for $g=3,5$ and has at most two components for $g=4$. We then prove the theorem inductively on $g$.

\section{LEMMA 6.12}

The stratum $\mathcal{Q}(8)$ is connected.

The stratum $\mathcal{Q}(12)$ has at most two connected components.

Proof of Lemma 6.12. - See Lemma A.1 and Lemma A.2 in the appendix.

Lemma 6.13. - The stratum $\mathcal{Q}(16)$ is connected.

Proof of Lemma 6.13. - Thanks to previous lemma let $\mathcal{C}_{0}$ be the unique connected component of the stratum $\mathcal{Q}(8)$. Let $\mathcal{C}_{1}$ be the component of $\mathcal{Q}_{5}(16)$ obtained by bubbling two handles on a surface of $\mathcal{C}_{0}$ with an angle of $2 \pi$. Namely $\mathcal{C}_{1}=\mathcal{C}_{0} \oplus 2 \oplus 2$. Recall that

$$
\mathcal{C}_{0} \oplus 2=\mathcal{Q}^{\text {irr,I }}(12) \quad \text { and } \quad \mathcal{C}_{0} \oplus 6=\mathcal{Q}^{\text {irr,II }}(12)
$$

Let $\mathcal{C}^{\prime}$ be any component of $\mathcal{Q}(16)$. By Theorem 6.3, there exists $s_{0}$ such that $\mathcal{C}^{\prime}=\mathcal{C} \oplus s_{0}$ where $\mathcal{C}$ is a component of $\mathcal{Q}(12)=\mathcal{Q}^{\text {irr,I }}(12) \cup \mathcal{Q}^{\text {irr,II }}(12)$. Using the properties of the map $\oplus$ (Proposition 2.9), we directly get the relations

$$
\mathcal{C}_{0} \oplus 2 \oplus s=\mathcal{C}_{0} \oplus 6 \oplus s=\mathcal{C}_{1} \text { for any } s=1, \ldots, 8 .
$$

Thus $\mathcal{C}^{\prime}=\mathcal{C} \oplus s_{0}=\mathcal{C}_{1}$ which proves the lemma.

Proof of Theorem 6.1. - We process by induction, initialization being given by Lemma 6.13. Let us fix $g>5$. Let us assume that $\mathcal{Q}\left(4 g^{\prime}-4\right)$ is connected for all genera $5 \leq g^{\prime}<g$. We have to show that $\mathcal{Q}(4 g-4)$ is connected. We denote by $\mathcal{C}_{g-1}=\mathcal{Q}(4(g-1)-4)$ the unique connected component of this stratum. We also define $\mathcal{C}_{g} \subseteq \mathcal{Q}(4 g-4)$ by

$$
\mathcal{C}_{g}=\mathcal{C}_{g-1} \oplus 1 \text {. }
$$

By Theorem 6.3 the map

$$
\begin{aligned}
\oplus: \pi_{0}\left(\mathcal{Q}_{g-1}(4 g-8)\right) & \times \mathbb{N}^{*} \rightarrow \pi_{0}\left(\mathcal{Q}_{g}(4 g-4)\right) \\
\left(\mathcal{C}^{\prime}, s\right) & \mapsto \mathcal{C}^{\prime} \oplus s
\end{aligned}
$$

is onto. But the stratum $\mathcal{Q}(4(g-1)-4)$ is connected and $s$ can be chosen in $\{1, \ldots, 2 g-2\}$ (up to consider the complementary angle). Thus we obtain a (onto) map

$$
\begin{gathered}
\oplus:\{1, \ldots, 2 g-2\} \rightarrow \pi_{0}\left(\mathcal{Q}_{g}(4 g-4)\right) \\
s \mapsto \mathcal{C}_{g-1} \oplus s .
\end{gathered}
$$

In order to end the proof, it remains to show that

$$
\mathcal{C}_{g}=\mathcal{C}_{g-1} \oplus s \quad \text { for any } s .
$$

Now always by Theorem 6.3 , there exists $r_{0}$ such that

$$
\mathcal{C}_{g-1}=\mathcal{C}_{g-2} \oplus r_{0} \quad \text { with } \mathcal{C}_{g-2} \subseteq \mathcal{Q}(4(g-2)-8)
$$

$4^{\text {e }}$ SÉRIE - TOME $41-2008-\mathrm{N}^{\circ} 1$ 
(this stratum is non-empty because $g-2 \geq 4)$. But recalling that the stratum $\mathcal{Q}(4(g-1)-4)$ $(g-1 \geq 5)$ is connected, we also have

$$
\mathcal{C}_{g-2} \oplus s=\mathcal{C}_{g-1} \quad \text { for any } s .
$$

Using properties of the map $\oplus$, this yields to

$$
\mathcal{C}_{g}=\mathcal{C}_{g-1} \oplus 1=\left(\mathcal{C}_{g-2} \oplus s\right) \oplus 1=\left(\mathcal{C}_{g-2} \oplus 1\right) \oplus s=\mathcal{C}_{g-1} \oplus s \quad \text { for any } s .
$$

Thus we get the desired relation (6.1). Theorem 6.1 is proven.

\section{Adjacency of the strata}

The main result we prove in this section is

Theorem 7.1. - For any $g \geq 3, n \geq 2$, let $\mathcal{C}$ be a connected component of $\mathcal{Q}_{g}\left(k_{1}, \ldots, k_{n}\right)$. Let us also assume that $\mathcal{C}$ is neither a hyperelliptic component nor an irreducible component. Then there exists a connected component $\mathcal{C}_{0} \subset \mathcal{Q}(4 g-4)$ such that $\mathcal{C}_{0} \subset \overline{\mathcal{C}}$.

We will deduce Theorem 7.1 from the following one.

THEOREM 7.2. - For any $g \geq 1$ and $n \geq 2$, let $\mathcal{C}$ be a connected component of the stratum $\mathcal{Q}_{g}\left(k_{1}, \ldots, k_{n}\right)$. Let us also assume that $\mathcal{C}$ is not of the following form:

- the hyperelliptic connected component $\mathcal{Q}^{\text {hyp }}(4(g-k)-6,4 k+2)$ or $\mathcal{Q}^{\text {hyp }}(-1,-1,4 g-2)$

- a component of $\mathcal{Q}(-1,5)$

- the irreducible component $\mathcal{Q}^{\text {irr }}(-1,9)$.

Then there exists a flat surface $(\mathcal{S}, q) \in \mathcal{Q}_{g}$ in a lower dimensional stratum and a surgery "breaking up a singularity" at a singularity of $q$ on $\mathcal{S}$ such that the resulting surface $\left(\mathcal{S}^{\prime}, q^{\prime}\right)$ belongs to $\mathcal{C}$.

Remark 7.3. - The corresponding statement for Abelian differentials is obvious. Surprisingly, in the general case we find that some connected components, in small genera, are neither hyperelliptic nor adjacent to the minimal stratum.

\subsection{Link with our main result}

Following the description of the topology of the strata of the moduli space in local coordinates (see [8]), one has

Theorem 7.4 (Kontsevich). - For any $(\mathcal{S}, q) \in \mathcal{Q}(4 g-4)$ with $g \geq 3$ there exists a small open set $U(\mathcal{S}, q)$ of $(\mathcal{S}, q)$ in the whole space $\mathcal{Q}_{g}$ such that

$$
\mathcal{Q}\left(k_{1}, \ldots, k_{n}\right) \cap U(\mathcal{S}, q)
$$

is non-empty and connected for any $\left(k_{1}, \ldots, k_{n}\right)$ with $\sum k_{i}=4 g-4$. 
Combining this result with Theorem 7.1 we get an upper bound for the number of connected components of any stratum. Let us denote by $r_{g}$ the number of connected components of the minimal stratum $\mathcal{Q}(4 g-4)$. Then for any $g \geq 3$, Theorem 7.1 and Theorem 7.4 together give

$$
\begin{aligned}
& 1 \leq \#\left\{\begin{array}{l}
\text { components of a stratum which neither contains } \\
\text { a hyperelliptic component nor an exceptional component }
\end{array}\right\} \quad \leq r_{g} \\
& 2 \leq \#\{\text { components of a stratum which contains a hyperelliptic component }\} \leq r_{g}+1 \\
& 1 \leq \#\{\text { components of a stratum which contains an exceptional component }\} \leq r_{g}+1
\end{aligned}
$$

In Section 6 we have proved that $r_{g}=1$ for $g \geq 5$ which implies main Theorem 1.1.

\subsection{Strategy of the proof of Theorem 7.2}

Let $\mathcal{C}$ be a connected component of the stratum $\mathcal{Q}_{g}\left(k_{1}, \ldots, k_{n}\right)$ with $g \geq 1$ and $n \geq 2$. According to Theorem 2.6 one has to construct a surface $(\mathcal{S}, q) \in \mathcal{C}$ with a multiplicity one separatrix between two different singularities, not two poles. In order to do that we will use the criterion given by Proposition 5.11 and Proposition 5.10.

Let $(\mathcal{S}(\pi, \lambda), q)$ be a point in $\mathcal{C}$. Either Proposition 5.11 applies and then we are done or assumptions of the proposition do not hold. In this last case we get some restrictions on the combinatorics of $\pi$.

Now let us consider the vertical foliation on $(\mathcal{S}(\pi, \lambda), q)$. As above either Proposition 5.10 applies and we are done or assumptions of the proposition again do not hold. In this last case we also get new restrictions on the combinatorics of $\pi$. In particular a simple computation shows that the permutation $\pi$ is completely determined. It corresponds to a "hyperelliptic" or "irreducible" permutation. These two cases are avoid; indeed the component $\mathcal{C}$ is neither hyperelliptic nor irreducible.

The proof is decomposed into several cases. Recall that $n$ denotes the number of singularities. First we consider the general case $n \geq 4$. We then prove the case $n=3$. Finally we conclude with the holomorphic case $n=2$ and the meromorphic case $n=2$, that is the stratum $\mathcal{Q}(-1,4 g-3)$.

REMARK 7.5. - The corresponding statement of Theorem 7.2 versus Abelian differentials is trivial. Indeed Proposition 5.11 applies directly (all transitions functions are given by translations).

\subsection{Proof of Theorem 7.2 in case $n \geq 4$}

Let $\mathcal{C}$ be a connected component and $\mathcal{S}(\pi, \lambda) \in \mathcal{C}$. We denote the horizontal cylinder of $\mathcal{S}$ by $\operatorname{Cyl}(\mathcal{S})$. The boundaries components of $C y l(\mathcal{S})$ are denoted by $I$ and $J$. One associates to each saddle connection $\eta$ two intervals $\eta^{1}, \eta^{2}$ on $I \sqcup J$.

Claim 1. - There exists a saddle connection $\alpha$ between a zero $P_{1}$ and another singularity $P_{2}$.

$4^{\text {e }}$ SÉRIE - TOME $41-2008-\mathrm{N}^{\mathrm{o}} 1$ 
Proof of the claim. - The assumption $g \geq 1$ implies there exists at least a zero $P_{1}$. If there exists a saddle connection attached to this zero we are done. Otherwise any separatrix emanating from $P_{1}$ is actually a separatrix loop.

Exchanging the role of $I$ and $J$ if necessary, the previous assertion means that any saddle connection located in $I$ is a separatrix loop emanating from $P_{1}$ (see Figure 9a).

We claim there exists a separatrix attached to a zero, say $P_{1}^{\prime} \neq P_{1}$, located on $J$. Otherwise it will mean that any separatrix in $J$ is attached to a pole. The only possibility to obtain such a configuration is presented in Figure $9 b$. In particular the stratum is $\mathcal{Q}(k,-1,-1)$ contradicting the assumption $n \geq 4$. If there exists no saddle connection attached to $P_{1}^{\prime}$ then any

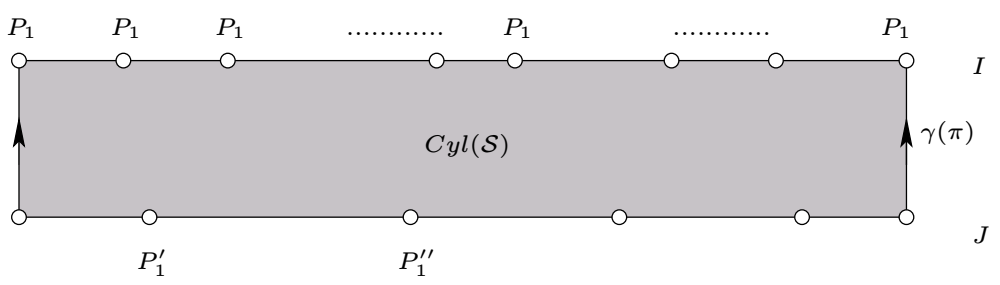

(a)

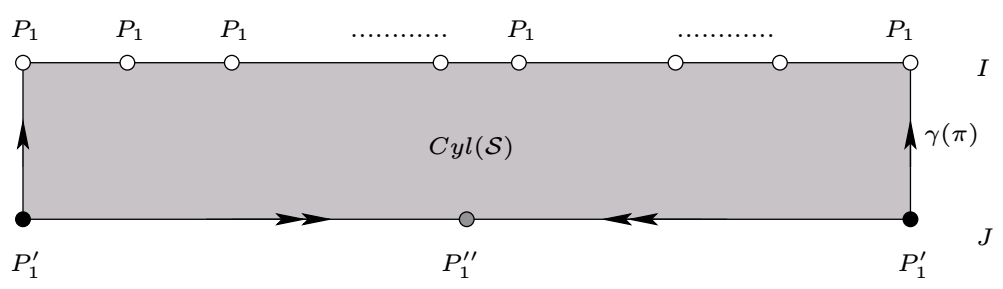

(b)

Figure 9.

separatrix located on $J$ is a separatrix loop emanating from $P_{1}^{\prime}$. It implies that the surface has only two zeroes. This is again a contradiction and the claim is proven.

Let $\alpha$ be a saddle connection between the zero $P_{1}$ and the singularity $P_{2}$ (we assume that $\alpha^{1} \subset I$ ). If $\alpha^{2} \subset J$ then Proposition 5.11 implies that $\alpha$ has multiplicity one and the theorem is proven. Hence let us assume that

$$
\alpha^{1}, \alpha^{2} \subset I
$$

(we refer to Figure 10a). 


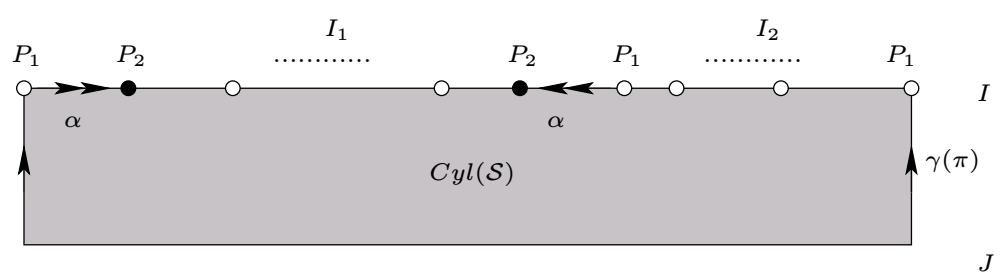

(a)

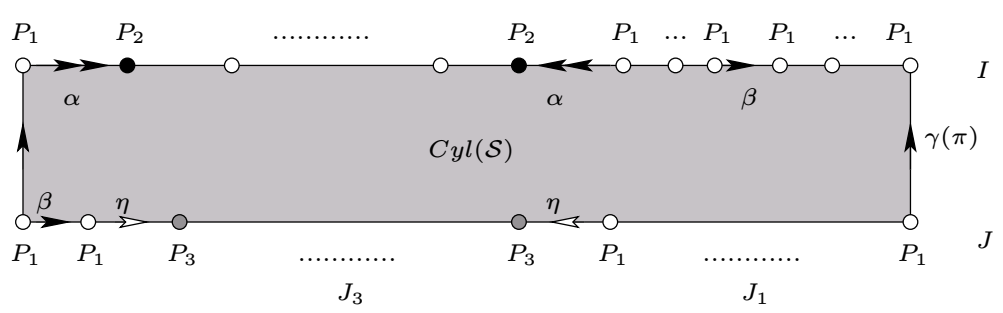

(b)

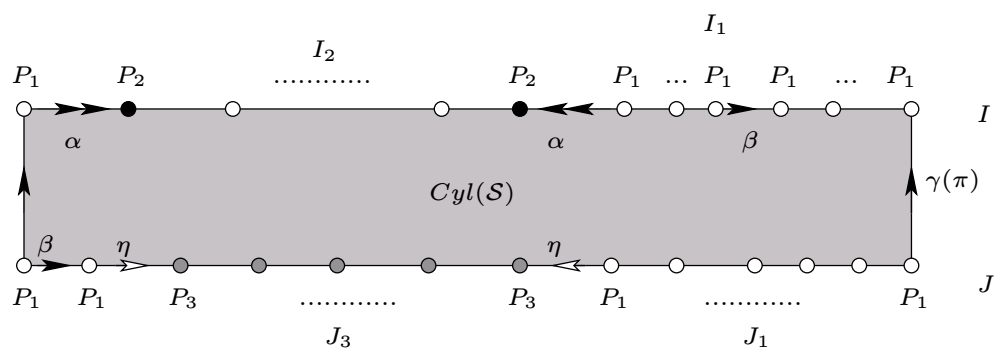

(c)

Figure 10 .

The complement of $\alpha^{1}, \alpha^{2}$ in $I$ has two connected components. Following the figure, we will refer to these two components as $I_{1}, I_{2}$. The interval $I_{i}$ is a union of separatrices.

Claim 2. - Either the theorem is proved or any separatrix $\tau$, with $\tau^{1} \subset I_{i}$, is a separatrix loop attached to $P_{i}$. In addition $\tau^{2} \subset J$ (eventually $I_{2}=\varnothing$ ).

Proof of the claim. - Observe that either there exists a saddle connection $\tau$ between two singularities (not two poles) with $\tau^{1} \subset I_{1}$ or any separatrix of $I_{1}$ is a separatrix loop attached to $P_{1}$. The first case is the conclusion of the theorem. Thus let us consider the second case. 
Let $\tau$ be a separatrix with $\tau^{1} \subset I_{1}$. Then $\tau^{2} \subset I \sqcup J$. Recalling that $\alpha^{1}, \alpha^{2} \subset I$ and applying Proposition 5.11 we get that if $\tau^{2} \subset I$ then $\alpha$ has multiplicity one and therefore we again get the conclusions of the theorem. Otherwise $\tau^{2} \subset J$ giving the second conclusion of the claim.

Repeating the same argument for separatrices $\tau$ with $\tau^{1} \subset I_{2}$, we get the claim.

Let us assume that the second conclusion of Claim 2 holds. The singularity $P_{1}$ is a zero; hence there exists a separatrix loop $\beta$ attached to $P_{1}$ with $\alpha_{1} \subset I_{1}$. Thanks to Claim 2, $\alpha^{2} \subset J$ (see Figure 10b). In particular there exists a saddle connection $\eta$ between $P_{1}$ and a singularity $P_{3} \neq P_{1}$ (possibly $P_{3}=P_{2}$ ) such that $\eta^{1} \subset J$. If $\eta^{2} \subset I$ then thanks to Proposition 5.11, $\eta$ has multiplicity one and the theorem is proved. Thus one can and do assume $\eta^{2} \subset J$. The complement of $\eta^{1}, \eta^{2}$ in $J$ has two connected components. Following the figure, we will refer to these two components as $J_{1}, J_{3}$. The interval $J_{i}$ is a union of separatrices.

CLAIM 3. - Either the theorem is proved or any separatrix $\tau$, with $\tau^{1} \subset J_{i}$, is a separatrix loop attached to $P_{i}$. In addition $\tau^{2} \subset I$.

Proof of the claim. - The proof parallels the previous one (see Claim 2).

Finally we can summarize above claims. Either the theorem is proved or $\mathcal{S}$ can be put in the form prescribed by Figure 10c. The last case implies that $\mathcal{S}$ has at most three singularities: $P_{1}, P_{2}, P$ contradicting $n \geq 4$. Therefore Theorem 7.2 in case $n \geq 4$ is proven.

\subsection{Proof of Theorem 7.2 in case $n=3$}

The proof is similar to the case $n \geq 4$ with a refinement. We first prove the following lemma.

Lemma 7.6. - Let $\mathcal{C}$ be a connected component of $\mathcal{Q}_{g}\left(k_{1}, k_{2}, k_{3}\right)$ with $g \geq 1$. Then there exists a flat surface $(\mathcal{S}(\pi, \lambda), q) \in \mathcal{C}$ such that one of the two following holds.

- $(\mathcal{S}(\pi, \lambda), q)$ has a multiplicity one saddle connection between two different singularities (not two poles),

- the singularity pattern is exactly $\left(k_{1}, k_{2}, k_{3}\right)=(-1,-1,4 g-2)$ and the combinatorics of $\pi$ has the form

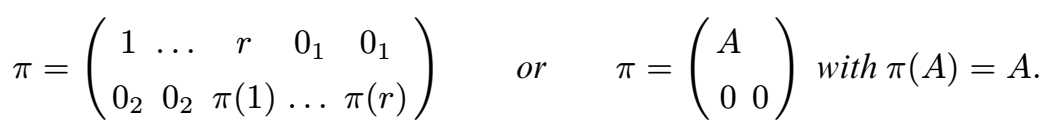

Proof of Lemma 7.6. - Let $\mathcal{S}(\pi, \lambda)$ be a point in $\mathcal{C}$. Imitating the proof of Claim 1 one gets that either there exists a saddle connection $\alpha$ between a zero and another singularity or one can put the surface $\mathcal{S}$ in the form prescribed by Figure $9 \mathrm{~b}$. The last case produces a surface $\mathcal{S}(\pi, \lambda)$ belonging to the stratum $\mathcal{Q}(k,-1,-1)$ with $\pi=\left(\begin{array}{cc}A & 0 \\ 0 & 0\end{array}\right)$ and $\pi(A)=A$; and so the lemma is proven.

Therefore let us assume that $\mathcal{S}$ possesses a saddle connection $\alpha$ between a zero and another singularity. One can extend Claim 2 and Claim 3 to the case $n=3$ which leads to the following dichotomy. Either $\mathcal{S}$ possesses a multiplicity one saddle connection, and so the lemma is proven, or $\mathcal{S}$ can be put in the form prescribed by Figure 10c. 
Finally one can assume the following situation: Any separatrix $\tau$ belonging to $I_{1}$ or $J_{1}$ is a separatrix loop attached to $P_{1}$. In addition $\tau^{1} \subset I_{1}$ and $\tau^{2} \subset J_{1}$. Moreover $n=3$ thus $P_{3} \neq P_{2}$.

If $P_{2}$ is not a pole then there exists a separatrix loop $\tau$ attached to $P_{2}$ with $\tau^{1} \subset I_{2}$. If $\tau^{2} \subset J$ then $\tau^{2} \subset J_{3}$ implying $P_{2}=P_{3}$ which is contradiction. Therefore $\tau^{2} \subset I$. Now Proposition 5.11 implies that $\alpha$ has multiplicity one (indeed $\alpha^{1}, \alpha^{2} \subset I$ ) and so the lemma is proven. The same proof holds if $P_{3}$ is not a pole.

Therefore let us assume that $P_{2}$ and $P_{3}$ are two poles. Then the stratum containing $\mathcal{S}$ is $\mathcal{Q}(k,-1,-1)$ and the permutation $\pi$ has the combinatorics $\left(\begin{array}{ccccc}1 & \ldots & r & 0_{1} & 0_{1} \\ 0_{2} & 0_{2} & \pi(1) & \ldots & \pi(r)\end{array}\right)$. The lemma is proven.

Therefore in order to prove Theorem 7.2 in case $n=3$ we have to analyse the combinatorics of two permutations given in Lemma 7.6.

Lemma. - Let $\pi$ be the permutation $\left(\begin{array}{cccccc}2 & \ldots & r & 0_{1} & 0_{1} & 1 \\ 0_{2} & \pi(1) & \pi(2) & \ldots & \pi(r) & 0_{2}\end{array}\right)$. Let us assume that $\pi$ is different (up to the cyclic order) from $\Pi_{1}(r, 0)$. Then there exists $\pi^{\prime} \sim \pi$ such that $\pi^{\prime}$ is irreducible and $\gamma\left(\pi^{\prime}\right)$ is a saddle connection between the zero and a pole.

Proof of the lemma. - First of all, let us remark that the separatrix $\gamma(\pi)$ is actually a saddle connection. Let us also remark that $\pi$ satisfies the condition (*), therefore by Lemma 5.9, weak irreducibility implies irreducibility for any permutation in the class of $\pi$.

The above generalized permutation $\pi$ is reducible if and only if $\pi(r)=1$. One observes that

$$
\pi=\left(\begin{array}{cccccc}
2 & \ldots & r & 0_{1} & 0_{1} & 1 \\
0_{2} & \pi(1) & \pi(2) & \ldots & \pi(r) & 0_{2}
\end{array}\right) \sim \pi^{\prime}=\left(\begin{array}{ccccccc}
3 & \ldots & r & 0_{1} & 0_{1} & 1 & 2 \\
0_{2} & \pi(1) & \pi(2) & \ldots & \pi(r-1) & 1 & 0_{2}
\end{array}\right) .
$$

It is easy to check that $\pi^{\prime}$ is irreducible if and only if $\pi(r-1)=2$.

Therefore repeating finitely many times this process, one shows that either there exists an irreducible permutation $\pi^{\prime}$ in the class of $\pi$ or

$$
\pi(i)=r-i+1 \quad \text { for all } i=1, \ldots r .
$$

The last equations mean that $\pi \sim \Pi_{1}(r, 0)$ which is a contradiction. The lemma is proven.

Lemma. - Let $\pi=\left(\begin{array}{cc}A & \\ 0 & 0\end{array}\right)$ with $\pi(A)=A$. Assume $\pi$ is different (up to the cyclic order) from $\Pi_{2}(r, 1)$. Then there exists $\pi^{\prime} \sim \pi$ such that $\pi^{\prime}$ is irreducible and $\gamma\left(\pi^{\prime}\right)$ is a saddle connection between the zero and a pole.

Proof. - It is easy to see that for each permutation $\pi^{\prime}$ in the class of $\pi$, the separatrix $\gamma\left(\pi^{\prime}\right)$ is actually a saddle connection between the zero and a pole. If $\pi$ is weakly reducible then by definition, we have $\pi=\left(\begin{array}{cc}B & C \\ 0 & 0\end{array}\right)$ with $\pi(B)=C$. Let us decompose the two lists $B, C$ in the

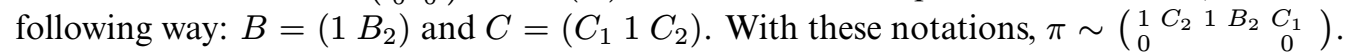
This permutation is weakly reducible if and only if $C_{1}=\varnothing$.

One can repeat finitely many times this process, with $B:=B_{2}$ and $C:=C_{2}$ for the new sets, to get the following statement. Either there exists a weakly irreducible permutation $\pi^{\prime} \sim \pi$ or $B=C=\left(\begin{array}{llll}1 & 2 & \ldots r\end{array}\right)$. The last case means that $\pi=\Pi_{2}(r, 1)$ which is a contradiction. 
Therefore we have proved that there exists a weakly irreducible $\pi^{\prime} \sim \pi$. From this permutation it is easy to check that there exists $\pi^{\prime \prime} \sim \pi^{\prime}$ irreducible. Hence $\pi^{\prime \prime} \sim \pi$ and the lemma is proven.

Now we are ready to prove Theorem 7.2 in case $n=3$. Let $\mathcal{C}$ be any component of any stratum $\mathcal{Q}_{g}\left(k_{1}, k_{2}, k_{3}\right)$. Applying Lemma 7.6 combining with the two above lemmas one gets the following dichotomy. There exits $\mathcal{S} \in \mathcal{C}$ such that either $\mathcal{S}$ possesses a multiplicity one saddle connection between two singularities (not two poles), and so the theorem is proved, or $\mathcal{S}=\mathcal{S}(\pi, \lambda)$ with $\pi=\Pi_{1}(r, 0)$ or $\pi=\Pi_{2}(r, 1)$. These last two permutations correspond to hyperelliptic permutations and in particular it implies that $\mathcal{C}$ is itself a hyperelliptic component of $\mathcal{Q}(-1,-1,4 g-2)$ which is a contradiction. The theorem is proven.

\subsection{Proof of Theorem 7.2 in the holomorphic case $n=2$}

We first prove the following lemma.

Lemma 7.7. - For any $k_{1}, k_{2}>0$ and $g \geq 2$, let $\mathcal{C}$ be a connected component of the stratum $\mathcal{Q}_{g}\left(k_{1}, k_{2}\right)$. Then there exists $(\mathcal{S}(\pi, \lambda), q) \in \mathcal{C}$ a flat surface such that one of the two following holds.

- $(\mathcal{S}(\pi, \lambda), q)$ has a multiplicity one saddle connection between the two zeroes.

- The combinatorics of $\pi$ has the form

$$
\begin{gathered}
\pi=\left(\begin{array}{cccccccc}
0_{1} & 1 & \ldots & r & 0_{1} & r+1 & \ldots & r+l \\
0_{2} & \sigma_{1}(1) & \ldots & \sigma_{1}(r) & 0_{2} & \sigma_{2}(r+1) & \ldots & \sigma_{2}(r+l)
\end{array}\right) \text { or } \pi=\left(\begin{array}{c}
A \\
B
\end{array}\right) \text { with } \pi(A)=A, \pi(B)=B \\
\text { where } \sigma_{1}, \sigma_{2} \text { are "true" permutations of the sets }\{1, \ldots, r\} \text { and }\{r+1, \ldots, r+l\} .
\end{gathered}
$$

Proof of Lemma 7.7. - The proof is similar to the one of Lemma 7.7, it parallels the one of Claim 1, Claim 2 and Claim 3.

Therefore in order to prove Theorem 7.2 in the holomorphic case $n=2$ we have to analyse the two permutations of Lemma 7.7.

Lemma. - Let $\pi$ be the permutation $\left(\begin{array}{cccccccccc}0 & 1 & \ldots & r & 0_{1} & r+1 & \ldots & r+l \\ \sigma_{1}(r) & 0_{2} & \sigma_{2}(r+1) & \ldots & \sigma_{2}(r+l) & 0_{2} & \sigma_{1}(1) & \ldots & \sigma_{1}(r-1)\end{array}\right)$. Let us assume that $\pi \neq \Pi_{1}(r, l)$. Then there exists $\pi^{\prime} \sim \pi$ such that $\pi^{\prime}$ is irreducible and $\gamma\left(\pi^{\prime}\right)$ is a saddle connection between the two zeroes.

Proof of the lemma. - Let us first remark that the separatrix $\gamma(\pi)$ on $\mathcal{S}(\pi, \lambda)$ is actually a saddle connection. Note also that $\pi$ satisfies Condition $(*)$; thus the permutation $\pi$ is irreducible if and only if $\pi$ is weakly irreducible.

One checks directly that $\pi$ is reducible if and only if $\sigma_{1}(r)=1$. In this case one can put $\pi$ in the form

$$
\begin{aligned}
\pi=\left(\begin{array}{ccccccccc}
0_{1} & 1 & \ldots & r & 0_{1} & r+1 & \ldots & r+l \\
\sigma_{1}(r) & 0_{2} & \sigma_{2}(r+1) & \ldots & \sigma_{2}(r+l) & 0_{2} & \sigma_{1}(1) & \ldots & \sigma_{1}(r-1)
\end{array}\right) & \sim \pi^{\prime}= \\
& =\left(\begin{array}{cccccccccc}
0_{1} & 1 & 2 & \ldots & r & 0_{1} & r+1 & \ldots & r+l & \\
\sigma_{1}(r-1) & 1 & 0_{2} & \sigma_{2}(r+1) & \ldots & \sigma_{2}(r+l) & 0_{2} & \sigma_{1}(1) & \ldots & \sigma_{1}(r-2)
\end{array}\right) .
\end{aligned}
$$

Repeating this process to $\pi^{\prime}$ this yields to the following dichotomy. Either there exists an irreducible permutation $\pi^{\prime}$ with $\pi^{\prime} \sim \pi$, and so the lemma is proven, or

$$
\left\{\begin{array}{l}
\sigma_{1}(i)=r-i+1 \quad \text { for } i=1, \ldots, r \\
\sigma_{2}(j)=2 r+l-j+1 \text { for } j=r+1, \ldots, r+l
\end{array}\right.
$$


These last equalities mean that $\pi=\Pi_{1}(r, l)$ which is a contradiction. The lemma is proven.

Lemma. - Let $\pi=\left(\begin{array}{c}A \\ B\end{array}\right)$ with $\pi(A)=A, \pi(B)=B$. Let us also assume that $\pi \neq \Pi_{2}(r, l)$. Then there exists $\pi^{\prime} \sim \pi$ such that $\pi^{\prime}$ is irreducible and $\gamma\left(\pi^{\prime}\right)$ is a saddle connection between the two zeroes.

Proof. - The proof is completely similar to the previous one and left to the reader.

We are now ready to prove Theorem 7.2 in the holomorphic case $n=2$. Let $\mathcal{C}$ be any component of any stratum $\mathcal{Q}_{g}\left(k_{1}, k_{2}\right)$. Applying Lemma 7.7 combining with the two above lemma one gets the following dichotomy. There exits $\mathcal{S} \in \mathcal{C}$ such that either $\mathcal{S}$ possesses a multiplicity one saddle connection between the two zeroes, and so the theorem is proved, or $\mathcal{S}=\mathcal{S}(\pi, \lambda)$ with $\pi=\Pi_{1}(r, l)$ or $\pi=\Pi_{2}(r, l)$. These last two permutations correspond to hyperelliptic permutations and in particular it implies that $\mathcal{C}$ is itself a hyperelliptic component of $\mathcal{Q}(4(g-k)-6,4 k+2)$ (where $r=2 k+1$ and $l=2(g-k)-3$ ) which is a contradiction. The theorem is proven.

\subsection{Proof of Theorem 7.2 in case $\mathcal{Q}(-1,4 g-3)$}

The proof is decomposed in two steps. We recall that a simple cylinder is a metric cylinder with boundary components consisting of two single homologous separatrices. A simple cylinder has multiplicity one if its boundary has multiplicity one.

Proposition 7.8. - Let $\mathcal{C} \subset \mathcal{Q}(-1,4 g-3)$ with $g \geq 3$. Then there exists $\mathcal{S}(\pi, \lambda) \in \mathcal{C} a$ flat surface such that one of the two followings holds.

- $\mathcal{S}$ has a multiplicity one saddle connection between the zero and the pole.

- $\mathcal{S}$ has a multiplicity one simple cylinder (i.e. $\mathcal{C}=\mathcal{C}^{\prime} \oplus s$ with $s \in\{1, \ldots, 2 g\}$ and $\mathcal{C}^{\prime} \subset$ $\mathcal{Q}(-1,4(g-1)-3)$ is a connected component $)$.

Proposition 7.9. - The following assertions hold.

1. For any $s \in\{1,2,4\}$, there exists a flat surface $(\mathcal{S}, q) \in \mathcal{Q}(-1,5) \oplus s$ with a multiplicity one saddle connection.

2. Any connected component of $\mathcal{Q}(-1,13)$ possesses a flat surface with a multiplicity one saddle connection.

3. If $(\mathcal{S}, q)$ has a multiplicity one saddle connection then so has $(\mathcal{S}, q) \oplus s$ (by bubbling a handle).

We postpone the proof of the second proposition to the appendix (see Proposition A.4). Let us first show how these two propositions give the theorem and then let us prove the first proposition.

Proof of Theorem 7.2. - It is done by induction on $g$. The theorem is already proven in case $g=4$ (Proposition 7.9, (2)). Let $g>4$ be any integer and let $\mathcal{C} \subset \mathcal{Q}(-1,4 g-3$ ) be a connected component. One has to prove that $\mathcal{C}$ possesses a surface with a multiplicity one saddle connection.

Thanks to Proposition 7.8, either $\mathcal{C}$ possesses a surface with a multiplicity one saddle connection, and then the theorem is proved, or $\mathcal{C}$ contains a surface with a multiplicity one simple

$4^{\mathrm{e}}$ SÉRIE - TOME $41-2008-\mathrm{N}^{\mathrm{o}} 1$ 
cylinder. In the last eventuality we have $\mathcal{C}=\mathcal{C}^{\prime} \oplus s$ where $\mathcal{C}^{\prime} \subset \mathcal{Q}(-1,4(g-1)-3)=$ $\mathcal{Q}\left(-1,4 g^{\prime}-3\right)$ is a connected component. We have $4 \leq g^{\prime}<g$ thus by assumption $\mathcal{C}^{\prime}$ contains a surface $\mathcal{S}^{\prime}$ with a multiplicity one saddle connection. Then we conclude by Proposition 7.9, (3): $\mathcal{S}^{\prime} \oplus s \in \mathcal{C}$ is the required surface and the theorem is proven.

It remains to prove the theorem for the genus $g=3$ case. Again from Proposition 7.8, if $\mathcal{C} \subset \mathcal{Q}(-1,9)$ has no surface with multiplicity one saddle connection then $\mathcal{C}$ has the form $\mathcal{Q}(-1,5) \oplus s$ with $s=1,2,3,4$. Moreover Lemma 5.14, (5.14) implies $\mathcal{Q}(-1,9)^{\mathrm{irr}}=$ $\mathcal{Q}(-1,5) \oplus 3$. Therefore the theorem follows from Proposition 7.9, (1) which examines cases $s=1,2,4$. The theorem is proven.

Proof of Proposition 7.8. - Let $\mathcal{C} \subset \mathcal{Q}(-1,4 g-3)$ be a connected component with $g \geq 3$ and $\mathcal{S}(\pi, \lambda) \in \mathcal{C}$. The surface $\mathcal{S}$ has a pole and a unique zero $P$. Hence one can put $\mathcal{S}$ in the form prescribed by Figure 11.

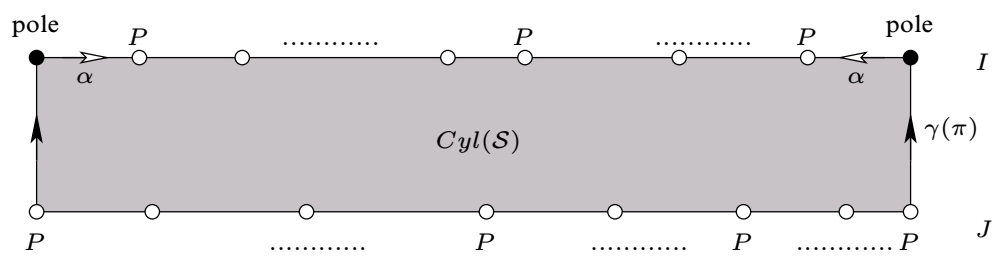

FIGURE 11. A flat surface with a unique zero and a unique pole.

Let $\alpha$ be the horizontal saddle connection between the pole and the zero.

Claim. - Either $\alpha$ has multiplicity one or $\mathcal{S}(\pi, \lambda)=\mathcal{S}\left(\pi^{\prime}, \lambda^{\prime}\right)$ with $\pi^{\prime}=\left(\begin{array}{lllll}0 & 1 & \ldots & r & 0\end{array}\right)$ and $\pi^{\prime}(\{1, \ldots, r\}) \subset A$.

Proof of the claim. - Apply Proposition 5.11.

Therefore thanks to the above claim let us assume that $\mathcal{S}(\pi, \lambda) \in \mathcal{C}$ where $\pi$ is a permutation given by previous claim. We define $\sigma \sim \pi$ by $\sigma=\left(\begin{array}{cccc}1 & 2 \\ 1 & \ldots\end{array} \quad r \quad 00\right)$ and $\sigma(\{1, \ldots, r\}) \subset A$. Note also that the vertical direction on $\mathcal{S}(\sigma, \lambda)$ decomposes the surface in (at least) one simple cylinder (see also Figure 7). The reducibility of $\hat{\sigma}$ involves one of the two following possible decompositions

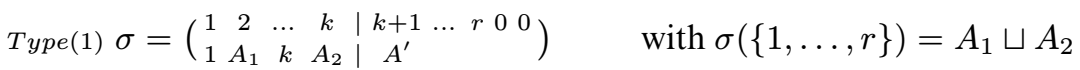

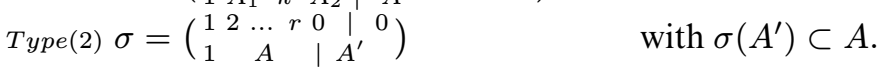

ClaIm. - If $\hat{\sigma}$ involves reducibility of Type (1) then there exists $\sigma_{1} \sim \sigma$ such that $\hat{\sigma}_{1}$ is irreducible.

Proof of the claim. - The proof parallels the one of Theorem 6.3 versus Abelian differentials (Section 6.1).

For reducible permutations $\sigma$ of Type (2), let us denote $A=\left(A_{1} k A_{2}\right)$ with $\sigma\left(A_{2}\right) \subseteq A^{\prime}$ and $2 \leq k \leq r$ (eventually $A_{2}=\varnothing$ ). 
CLAIm. - If $\hat{\sigma}$ involves reducibility of Type (2) then either there exists $\sigma_{1} \sim \sigma$ such that $\hat{\sigma}_{1}$ is irreducible or $k=r$.

Proof of the claim. - With above notations, if $k \neq r$ then one has

$$
\sigma=\left(\begin{array}{cccccccc}
1 & 2 & \ldots & k & \ldots & r & 0 & 0 \\
1 & A_{1} & k & A_{2} & & A^{\prime}
\end{array}\right) \sim \sigma_{1}=\left(\begin{array}{cccccccccc}
k & k+1 & \ldots & r & 0 & 0 & 1 & 2 & \ldots & k-1 \\
k & A_{2} & A^{\prime} & 1 & A_{1} & & & & &
\end{array}\right) .
$$

If $\hat{\sigma}_{1}$ is irreducible then the claim is proven. Otherwise it is easy to see that $\hat{\sigma}_{1}$ involves reducibility of Type (1). Thus previous claim applies.

Finally the last case we have to consider is the following one: $\hat{\sigma}$ is reducible with decomposition of Type (2) and $k=r$. Reporting these data in the permutation $\pi$ one gets $\pi=$ $\left(\begin{array}{ccccc}0 & 1 & \ldots & \multicolumn{1}{c}{r} & 0 \\ r & A_{2} & A^{\prime} & 1 & A_{1}\end{array}\right)$ with $\pi\left(A_{2}\right) \subseteq A^{\prime}$.

Claim. - The vertical separatrix $\gamma(\pi)$ on $\mathcal{S}(\pi, \lambda)$ is a saddle connection. Moreover the above permutation $\pi$ is reducible if and only if

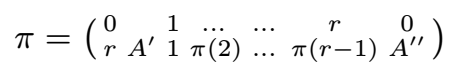

with $A^{\prime \prime}=\pi\left(A^{\prime}\right)$.

Proof of the claim. - Let $\pi=\left(\begin{array}{ccccc}0 & 1 & \ldots & \multicolumn{1}{c}{} & 0 \\ r & A_{2} & A^{\prime} & 1 & A_{1}\end{array}\right)$ with $\pi\left(A_{2}\right) \subseteq A^{\prime}$. A direct observation shows that if $\pi$ is reducible then $A_{2}=\varnothing$. The same approach shows that if $\pi$ is reducible then $A_{1}=\left(\pi(2) \ldots \pi(r-1) A^{\prime \prime}\right)$ with $\pi\left(A^{\prime}\right)=A^{\prime \prime}$. The claim is proven.

The conclusion of previous claims is the following one. We have proved that $\mathcal{C}$ contains a surface $\mathcal{S}(\pi, \lambda)$ such that either $\mathcal{S}$ satisfies the conclusion of the proposition or

$$
\pi=\left(\begin{array}{ccccccc}
0 & 1 & \ldots & \ldots & r & 0 \\
r & A^{\prime} & 1 & \pi(2) & \ldots & \pi(r-1) & A^{\prime \prime}
\end{array}\right) \quad \text { and } \quad \pi\left(A^{\prime}\right)=A^{\prime \prime} .
$$

Then we have to study the last two cases $r \geq 2$ and $r=1$. This corresponds to the next two lemmas which end the proof of Proposition 7.8.

LeMma. - Let $r \geq 2$ be any integer. Let $\pi=\left(\begin{array}{ccccccc}0 & A^{\prime} & \ldots & \ldots & r & 0 \\ r & A^{\prime} & \pi(2) & \cdots & \pi(r-1) & A^{\prime \prime}\end{array}\right)$ be a permutation with $\pi\left(A^{\prime}\right)=A^{\prime \prime}$. We also assume that $\mathcal{S}(\pi, \lambda) \in \mathcal{Q}(-1,4 g-3)$ with $g \geq 3$.

Then there exists a permutation $\pi^{\prime} \sim \pi$ such that $\mathcal{S}\left(\pi^{\prime}, \lambda\right)$ has a multiplicity one saddle connection.

Proof of the lemma. - Let us assume that $r \geq 3$, then $r-1 \neq 1$. Let us introduce some notations to clarify the situation. Let $C$ stand for $(\pi(2) \ldots \pi(r-1))$. One decomposes $C$ in the following way: $C=\left(C_{1} r-1 C_{2}\right)$. Equipped with these notations: $\pi=$ $\left(\begin{array}{ccccccc}0 & 1 & \cdots & r-1 & r & 0 \\ r & A^{\prime} & 1 & C_{1} & r-1 & C_{2} & A^{\prime \prime}\end{array}\right) \sim \sigma=\left(\begin{array}{ccccccc}0 & 1 & \ldots & \ldots & r-1 & r & 0 \\ r-1 & C_{2} & A^{\prime \prime} & r & A^{\prime} & 1 & C_{1}\end{array}\right)$. A direct observation shows that $\sigma$ is irreducible and $\gamma(\sigma)$ is a saddle connection. The lemma then follows from Theorem 5.12.

Now let us assume that $r=2$. The list $A^{\prime \prime}$ is non-empty. Let us denote $A^{\prime \prime}=(3 B)$ with

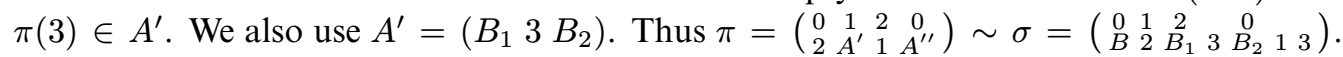
This permutation is reducible if and only if $B=B_{1}=B_{2}=\varnothing$. This case consists of $\pi=$ $\left(\begin{array}{llll}0 & 1 & 2 & 0 \\ 2 & 3 & 1 & 3\end{array}\right)$ and then $\mathcal{S}(\pi, \lambda) \in \mathcal{Q}(-1,5)$ contradicting $g \geq 3$. Hence $\sigma$ is irreducible and the lemma again follows from Theorem 5.12.

Next lemma studies the $r=1$ case.

$4^{\mathrm{e}}$ SÉRIE - TOME $41-2008-\mathrm{N}^{\mathrm{o}} 1$ 
Lemma. - Let $\pi=\left(\begin{array}{ccc}0 & 1 & 0 \\ A^{\prime} & 1 & A^{\prime \prime}\end{array}\right)$ be a permutation with $\pi\left(A^{\prime}\right)=A^{\prime \prime}$. Then one of the following assertions holds.

- There exists $\pi^{\prime} \sim \pi$ such that $\mathcal{S}\left(\pi^{\prime}, \lambda\right)$ has a multiplicity one saddle connection.

- The combinatorics of $\pi$ is given by $A^{\prime}=A^{\prime \prime}=(23 \ldots l)$. Moreover there exist $\pi^{\prime} \sim \pi$ and an admissible vector $\lambda_{0}$ such that $\mathcal{S}\left(\pi^{\prime}, \lambda_{0}\right)$ has a multiplicity one simple cylinder.

Proof of the lemma. - Let $\pi$ be the permutation $\pi=\left(\begin{array}{ccc}0 & 1 & 0 \\ A^{\prime} & 1 & A^{\prime \prime}\end{array}\right)$. The list $A^{\prime}$ is non-empty thus let us introduce $A^{\prime}=\left(2 A_{2}^{\prime}\right)$ with $\pi(2) \in A^{\prime \prime}$. Hence we can also introduce lists $A_{1}^{\prime \prime}, A_{2}^{\prime \prime}$

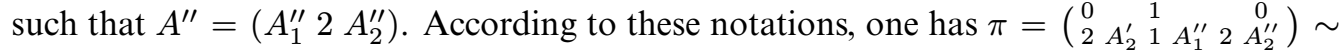
$\pi^{\prime}=\left(\begin{array}{lllll}0 & A_{2}^{\prime \prime} & 1 & & 0 \\ 2 & A_{2}^{\prime} & 2 & A_{2}^{\prime \prime} & A_{1}^{\prime \prime}\end{array}\right)$. A straightforward computation shows that the permutation $\pi^{\prime}$ is reducible if and only if $A_{1}^{\prime \prime}=\varnothing$. Thus, repeating inductively this process with $A^{\prime}:=A_{2}^{\prime}$ and $A^{\prime \prime}:=A_{2}^{\prime \prime}$, we get either $\mathcal{S}\left(\pi^{\prime}, \lambda\right)$ has a multiplicity one saddle connection or

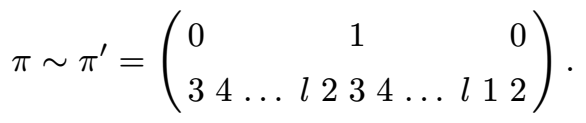

In order to finish the proof of the lemma, we have to present an admissible vector $\lambda_{0}$ such that the vertical foliation on $\mathcal{S}\left(\pi^{\prime}, \lambda_{0}\right)$ is completely periodic and decomposes the surface with at least a multiplicity one simple cylinder. Let us consider the admissible vector (for $\pi^{\prime}$ ):

$$
\lambda_{0}=((l-1) \alpha ; \alpha ;(l-1) \alpha ; \underbrace{\alpha ; \ldots ; \alpha}_{2 l-1 \text { times }}) \quad \text { for any } \alpha \in \mathbb{R}^{+} .
$$

It is easy to see that the vertical foliation on $\mathcal{S}\left(\pi^{\prime}, \lambda_{0}\right)$ decomposes the surface in $g-1$ cylinders (see Figure 12). One checks that the vertical cylinder corresponding to the horizontal interval numbered $l$ is a multiplicity one simple cylinder (see the figure). In Figure 12, we present a complete description for the surface given by the case $r=5$ (i.e. $g=3$ ). This completes the proof of the lemma.

Now we can deduce from Theorem 7.2 our main result, namely Theorem 7.1.

\subsection{Proof of Theorem 7.1}

We first establish

Proposition 7.10 (Reformulation of Theorem 7.2 in the holomorphic case $n=2$ )

$\mathcal{C} \subseteq \mathcal{Q}\left(k_{1}, k_{2}\right), k_{i}>0$, is hyperelliptic $\Longleftrightarrow$

$\forall(\mathcal{S}, q) \in \mathcal{C}$, any saddle connection on $\mathcal{S}$ has multiplicity at least 2.

Through the proof of Theorem 7.2 in case $n=3$ and $n=4$ one gets a similar characterization of hyperelliptic components (compare with Lemma 2.4).

Proposition 7.11. - Hyperelliptic components of strata with 3 and 4 singularities are characterized by the following:

1. $\mathcal{C} \subseteq \mathcal{Q}\left(k_{1}, k_{2}, k_{3}\right)$ is hyperelliptic $\Longleftrightarrow \exists i_{0} \neq i_{1} \in\{1,2,3\}, \forall(\mathcal{S}, q) \in \mathcal{C}$,

any saddle connection on $\mathcal{S}$ between $P_{k_{i}}$ and $P_{k_{j}}$, with $i \in\left\{i_{0}, i_{1}\right\}$ and $j \notin\left\{i_{0}, i_{1}\right\}$, has multiplicity at least 2 . 

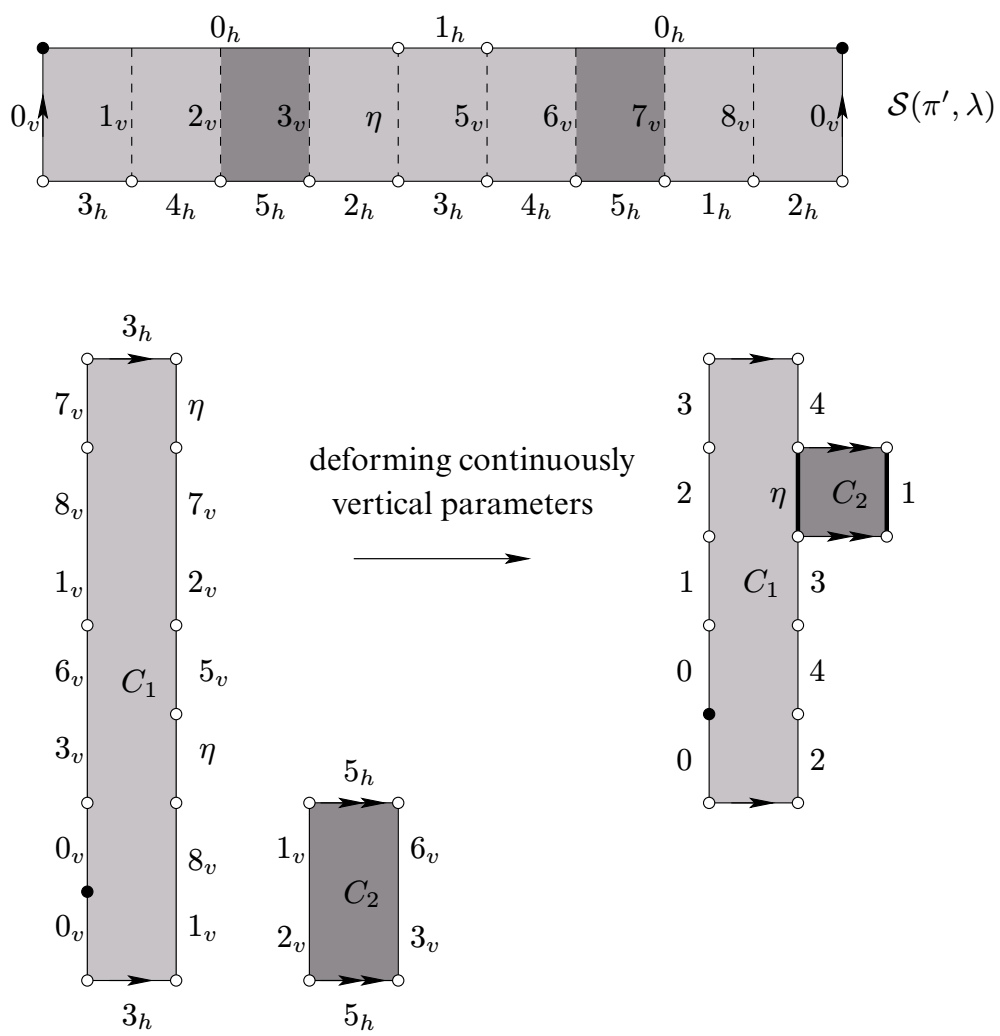

FIgURE 12. A flat surface $\mathcal{S}^{\prime}=\mathcal{S}\left(\pi^{\prime} \lambda_{0}\right)$ suspended over the permutation $\pi^{\prime}$ and the admissible vector $\lambda_{0}$ (see Equation (7.1). The vertical foliation decomposes $\mathcal{S}^{\prime}$ in two cylinders $C_{1}$ and $C_{2}$. The cylinder $C_{2}$ is a simple cylinder: the conical angle of its boundary $\eta$ is $\pi$. Moreover the length of $\eta$ can be chosen arbitrary small with respect to other vertical parameters: $C_{2}$ has multiplicity one.

2. $\mathcal{C} \subseteq \mathcal{Q}\left(k_{1}, k_{2}, k_{3}, k_{4}\right)$ is hyperelliptic $\Longleftrightarrow \exists i_{0} \neq i_{1} \in\{1,2,3,4\}, \forall(\mathcal{S}, q) \in \mathcal{C}$, any saddle connection on $\mathcal{S}$ between $P_{k_{i}}$ and $P_{k_{j}}$, with $i \in\left\{i_{0}, i_{1}\right\}$ and $j \notin\left\{i_{0}, i_{1}\right\}$, has multiplicity at least 2 .

Proposition 7.12. - Let $\mathcal{C}$ be a connected component of $\mathcal{Q}\left(k_{1}, \ldots, k_{n}\right)$ with $n \geq 5$. Then for each $i \neq j$, there exists a flat surface $\mathcal{S} \in \mathcal{C}$ with a multiplicity one saddle connection between two singularities $P_{k_{i}}, P_{k_{j}} \in \mathcal{S}$ of multiplicities $k_{i}$ and $k_{j}$.

Proof of Theorem 7.1. - We discuss the theorem following the different values of $n$ (the number of singularities). First let us note that if we prove the theorem in case $n=2,3,4,5$ then the theorem follows for any $n \geq 6$ because the locus of hyperelliptic and irreducible components is located on strata with 2,3 and 4 singularities.

The theorem in case $n=2$ corresponds to Theorem 7.2 which is already proved.

Thus let us assume that $n=3$. Let $\mathcal{C} \subseteq \mathcal{Q}\left(k_{1}, k_{2}, k_{3}\right)$ be a non-hyperelliptic component. Recall that all $k_{i}$ are non-zero. Up to permuting the $k_{i}$ 's, one can assume $k_{1} \leq k_{2} \leq k_{3}$. 
Proposition 7.11 with $i_{0}=1, i_{1}=2$, gives a flat surface $(\mathcal{S}, q) \in \mathcal{C}$ with a multiplicity one saddle connection between $P_{k_{3}} \in \mathcal{S}$ and $P_{k_{i}} \in \mathcal{S}$ for $i \in\{1,2\}$. In other terms there exists a component $\mathcal{C}^{\prime}$ of the strata $\mathcal{Q}\left(k_{1}+k_{3}, k_{2}\right) \sqcup \mathcal{Q}\left(k_{2}+k_{3}, k_{1}\right)$ such that $\mathcal{C}^{\prime} \subset \overline{\mathcal{C}}$. There are three possibilities: $\mathcal{C}^{\prime}$ is hyperelliptic, irreducible or "regular" (neither hyperelliptic nor irreducible).

If $\mathcal{C}^{\prime}$ is regular then Theorem 7.2 leads to the result. Indeed one can find $\mathcal{C}_{0} \subseteq \mathcal{Q}(4 g-4)$ a component with $\mathcal{C}_{0} \subset \overline{\mathcal{C}^{\prime}}$. But $\overline{\mathcal{C}^{\prime}} \subset \overline{\mathcal{C}}$ therefore $\mathcal{C}_{0} \subset \overline{\mathcal{C}}$ and we are done.

Assume that $\mathcal{C}^{\prime}$ is hyperelliptic. Applying Proposition B.1, one can connect the hyperelliptic component $\mathcal{C}^{\prime}$ to another non-hyperelliptic component $\mathcal{C}^{\prime \prime}$ of $\mathcal{Q}\left(k_{i}+k_{3}, k_{j}\right)$ passing through the stratum $\mathcal{Q}\left(k_{1}, k_{2}, k_{3}\right)$. Therefore there exists a component $\mathcal{C}_{2} \subseteq \mathcal{Q}\left(k_{1}, k_{2}, k_{3}\right)$ such that $\mathcal{C}^{\prime} \sqcup \mathcal{C}^{\prime \prime} \subset \overline{\mathcal{C}_{2}}$. Thus $\mathcal{C}^{\prime} \subset \overline{\mathcal{C}} \sqcup \overline{\mathcal{C}_{2}}$. The assumption $0 \leq k_{1} \leq k_{2} \leq k_{3}$ implies $k_{i}+k_{3} \neq k_{j}$; thus Corollary 2.7 implies $\mathcal{C}_{2}=\mathcal{C}$. Therefore $\mathcal{C}$ is adjacent to a non-hyperelliptic component of $\mathcal{Q}\left(k_{i}+k_{3}, k_{j}\right)$ and we are done again.

Finally let us assume that $\mathcal{C}^{\prime}$ is irreducible; that is $\mathcal{C}^{\prime}=\mathcal{Q}^{\text {irr }}(-1,9)$. Recall that the component $\mathcal{C} \neq \mathcal{Q}^{\operatorname{irr}}(-1,3,6)$, therefore the theorem follows from Proposition B.2 and Corollary 2.7 .

The proof for cases $n=4,5$ is similar to the above discussion. Theorem 7.1 is proven.

\section{Proof of Main Theorem 1.1 and Main Theorem 1.2}

Proof of Main Theorem 1.1. - As mentioned in Section 7.1, Main Theorem 1.1 follows from Theorem 7.1 on the adjacency of the strata and Theorem 6.1 on the classification of the minimal strata.

Proof of Main Theorem 1.2. - Theorem 1.2 is already proved for the genus 0 case (see Proposition 1.6). The genus 3 case is also proved (see Theorem 7.1 and Section 7.1).

Thus let us consider genera 1 and 2 cases. One can first prove, by a direct argument on finitely many strata, Theorem 1.2 for $g=1,2$ and $n \leq 5$ cases. Indeed the number of such a strata of $\mathcal{Q}_{2}$ and of $\mathcal{Q}_{1}$ with $n=2,3,4,5$ is finite. We can check, using extended Rauzy classes, that the theorem holds. In particular we get that any stratum of $\mathcal{Q}_{2}$ with $n=5$ is connected and any stratum of $\mathcal{Q}_{1}$ with $n=5$ is also connected.

Now let us prove that any stratum of $\mathcal{Q}_{1}$ with $n \geq 6$ is also connected. Let $\mathcal{C}_{1}, \mathcal{C}_{2}$ be two components of $\mathcal{Q}\left(k_{1}, \ldots, k_{6}\right)$. Let us assume that $k_{1} \leq \cdots \leq k_{6}$. Then $k_{5}+k_{6} \neq k_{i}$ for any $i=1, \ldots, 4$. By Proposition 7.12 there exist two components $\mathcal{C}_{0}$ and $\mathcal{C}_{0}^{\prime}$ of $\mathcal{Q}\left(k_{1}, \ldots, k_{4}, k_{5}+\right.$ $k_{6}$ ) with $\mathcal{C}_{0} \subset \overline{\mathcal{C}_{1}}$ and $\mathcal{C}_{0}^{\prime} \subset \overline{\mathcal{C}_{2}}$. Any stratum of $\mathcal{Q}_{1}$ with $n=5$ being connected, one has $\mathcal{C}_{0}=\mathcal{C}_{0}^{\prime}$, hence $\mathcal{C}_{0} \subset \overline{\mathcal{C}_{1}} \cup \overline{\mathcal{C}_{2}}$. Therefore Corollary 2.7 applies and $\mathcal{C}_{1}=\mathcal{C}_{2}$; so that any stratum of $\mathcal{Q}_{1}$ with $n=6$ is connected. The above argument extends to any stratum of $\mathcal{Q}_{1}$ with $n \geq 6$. Theorem 1.2 in case $g=1$ is therefore proven. The same argument holds for the genus 2 case.

We now prove Theorem 1.2 in the case $g=4$. We have already proved that any component of a stratum of $\mathcal{Q}_{4}$ which is neither irreducible nor hyperelliptic is adjacent to $\mathcal{Q}^{\text {irr,I }}(12)$ or $\mathcal{Q}^{\text {irr,II }}(12)$ (Theorem 7.1). In order to prove the theorem it suffices to show that those 
components are in fact adjacent to $\mathcal{Q}^{\text {irr,I }}(12)$. By Theorem 7.2, any non-irreducible or nonhyperelliptic component is adjacent to a non-irreducible or non-hyperelliptic component of a stratum of $\mathcal{Q}_{4}$ with $n=2$. Let $\mathcal{C}$ be any non-irreducible or non-hyperelliptic component of $\mathcal{Q}\left(k_{1}, k_{2}\right)$ with $k_{1}+k_{2}=12$. One has to prove that $\mathcal{C}$ is adjacent to $\mathcal{Q}^{\mathrm{irr}, \mathrm{I}}(12)$. By Theorem 7.1, $\mathcal{C}$ is adjacent to $\mathcal{Q}^{\text {irr,I }}(12)$ or $\mathcal{Q}^{\text {irr,II }}(12)$. Let us assume that the second case holds. By Proposition B.3 there exists $\mathcal{C}^{\prime} \subset \mathcal{Q}\left(k_{1}, k_{2}\right)$ such that $\mathcal{Q}^{\text {irr,II }}(12) \subset \overline{\mathcal{C}^{\prime}}$ and $\mathcal{Q}^{\text {irr,I }}(12) \subset \overline{\mathcal{C}^{\prime}}$. Therefore $\mathcal{Q}^{\text {irr,II }}(12) \subset \overline{\mathcal{C}^{\prime}} \cup \overline{\mathcal{C}}$ and Corollary 2.7 implies $\mathcal{C}=\mathcal{C}^{\prime}$. Theorem 1.2 is proven.

\section{Appendix A}

\section{Connectedness of particular strata}

This section is devoted to the computation of the connected components for strata in low dimension.

Lemma A.1. - The stratum $\mathcal{Q}(8)$ is connected.

Proof. - Let $(\mathcal{S}(\pi, \lambda), q)$ be a genus three flat surface, with a single singularity. The conical angle around this singularity is $10 \pi$. We will show that the number of possibilities for the combinatorics of the gluing maps of the set of horizontal separatrix loops is very small.

Recall that with each permutation $\pi$ one associates two lines of the table representing $\pi$ (see Section 3). There are obviously two possibilities for these two lines: either the numbers of elements are equal or they are different. One defines $\mathcal{A}_{1}$ to be the set of generalized permutations corresponding to the first case. We also define $\mathcal{A}_{2}$ to be the set of generalized permutations corresponding to the second case.

By assumption $(\mathcal{S}, q) \in \mathcal{Q}(8)$, hence there are exactly five separatrix loops. Therefore the number of elements of the lines for permutations in $\mathcal{A}_{1}$ is $(5,5)$ and for permutations in $\mathcal{A}_{2}$ is $(6,4)$. A straightforward computation shows that the two sets $\mathcal{A}_{1}, \mathcal{A}_{2}$ are very simple. More precisely, up to cyclic order, one has $\# \mathcal{A}_{1}=4$ and $\# \mathcal{A}_{2}=3$.

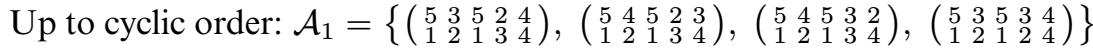

$$
\begin{aligned}
& \text { Up to cyclic order: } \mathcal{A}_{2}=\left\{\left(\begin{array}{llllll}
5 & 2 & 5 & 3 & 4 & 2 \\
1 & 3 & 1 & 4 & 4
\end{array}\right),\left(\begin{array}{llllll}
3 & 5 & 4 & 2 & 5 & 2 \\
1 & 3 & 1 & 4 & 4
\end{array}\right),\left(\begin{array}{llllll}
5 & 3 & 2 & 5 & 4 & 2 \\
1 & 3 & 1 & 4
\end{array}\right)\right\}
\end{aligned}
$$

This proves that the stratum $\mathcal{Q}(8)$ has at most 7 connected components. Now, let us consider surfaces $\mathcal{S}\left(\pi, \lambda_{2}\right)$ with $\pi \in \mathcal{A}_{2}$ and $\lambda_{2}=(1,1,1,1,1,1,2,1,2,1)$. A direct verification shows that the vertical foliation on surfaces $\mathcal{S}=\mathcal{S}\left(\pi, \lambda_{2}\right)$ decomposes $\mathcal{S}$ in a single cylinder. Therefore, we get a permutation encoding this cylinder. One can check that this permutation belongs to the set $\mathcal{A}_{1}$. This procedure connects permutations of $\mathcal{A}_{2}$ to permutations of $\mathcal{A}_{1}$.

To conclude, let us consider surfaces $\mathcal{S}\left(\pi, \lambda_{1}\right)$ with $\pi \in \mathcal{A}_{1}$ and $\lambda_{1}=(1,1,1,1,1,1,1,1,1,1)$. These surfaces are arithmetic surfaces. It is easy to check that all of these surfaces belong to the same $\mathrm{PSL}_{2}(\mathbb{Z})$-orbit. The lemma is proven.

Lemma A.2. - The stratum $\mathcal{Q}(12)$ has at most two connected components.

$4^{\text {e }}$ SÉRIE - TOME $41-2008-\mathrm{N}^{\mathrm{o}} 1$ 
Proof. - One has to show that $\mathcal{Q}(12)=\mathcal{Q}^{\text {irr,I }}(12) \cup \mathcal{Q}^{\text {irr,II }}(12)$. Thanks to the previous lemma, let $\mathcal{C}_{0}$ be the unique connected component of the stratum $\mathcal{Q}(8)$. Theorem 6.3 implies that any component $\mathcal{C}$ of the stratum $\mathcal{Q}(12)$ has the following form

$$
\mathcal{C}=\mathcal{C}_{0} \oplus s \text { with } s=1, \ldots, 6=2 g .
$$

We now recall the construction of the two irreducible components $\mathcal{Q}^{\text {irr,I }}(12), \mathcal{Q}^{\text {irr,II }}(12)$.

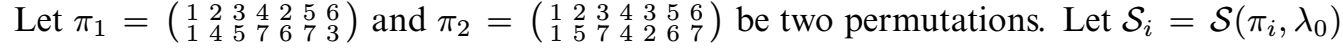
be the suspended flat surfaces with admissible vector $\lambda_{0}=(1,1,1,1,1,1,1)$. By definition $\mathcal{Q}^{\text {irr,I }}(12)$ is the component containing $\mathcal{S}_{1}$ and $\mathcal{Q}^{\text {irr,II }}(12)$ is the component containing $\mathcal{S}_{2}$. Let us recall (see Lemma 5.14) that the vertical foliation on $\mathcal{S}_{i}$ produces a multiplicity one simple cylinder which gives:

$$
\begin{aligned}
& \mathcal{Q}^{\text {irr,I }}(12)=\mathcal{C}_{0} \oplus 2 \\
& \mathcal{Q}^{\text {irr,II }}(12)=\mathcal{C}_{0} \oplus 6 .
\end{aligned}
$$

We will show:

$$
\begin{aligned}
& \mathcal{C}_{0} \oplus 4=\mathcal{C}_{0} \oplus 1=\mathcal{C}_{0} \oplus 5=\mathcal{C}_{0} \oplus 2=\mathcal{Q}^{\text {irr }, \mathrm{I}}(12) \\
& \mathcal{C}_{0} \oplus 3=\mathcal{C}_{0} \oplus 6=\mathcal{Q}^{\text {irr }, \mathrm{II}}(12) .
\end{aligned}
$$

Combining with above Equation (A.1) this will give the lemma.

Let $\mathcal{C}_{0} \oplus s, s=1, \ldots, 6$ be any component of $\mathcal{Q}(12)$.

Case $s=3$.

Let us consider the new permutation $\pi_{2} \sim \pi_{2}^{\prime}=\left(\begin{array}{ccccccc}5 & 6 & 1 & 2 & 3 & 4 & 3 \\ 5 & 7 & 4 & 2 & 6 & 7 & 1\end{array}\right)$. Obviously, $\hat{\pi}_{2}^{\prime}$ is irreducible; thus one gets a (vertical) multiplicity one simple cylinder on $\mathcal{S}\left(\pi_{2}^{\prime}, \lambda_{0}\right)$. This cylinder has angle $3 \pi$. In other words $\mathcal{C}_{0} \oplus 3=\mathcal{Q}^{\text {irr,II }}(12)$.

Case $s=4$.

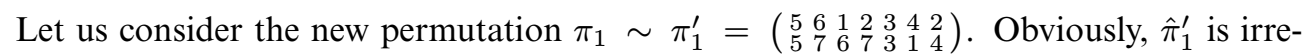
ducible; thus one gets a (vertical) multiplicity one simple cylinder on $\mathcal{S}\left(\pi_{1}^{\prime}, \lambda_{0}\right)$. This cylinder has angle $4 \pi$ or in other words $\mathcal{C}_{0} \oplus 4=\mathcal{Q}^{\text {irr,I }}(12)$.

Cases $s=1$ and $s=5$.

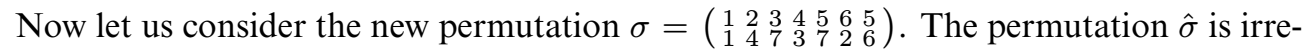
ducible; therefore the surface $\mathcal{S}\left(\sigma, \lambda_{0}\right)$ has a (vertical) multiplicity one simple cylinder. This cylinder has angle $4 \pi$, therefore this surface $\mathcal{S}\left(\sigma, \lambda_{0}\right)$ belongs to component $\mathcal{Q}^{\text {irr,I }}(12)$.

Let us consider the new permutation $\sigma \sim \sigma^{\prime}=\left(\begin{array}{ccccccc}3 & 4 & 5 & 6 & 5 & 1 & 2 \\ 3 & 7 & 2 & 6 & 1 & 4 & 7\end{array}\right)$. Obviously, $\hat{\sigma}^{\prime}$ is irreducible; thus one gets a (vertical) multiplicity one simple cylinder on $\mathcal{S}\left(\sigma^{\prime}, \lambda_{0}\right)$. This cylinder has angle $\pi$. In other words $\mathcal{C}_{0} \oplus 1=\mathcal{Q}^{\text {irr,I }}(12)$.

Let us consider the new permutation $\sigma \sim \sigma^{\prime \prime}=\left(\begin{array}{lllllll}2 & 3 & 4 & 5 & 6 & 5 & 1 \\ 2 & 6 & 1 & 4 & 7 & 7 & 7\end{array}\right)$. Obviously, $\hat{\sigma}^{\prime \prime}$ is irreducible; thus one gets a (vertical) multiplicity one simple cylinder on $\mathcal{S}\left(\sigma^{\prime \prime}, \lambda_{0}\right)$. This cylinder has angle $5 \pi$ or in other words $\mathcal{C}_{0} \oplus 5=\mathcal{Q}^{\text {irr,I }}(12)$.

The lemma is proven.

Lemma A.3. - The stratum $\mathcal{Q}(-1,5)$ is connected. 
Proof of Lemma A.3. - Let $\mathcal{S}(\pi, \lambda) \in \mathcal{Q}(-1,5)$ be any surface. One can directly check that (up to cyclic order) there are only two combinatorics for the permutation $\pi$

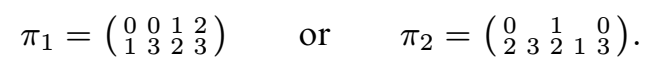

Equipped with the two admissible vectors

$$
\lambda_{1}=(1,1,2,1,2,1,1,1) \quad \text { and } \quad \lambda_{2}=(2,1,2,1,1,1,1,1),
$$

we obtain two flat surfaces $\mathcal{S}_{i}=\mathcal{S}\left(\pi_{i}, \lambda_{i}\right), i=1,2$. A direct computation shows that the vertical foliation on $\mathcal{S}_{2}$ produces the surface $\mathcal{S}_{1}$. The lemma is proven.

Proposition A.4. - The following assertions hold:

1. For any $s \in\{1,2,4\}$, there exists a flat surface $(\mathcal{S}, q) \in \mathcal{Q}(-1,5) \oplus s$ with a multiplicity one saddle connection.

2. Any connected component of $\mathcal{Q}(-1,13)$ has a flat surface with a multiplicity one saddle connection.

3. If $(\mathcal{S}, q)$ has a multiplicity one saddle connection, then $(\mathcal{S}, q) \oplus s$ has also a multiplicity one saddle connection by bubbling a handle.

Proof of Proposition A.4. - We consider separately the three cases.

\section{Proof of the first point}

We will present three surfaces in $\mathcal{Q}(-1,5) \oplus s$ (for $s=1,2,4)$ with a multiplicity one saddle connection. For that we construct surfaces in $\mathcal{Q}(-1,9)$ with a multiplicity one simple cylinder of angle $\pi, 2 \pi$ and $4 \pi$.

Thus let $\pi_{1}=\left(\begin{array}{llllll}3 & 4 & 0 & 0 & 1 & 2 \\ 3 & 5 & 2 & 1 & 4 & 5\end{array}\right)$ and $\pi_{2}=\left(\begin{array}{llllll}2 & 3 & 4 & 0 & 0 & 1 \\ 2 & 4 & 5 & 1 & 3 & 5\end{array}\right)$ be two permutations. The corresponding surfaces belong to $\mathcal{Q}(-1,9)$. The surfaces $\mathcal{S}_{1}=\mathcal{S}\left(\pi_{1}, \lambda\right)$ and $\mathcal{S}_{2}=\mathcal{S}\left(\pi_{2}, \lambda\right)$ possess a (vertical) multiplicity one simple cylinder. One can check that $\hat{\pi}_{i}$ is irreducible for $i=1,2$; thus, by Theorem 5.13, each of the surfaces $\mathcal{S}_{1}$ and $\mathcal{S}_{2}$ possesses a multiplicity one simple cylinder. Thanks to a direct computation, this cylinder has angle $\pi$ for $i=1$ and $2 \pi$ for $i=2$. In other words this proves $\mathcal{S}_{1} \in \mathcal{Q}(-1,5) \oplus 1$ and $\mathcal{S}_{2} \in \mathcal{Q}(-1,5) \oplus 2$.

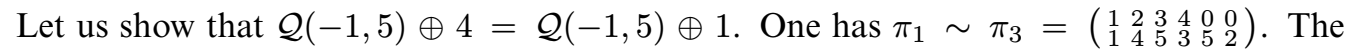
permutation $\hat{\pi}_{3}$ is irreducible and the angle of the cylinder on $\mathcal{S}\left(\pi_{3}, \lambda\right)$ is $4 \pi$. Then the surface $\mathcal{S}\left(\pi_{3}, \lambda\right)$ belongs to $\mathcal{Q}(-1,5) \oplus 4$ and $\mathcal{Q}(-1,5) \oplus 1$.

To end the proof it remains to find a multiplicity one saddle connection on each surface $\mathcal{S}_{1}, \mathcal{S}_{2}$. This is done with the following remark. Let us consider the permutations

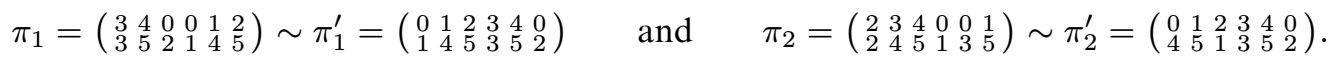

For $i=1,2$ the vertical separatrix loop $\gamma\left(\pi_{i}^{\prime}\right)$ is a saddle connection and $\pi_{i}^{\prime}$ is irreducible. Therefore Proposition 5.10 applies. Hence the first point of the proposition is proven.

$4^{\mathrm{e}}$ SÉRIE - TOME $41-2008-\mathrm{N}^{\mathrm{o}} 1$ 


\section{Proof of the second point}

One has to prove that each component of $\mathcal{Q}(-1,13)$ has a flat surface with a multiplicity one saddle connection. Thanks to Proposition 7.8, we are reduced to consider components of the form $\mathcal{C} \oplus s$ with $\mathcal{C} \subset \mathcal{Q}(-1,9)$. The previous proof shows that each component of $\mathcal{Q}(-1,9)$ (different from $\mathcal{Q}^{\text {irr }}(-1,9)$ ) possesses a surface with a multiplicity one saddle connection. Finally one has to consider components of the form

$$
\mathcal{Q}^{\text {irr }}(-1,9) \oplus s \quad \text { with } s=1, \ldots, 6
$$

Let us recall that $\mathcal{Q}^{\text {irr }}(-1,9)=\mathcal{Q}(-1,5) \oplus 3$. Moreover any component $\mathcal{Q}(-1,5) \oplus s$, with $s=1,2,4,5$, possesses a flat surface with a multiplicity one saddle connection. Using properties of the map $\oplus$ (see Proposition 2.9), this yields to $\mathcal{Q}(-1,9)^{\mathrm{irr}} \oplus s=\mathcal{Q}(-1,5) \oplus s \oplus 3$ which proves the proposition for $s=1,2,4,5$.

The case $s=6$ is reduced to the case $s=3$.

$$
\mathcal{Q}(-1,9)^{\text {irr }} \oplus 6=\mathcal{Q}(-1,5) \oplus 6 \oplus 3=\mathcal{Q}(-1,9)^{\text {irr }} \oplus 3 .
$$

We finish the proof of the second statement using the second property of the map $\oplus$.

$$
\mathcal{Q}(-1,5) \oplus 3 \oplus 3=\mathcal{Q}(-1,5) \oplus 1 \oplus 5
$$

The proof of the third part is obvious and left to the reader.

We finish this section with an independent proof of a theorem of Masur and Smillie [15]. The original proof uses algebraic geometry. Here we only use combinatorics of generalized permutations.

Theorem (Masur, Smillie). - The following strata

$$
\mathcal{Q}(\varnothing), \mathcal{Q}(1,-1)(\text { in genus } g=1) \text { and } \mathcal{Q}(4), \mathcal{Q}(1,3) \text { (in genus } g=2 \text { ) }
$$

are empty.

Proof of the theorem. - Let us assume that the stratum $\mathcal{Q}(4)$ is non-empty. Thus there exists a genus two flat surface with a single zero. Applying "breaking up a singularity" to this zero in two zeroes of order two we get a point inside the stratum $\mathcal{Q}(2,2)$ (see [11] or [17], [3]). By construction, this new surface has a multiplicity one saddle connection so it belongs to a non-hyperelliptic component of $\mathcal{Q}(2,2)$. Now we will prove that this stratum is connected and equal to its hyperelliptic component which leads to a contradiction.

Let us consider a point $\mathcal{S}(\pi, \lambda) \in \mathcal{Q}(2,2)$. As usual, a direct computation shows that one can put (up to cyclic order) $\pi$ in one of the two following forms $\left(\begin{array}{llll}1 & 2 & 1 & 3 \\ 4 & 3 & 4 & 2\end{array}\right)$ or $\left(\begin{array}{llll}1 & 2 & 1 & 2 \\ 3 & 4 & 3 & 4\end{array}\right)$. According to Lemma 4.2, each of these two permutations gives rise to surfaces in the component $\mathcal{Q}^{\text {hyp }}(2,2)$. Therefore $\mathcal{Q}(2,2)=\mathcal{Q}^{\text {hyp }}(2,2)$ is connected and hence the stratum $\mathcal{Q}(4)=\varnothing$.

Using the same approach, we prove that $\mathcal{Q}(1,-1)$ and $\mathcal{Q}(1,3)$ are empty (one considers respectively the hyperelliptic connected components $\mathcal{Q}(-1,-1,2)$ and $\mathcal{Q}(1,1,2)$ ).

The stratum $\mathcal{Q}(\varnothing)$ is empty because the quotient of a holomorphic quadratic differential $q$ by $d z^{2}$ is a holomorphic function on the torus thus constant. Therefore $q=\omega^{2}$ which is a contradiction. 


\section{Appendix B}

\section{Deformations of hyperelliptic and irreducible components}

Proposition B.1. - Let $(\mathcal{S}, q) \in \mathcal{Q}^{\text {hyp }}(4(g-k)-6,4 k+2)$ be a point with $0 \geq k \geq g-2$ and $g \geq 2$. Let $\left(k_{1}, k_{2}\right)$ be a positive partition of $4 k+2$. Then there exists a continuous path $\rho:[0,1] \longrightarrow \mathcal{Q}_{g}$ of the interval $[0,1]$ in the whole moduli space $\mathcal{Q}_{g}$ such that:

- $\rho(0)=(\mathcal{S}, q)$

- $\rho(t) \in \mathcal{Q}\left(4(g-k)-6, k_{1}, k_{2}\right) \quad \forall 0<t<1$.

- $\rho(1) \in \mathcal{Q}(4(g-k)-6,4 k+2) \backslash \mathcal{Q}^{\text {hyp }}(4(g-k)-6,4 k+2)$.

Proof. - We remark that it is sufficient to prove the proposition for a particular point of the component $\mathcal{Q}^{\text {hyp }}(4(g-k)-6,4 k+2)$. We first claim:

Claim. - Let us fix $r=2 k+1$ and $l=2(g-k)-3$. Let a be any integer with $2 \leq a \leq r+1$. Let us consider the generalized permutation

$$
\Pi_{1}(r, l, a)=\left(\begin{array}{ccccccccc}
0_{1} & 0_{3} & 1 & \ldots & r & 0_{1} & r+1 \ldots & r+l \\
r+l \ldots & r+1 & 0_{2} & r \ldots & \ldots & 0_{3} & a-1 \ldots & 1 & 0_{2}
\end{array}\right) .
$$

Then for any admissible vector $\lambda$ one has $\mathcal{S}\left(\Pi_{1}(r, l, a), \lambda\right) \in \mathcal{Q}(a-2,4 k+4-a, 4(g-k)-$ $3)$. Moreover, the horizontal saddle connection labeled $0_{3}$ has multiplicity one. The resulting surface $\tilde{S}$ obtained by shrinking this saddle connection to a point is $\mathcal{S}\left(\Pi_{1}(r, l), \lambda\right)$ that belongs to the hyperelliptic component.

Proof of the claim. - A straightforward calculation of the angle of the conical singularities located at end-points of the intervals gives the result.

The proposition follows from above claim taking $a=k_{1}+2$.

One can easily have similar results on deformations of hyperelliptic components of other strata. Here we present a similar result concerning irreducible components. The proof is just based on the deformations of an adequate generalized permutation.

Proposition B.2. - Let $(\mathcal{S}, q) \in \mathcal{Q}^{\mathrm{irr}}(-1,9)$ be a point. Let $\left(k_{1}, k_{2}\right)$ be any pair in the list $\{(-1,10),(1,8),(2,7),(3,6),(4,5)\}$. Then there exists a continuous path $\rho:[0,1] \longrightarrow \mathcal{Q}_{3}$ of the interval $[0,1]$ in the whole moduli space $\mathcal{Q}_{3}$ such that:

- $\rho(0)=(\mathcal{S}, q)$

- $\rho(t) \in \mathcal{Q}\left(-1, k_{1}, k_{2}\right) \quad \forall 0<t<1$.

- $\rho(1) \in \mathcal{Q}(-1,9) \backslash \mathcal{Q}^{\text {irr }}(-1,9)$.

Proof. - The proof is similar to the previous one, deforming the permutation $\left(\begin{array}{lllllll}0 & 1 & 2 & 3 & 4 & 4 & 0 \\ 4 & 3 & 2 & 5 & 1 & 5\end{array}\right)$ representing the irreducible component of $\mathcal{Q}(-1,9)$.

Proposition B.3. - Let $(\mathcal{S}, q) \in \mathcal{Q}^{\text {irr,II }}(12)$ be a point. Let $\left(k_{1}, k_{2}\right)$ be any pair in the list $\{(-1,13),(1,11),(2,10),(3,9),(4,8),(5,7),(6,6)\}$.

Then there exists a continuous path $\rho:[0,1] \longrightarrow \mathcal{Q}_{4}$ of the interval $[0,1]$ in the whole moduli space $\mathcal{Q}_{4}$ such that:

- $\rho(0)=(\mathcal{S}, q)$

- $\rho(t) \in \mathcal{Q}\left(k_{1}, k_{2}\right) \quad \forall 0<t<1$.

$4^{\text {e }}$ SÉRIE - TOME $41-2008-\mathrm{N}^{\mathrm{o}} 1$ 
- $\rho(1) \in \mathcal{Q}^{\text {irr,I }}(12)$

Proof. - The proof parallels the one of Proposition B.1 deforming the permutation $\left(\begin{array}{lllllll}1 & 2 & 3 & 4 & 3 & 5 & 6 \\ 1 & 5 & 7 & 4 & 2 & 6 & 7\end{array}\right)$.

\section{REFERENCES}

[1] C. Boissy, E. Lanneau, Dynamics and geometry of the Rauzy-Veech induction for quadratic differentials, preprint arXiv:0710.5614, 2007.

[2] A. Doundy, J. Hubbard, On the density of Strebel differentials, Invent. Math. 30 (1975), 175-179.

[3] A. Eskin, H. Masur, A. Zorich, Moduli spaces of abelian differentials: the principal boundary, counting problems, and the Siegel-Veech constants, Publ. Math. Inst. Hautes Études Sci. 97 (2003), 61-179.

[4] A. Fathi, H. Masur, V. Poenaru, Travaux de Thurston sur les surfaces, Astérisque 66, Soc. Math. France, 1979.

[5] J. Hubbard, H. Masur, Quadratic differentials and foliations, Acta Math. 142 (1979), 221-274.

[6] M. Keane, Interval exchange transformations, Math. Z. 141 (1975), 25-31.

[7] S. Kerckhoff, H. Masur, J. Smillie, Ergodicity of billiard flows and quadratic differentials, Ann. of Math. 124 (1986), 293-311.

[8] M. Kontsevich, A. Zorich, Connected components of the moduli spaces of Abelian differentials with prescribed singularities, Invent. Math. 153 (2003), 631-678.

[9] M. Kontsevich, A. Zorich, Lyapunov exponents and Hodge theory, preprint arXiv:hep-th/9701164v1, 2007.

[10] E. LanneaU, Classification of connected components of the strata of the moduli spaces of quadratic differentials with prescribed singularities, Ph.D. Thesis, Universite de Rennes 1, december 2003.

[11] E. Lanneau, Hyperelliptic components of the moduli spaces of quadratic differentials with prescribed singularities, Comment. Math. Helv. 79 (2004), 471-501.

[12] E. Lanneau, Parity of the spin structure defined by a quadratic differential, Geom. Topol. 8 (2004), 511-538.

[13] H. Masur, The Jenkins-Strebel differentials with one cylinder are dense, Comment. Math. Helv. 54 (1979), 179-184.

[14] H. Masur, Interval exchange transformations and measured foliations, Ann. of Math. 115 (1982), 169-200. 
[15] H. Masur, J. Smillie, Quadratic differentials with prescribed singularities and pseudo-Anosov diffeomorphisms, Comment. Math. Helv. 68 (1993), 289-307.

[16] H. Masur, S. Tabachnikov, Rational billiards and flat structures, in Handbook of dynamical systems, Vol. 1A, North-Holland, 2002, 1015-1089.

[17] H. Masur, A. Zorich, Moduli spaces of quadratic differentials: The principal boundary, counting problems and the Siegel-Veech constants, $G A F A$ to appear.

[18] G. Rauzy, Échanges d'intervalles et transformations induites, Acta Arith. 34 (1979), 315-328.

[19] K. Strebel, Quadratic differentials, Ergebnisse der Mathematik und ihrer Grenzgebiete (3) 5, Springer, 1984.

[20] W. P. Thurston, On the geometry and dynamics of diffeomorphisms of surfaces, Bull. Amer. Math. Soc. (N.S.) 19 (1988), 417-431.

[21] W. A. Veech, Gauss measures for transformations on the space of interval exchange maps, Ann. of Math. 115 (1982), 201-242.

[22] W. A. Veech, The Teichmüller geodesic flow, Ann. of Math. 124 (1986), 441-530.

[23] W. A. VeEch, Moduli spaces of quadratic differentials, J. Analyse Math. 55 (1990), 117 171.

[24] A. Zorich, Flat surfaces, in Frontiers in number theory, physics, and geometry. I, Springer, 2006, 437-583.

[25] A. Zorich, Explicit Jenkins-Strebel representatives of all strata of Abelian and quadratic differentials, Journal of Modern Dynamics, to appear.

[26] A. Zorich, Rauzy-Veech induction, Rauzy classes, generalized permutations on Mathematica, http://perso.univ-rennes1.fr/anton.zorich.

\footnotetext{
Erwan LANNEAU

Centre de Physique Théorique de Marseille (CPT), UMR CNRS 6207

Université du Sud Toulon-Var et

Fédération de Recherches des Unités de

Mathématiques de Marseille Luminy

Case 907

F-13288 Marseille Cedex 9, France

E-mail: lanneau@cpt.univ-mrs.fr
} 